LEANDRO FERNANDES BERGAMO

\title{
Instabilidade hidrodinâmica linear do escoamento compressível em uma cavidade
}





\section{LEANDRO FERNANDES BERGAMO}

\section{Instabilidade hidrodinâmica linear do escoamento compressível em uma cavidade}

Dissertação apresentada à Escola de Engenharia de São Carlos, da Universidade de São Paulo, como parte dos requisitos para obtenção do título de Mestre em Engenharia Mecânica.

Área de Concentração: Aeronaves

Orientador: Prof. Dr. Marcello A. F. de Medeiros

ESTE EXEMPLAR TRATA-SE DA VER-
SÃO CORRIGIDA. A VERSÃO ORIGINAL
ENCONTRA-SE DISPONÍVEL JUNTO AO
DEPARTAMENTO DE ENGENHARIA ME-
CÂNICA DA EESC-USP.

São Carlos 
AUTORIZO A REPRODUÇÃO TOTAL OU PARCIAL DESTE TRABALHO, POR QUALQUER MEIO CONVENCIONAL OU ELETRÔNICO, PARA FINS DE ESTUDO E PESQUISA, DESDE QUE CITADA A FONTE.

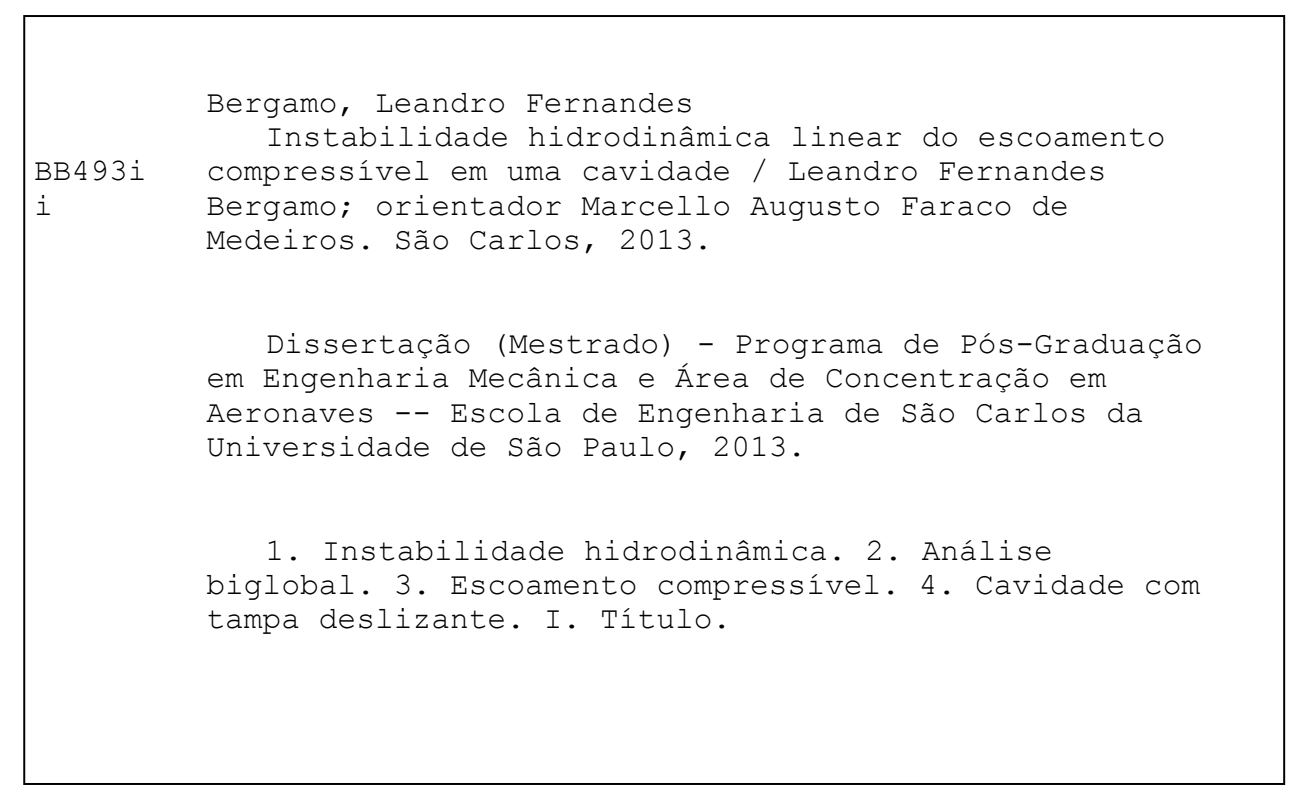




\section{FOLHA DE JULGAMENTO}

Candidato: Engenheiro LEANDRO FERNANDES BERGAMO.

Título da dissertação: "Instabilidade hidrodinâmica linear do escoamento compressível em uma cavidade".

Data da defesa: $28 / 04 / 2014$

\section{Comissão Julgadora:}

\section{Resultado:}

Prof. Associado Marcello Augusto Faraco de Medeiros (orientador)

Aprovado

(Escola de Engenharia de São Carlos/EESC)

Prof. Dr. Sávio Brochini Rodrigues

(Universidade Federal de São Carlos/UFSCar)

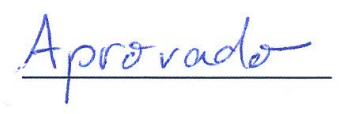

Prof. Dr. André Valdetaro Gomes Cavalieri

(Instituto Tecnológico de Aeronáutica/ITA)

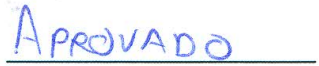

Coordenador do Programa de Pós-Graduação em Engenharia Mecânica:

Prof. Associado Marcelo Areias Trindade

Presidente da Comissão de Pós-Graduação:

Prof. Titular Denis Vinicius Coury 

À minha família. 



\section{Agradecimentos}

Ao meu orientador, Prof. Dr. Marcello Augusto Faraco de Medeiros, pelos ensinamentos, incentivo e orientação no desenvolvimento desta dissertação.

Ao amigo Dr. Elmer Mateus Gennaro, por ter sido, efetivamente, um co-orientador neste projeto.

Aos pesquisadores Dr. Daniel Rodríguez e Prof. Dr. Vassilis Theofilis, pela ajuda necessária à realização deste trabalho.

À minha família, pelo carinho e apoio.

Aos amigos das salas de pós-graduação, pela companhia e discussões durante o café.

Aos amigos da Kit Isabel, pela grande amizade e pelos memoráveis momentos.

À Escola de Engenharia de São Carlos, pela infraestrutura concedida.

Ao Conselho Nacional de Desenvolvimento Científico e Tecnológico (CNPq), pelo apoio financeiro.

A todos que de alguma maneira contribuíram para esta dissertação. 


\section{Sumário}

Lista de Figuras

Lista de Tabelas

Resumo

Abstract

xxiii

1 Introdução

2 Fundamentos Teóricos

2.1 Equações Governantes . . . . . . . . . . . . . . . . . . . . . 31

2.2 Instabilidade Hidrodinâmica Linear ～. . . . . . . . . . . . . . . . . . 32

2.2.1 Teoria Clássica . . . . . . . . . . . . . . . . . . 34

2.2.2 Análise Temporal e Espacial . . . . . . . . . . . . . . . . . . 35

2.2.3 Instabilidade Convectiva e Absoluta . . . . . . . . . . . . . . . 35

2.2 .4 Análise Biglobal . . . . . . . . . . . . . . . . 36

2.2.5 Não Normalidade do Operador Linear . . . . . . . . . . . . . . . . . . 37 


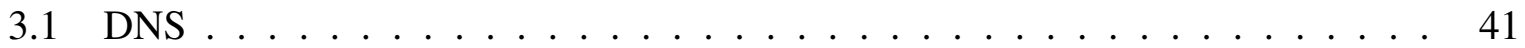

3.1.1 Discretização Espacial . . . . . . . . . . . . . . . . . . . 42

3.1 .2 Condições de Contorno . . . . . . . . . . . . . . . . . . . . . 48

3.1 .3 Integração Temporal . . . . . . . . . . . . . . . . . . . 51

3.1 .4 Métrica ......................... 52

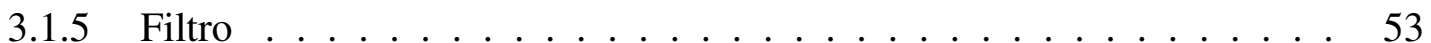

3.1.6 Zona de Amortecimento . . . . . . . . . . . . . . . . 55

3.1 .7 Paralelização . . . . . . . . . . . . . . . . . 58

3.2 Instabilidade Hidrodinâmica Linear . . . . . . . . . . . . . . . . . 60

3.2.1 Discretização Espacial . . . . . . . . . . . . . . . . . . 60

3.2 .2 Condições de Contorno . . . . . . . . . . . . . . . . . 63

3.2.3 Problema de Autovalor . . . . . . . . . . . . . . . . . . . . 64

3.2 .4 Pseudo Espectro . . . . . . . . . . . . . . . . . 66

4 Resultados $\quad 69$

4.1 Equação de Helmholtz ～. . . . . . . . . . . . . . . . . . . . . . . . . . . 69

4.2 Escoamento Incompressível em um Duto . . . . . . . . . . . . . . . 71

4.3 Cavidade Compressível . . . . . . . . . . . . . . . . . . . . 77

4.3.1 Escoamento Base . . . . . . . . . . . . . . . . . . 77

4.3.2 Instabilidade Linear . . . . . . . . . . . . . . . . . . . 81

Comparação com o Escoamento Incompressível . . . . . . . . . . 84

Efeito do Número de Mach . . . . . . . . . . . . . . . . . . . 86 
Modos Acústicos de Duto . . . . . . . . . . . . . . . . . . . . . 90

Modos Acústicos de Cavidade . . . . . . . . . . . . . . . . . . . 95

Pseudo Espectro . . . . . . . . . . . . . . . . . . . . . 98

5 Conclusões $\quad 101$

Referências Bibliográficas

APÊNDICE A - Operador linear incompressível

APÊNDICE B - Operador linear compressível 


\section{Lista de Figuras}

Figura - 1.1 Ilustração do experimento de Reynolds.

Figura - 1.2 (AIDUN; TRIANTAFILLOPOULOS; BENSON, 1991). (a) estado estacionário, $R e \simeq 500$; (b) estado periódico, $R e \simeq 900$; (c) oscilações no vórtice secundário, $R e \simeq 1200$; (d) estruturas similares a vórtices de Taylor-Görtler, $\operatorname{Re}>1900$.

Figura - 1.3 (AIDUN; TRIANTAFILLOPOULOS; BENSON, 1991). Estados estacionários. (a) duas células, (b) três células, (c) quatro células.

Figura - 2.1 Instabilidade convectiva (a) e absoluta (b).

Figura - 2.2 (SCHMID, 2007). Crescimento transiente observado entre dois modos $\Phi_{1}$ e $\Phi_{2}$. O vetor $f$ é a diferença entre os dois modos.

Figura - 3.1 Número de onda modificado para diferenças finitas compactas com alta resolução espectral utilizando as restrições das tabelas 3.1 e 3.2 .

Figura - 3.2 Velocidade de fase para diferenças finitas compactas com alta resolução espectral utilizando as restrições das tabelas 3.1 e 3.2 .

Figura - 3.3 Ordem de precisão para diferenças finitas compactas com alta resolução espectral utilizando as restrições das tabelas 3.1 e 3.2.

Figura - 3.4 Autovalores da equação de convecção discretizada. 
Figura - 3.5 Comparação do método proposto com as diferenças finitas obtidas por Lele (1992).

Figura - 3.6 Função de resposta em frequência do filtro compacto.

Figura - 3.7 Geometria da expansão abrupta.

Figura - 3.8 Perfil de velocidade $u$ na entrada da expansão abrupta.

Figura - 3.9 Contornos de $v$ para uma expansão abrupta a $R e=600$ e $M_{\infty}=0,2 . \quad \ldots \quad 57$

Figura - 3.10 Decomposição do domínio. Cada cor representa um processo.

Figura - 3.11 Escalabilidade do DNS.

Figura - 3.12 Ilustração dos estêncis das diferenças finitas. (HERMANNS, 2008).

Figura - 4.1 Convergência de malha para o autovalor $\lambda^{2}=91,29384071007$ da equação de Helmholtz. Discretização por diferenças finitas de ordens 4 (DF4), $8(D F 8)$ e 12 (DF12) e método pseudo espectral de Chebyshev-GaussLobatto $(C G L)$.

Figura - 4.2 Erro para o primeiro autovalor da equação de Helmholtz para diferentes números de vetores de Ritz $n_{e v}$ com o método de Arnoldi. Diferenças finitas de ordem 12. Malha de 30 pontos.

Figura - 4.3 Custo computacional para o autovalor dominante do duto. $\operatorname{Re}=100, \beta=1$. Discretização por CGL, DF4, DF8 e DF12.

Figura - 4.4 Convergência de malha a parte imaginária do autovalor dominante do escoamento incompressível no interior de um duto quadrado. $\operatorname{Re}=100, \beta=1$. 
Figura - 4.5 Espectro para o escoamento incompressível no interior de um duto quadrado. $\operatorname{Re}=100, \beta=1$.

Figura - 4.6 Primeiro modo do duto (autofunção 1a). $R e=100, \beta=1$.

Figura - 4.7 Primeiro modo do duto (autofunção $1 \mathrm{~b}$ ). $R e=100, \beta=1$.

Figura -4.8 Segundo modo do duto. $R e=100, \beta=1$.

Figura -4.9 Terceiro modo do duto. $R e=100, \beta=1$. 76

Figura - 4.10 Quarto modo do duto. $\operatorname{Re}=100, \beta=1$. 76

Figura - 4.11 Geometria da cavidade com tampa deslizante.

Figura - 4.12 Condição de contorno regularizada para a tampa deslizante. 78

Figura - 4.13 Convergência de malha para a variação de densidade no ponto $x=0,25$; $y=0,25$, no interior da cavidade. $R e=1000, M_{\infty}=0,5$. 78

Figura - 4.14 Posição dos vórtices na cavidade. $R e=1000, M_{\infty}=0,5$. Contornos para $u=0-\mathrm{e} v=0-$

Figura - 4.15 Escoamento base compressível para a cavidade. $R e=1000, M_{\infty}=0,5 . \quad \ldots \quad 80$

Figura - 4.16 Derivada segunda das componentes da velocidade no escoamento base da cavidade. $R e=1000, M_{\infty}=0,5$.

Figura - 4.17 Esparsidade dos operadores lineares para a cavidade compressível discretizados com diferenças finitas de ordem 12.

Figura - 4.18 Esparsidade dos operadores lineares para a cavidade compressível discreti- 
zados pelo método pseudo espectral de Chebyshev-Gauss-Lobatto.

Figura - 4.19 Curvas neutras para os quatro modos dominantes da cavidade compressível. 87

Figura - 4.20 Modo S1 para $R e=900, M_{\infty}=0,5, \beta=15$.

Figura - 4.21 Modo T1 para $R e=900, M_{\infty}=0,5, \beta=15$. 88

Figura - 4.22 Modo T2 para $R e=900, M_{\infty}=0,5, \beta=7$.

Figura - 4.23 Modo T3 para $R e=900, M_{\infty}=0,5, \beta=15$.

Figura - 4.24 Modos compressíveis para $R e=100, M_{\infty}=0,1, \beta=1$.

Figura - 4.25 Convergência de malha para o modo compressível dominante. $R e=900$, $M_{\infty}=0,5, \beta=0$.

Figura - 4.26 Comparação dos modos compressíveis da cavidade a $R e=100, M_{\infty}=0,1$, $\beta=1$ com os modos de propagação acústica em duto.

Figura - 4.27 Parte real das autofunções do modo de propagação acústica em duto. $\omega=$ $31,907-1,154 i . R e=100, M_{\infty}=0,1, \beta=0$.

Figura - 4.28 Influência da compressibilidade nos modos de propagação acústica em duto. $\operatorname{Re}=900, \beta=1$.

Figura - 4.29 Modos compressivies para $R e=900, M_{\infty}=0,5$. Variação em $\beta$. 94

Figura - 4.30 Modos acústicos de cavidade para $R e=900, M_{\infty}=0,5, \beta=0$. 95

Figura - 4.31 Parte real das autofunções do modo acústico de cavidae $\omega=65.756-$ 3.4987i. $R e=900, M_{\infty}=0,5, \beta=0$. 96

Figura - 4.32 Variação dos autovalores dos modos acústicos de cavidade com Mach e $\beta$ 
para $R e=900$.

Figura - 4.33 $p^{\prime}$ ao longo de $x=0,947 . R e=900, M_{\infty}=0,5, \beta=0$.

Figura - 4.34 Pseudo espectro para $M_{\infty}=0,1 . \operatorname{Re}=800, \beta=15$. 98

Figura - 4.35 Pseudo espectro para $M_{\infty}=0,5 . R e=800, \beta=15$.

Figura - 4.36 Pseudo espectro para $M_{\infty}=0,9 . \operatorname{Re}=800, \beta=15$. 99 


\section{Lista de Tabelas}

Tabela - 3.1 Restrições para estêncil com cinco pontos 44

Tabela - 3.2 Restrições para estêncil com sete pontos 44

Tabela - 3.3 Coeficientes para diferenças finitas compactas descentradas de quarta ordem.

Tabela - 3.4 Coeficientes para diferenças finitas não compactas descentradas de quarta ordem.

Tabela - 3.5 Coeficientes para o filtro de décima ordem. 54

Tabela - 4.1 Quatro primeiros modos do problema de autovalor de Helmholtz. 71

Tabela - 4.2 Redução do custo computacional obtida pelo método de Arnoldi com 400 vetores de Ritz. Malha de $50 \times 50$ pontos. Diferenças finitas de ordem 8 . 73

Tabela -4.3 Autovalores menos estáveis do duto. $R e=100, \beta=1$. 74

Tabela - 4.4 Posição dos vórtices na cavidade para $R e=400, M_{\infty}=0,1$. Comparação com casos incompressíveis.

Tabela - 4.5 Convergência para o primeiro modo da cavidade compressível. $R e=1000$, $M_{\infty}=0,5, \beta=15$.

Tabela - 4.6 Comparação para a cavidade. $R e=900, M a_{\infty}=0,1, \beta=7,35$. 
Tabela - 4.7 Parâmetros críticos para a cavidade compressível. $\ldots \ldots \ldots \ldots \ldots \ldots \ldots$ 


\section{Resumo}

BERGAMO, L. F. Instabilidade hidrodinâmica linear do escoamento compressível em uma cavidade. Dissertação (Mestrado) - Escola de Engenharia de São Carlos, Universidade de São Paulo, São Carlos, 2013.

Os mecanismos de instabilidade hidrodinâmica têm um papel importante no processo da transição do escoamento de laminar para turbulento. A análise da instabilidade hidrodinâmica em uma cavidade com tampa deslizante foi realizada através da decomposição em modos globais (biglobal) para avaliar o efeito da compressibilidade neste fenômeno. O escoamento base foi obtido através de simulação numérica direta (DNS). Para tal, foi desenvolvido um código DNS compressível com discretização espacial por diferenças finitas compactas de alta resolução espectral e capacidade de processamento paralelo, com um método de decomposição de domínio que mantém a precisão das diferenças finitas compactas. O escoamento base é usado para montar o problema de autovalor oriundo das equações de Navier-Stokes linearizadas para a perturbação, discretizadas por diferenças finitas explícitas. O uso de diferenças finitas em conjunto com a implementação em matrizes esparsas reduz sensivelmente o uso de memória. Através do algoritmo de Arnoldi, a ordem do problema de autovalor é reduzida e os autovalores de interesse são recuperados. Os resultados indicam o efeito estabilizante da compressibilidade nos modos dominantes da cavidade e revelam modos inerentes ao escoamento compressível, para os quais a compressibilidade tem efeito desestabilizante. Dentre estes modos compressíveis, estão presentes modos de propagação sonora em dutos e modos relacionados à geração de som na cavidade.

Palavras-chave: Instabilidade hidrodinâmica. Análise biglobal. Escoamento compressível. Cavidade com tampa deslizante. 


\section{Abstract}

BERGAMO, L. F. Linear hidrodinamic instability of compressible lid-driven cavity flow. Dissertação (Mestrado) - Escola de Engenharia de São Carlos, Universidade de São Paulo, São Carlos, 2013.

Hydrodynamic instability mechanisms play an important role in laminar to turbulent transition. Hydrodynamic instability analysis of a lid-driven cavity flow was performed by global mode decomposition (biglobal) to evaluate compressibility effects on this phenomenon. The basic flow was calculated by direct numerical simulation (DNS). A compressible DNS code was developed with spectral-like compact finite difference spatial discretization. The code allows parallel processing with a domain decomposition method that preserves the compact finite difference accuracy. The basic flow is used to form the eigenvalue problem associated to the linear NavierStokes equations for the perturbation, which were discretized by an explicit finite difference scheme. The combination of sparse matrix techniques and finite difference discretization leads to a significant memory reduction. The order of the eigenvalue problem was reduced using the Arnoldi algorithm and the eigenvalues of interest were calculated. Results show the stabilizing effect of compressibility on the leading modes and reveal some modes intrinsic to compressible flow, for which compressibility has a destabilizing effect. Among these compressible modes, there are some related to sound propagation in ducts and to sound generation inside the cavity.

Keywords: Hydrodynamic instability. Biglobal analysis. Compressible flow. Lid-driven cavity flow. 


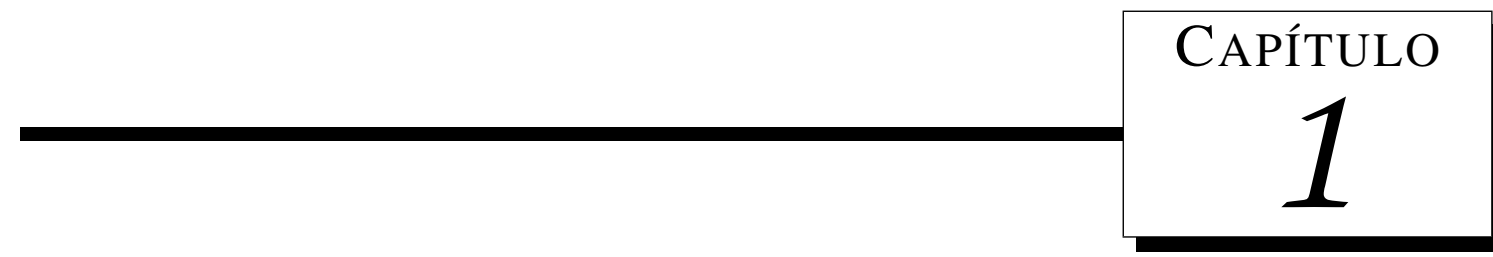

\section{Introdução}

Desde os clássicos experimentos de Reynolds (1883), que investigou o fenômeno da transição para a turbulência em escoamentos no interior de dutos (figura 1.1), a instabilidade de escoamentos laminares e a transição turbulenta tornaram-se pontos de interesse na mecânica dos fluidos, pois a transição tem impacto em grandezas hidrodinâmicas importantes, como arrasto e transferência de calor (ARNAL, 1984), além da geração de som (COLONIUS; LELE, 2004).

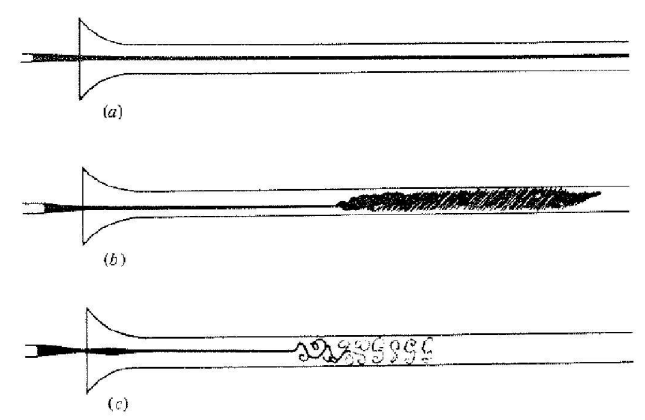

Figura 1.1: Ilustração do experimento de Reynolds.

A propagação de perturbações em uma camada limite foi estudada por Orr (1907a), Orr (1907b) e Sommerfeld (1908). Um mecanismo de amplificação de perturbações infinitesimais na equação de Orr-Sommerfeld foi teorizado pelos trabalhos de Tollmien, Schlichting e Görtler (1961) e Schlichting (1933) e demonstrado pelos experimentos de Schubauer e Skramstad (1948). 
Por muito tempo, o estudo de instabilidade hidrodinâmica linear através de análise modal restringiu-se a escoamentos base idealizados, essencialmente unidirecionais e com perturbações não homogêneas em apenas uma das direções. Tal fato se deve ao custo computacional demandado pelo problema de autovalor proveniente das equações para a perturbação quando se tem mais de uma direção não homogênea. Com a evolução do hardware disponível, análises para escoamentos base bidimensionais e tridimensionais tornaram-se possíveis.

O escoamento incompressível no interior de uma cavidade com tampa deslizante tem sido objeto de estudo da mecânica dos fluidos por muito tempo por ser um problema de geometria simples e ainda assim conter grande parte dos fenômenos possíveis em um escoamento incompressível, como vórtices, escoamento secundário, padrões tridimensionais complexos, instabilidades, transição e turbulência (SHANKAR; DESHPANDE, 2000). Essas características permitem que este escoamento seja usado para a comparação de diferentes métodos numéricos quanto a sua eficiência e precisão e também a comparação com resultados experimentais.

Um estudo numérico, através de simulação numérica direta (DNS), sobre a instabilidade hidrodinâmica da cavidade bidimensional com razão de aspecto 2 apresentado por Goodrich, Gustafson e Halasi (1990) mostra um comportamento temporal periódico assintótico para $R e=$ 5000 e $R e=10000$ e aponta a existência de uma bifurcação de Hopf entre $R e=2000$ e $R e=$ 5000.

Shen (1991), em análise feita também através de um DNS bidimensional, porém para uma cavidade com razão de aspecto 1 e condição de contorno regularizada para a velocidade da tampa, obtém soluções estacionárias até $R e=10000$. Entre $R e=10000$ e $R e=10500$ são obtidas soluções periódicas, indicando uma bifurcação de Hopf. Para $R e=15000$ a solução perde sua periodicidade, tornando-se quase periódica e indicando a existência de uma segunda bifurcação.

Os experimentos de Aidun, Triantafillopoulos e Benson (1991) mostraram que o escoamento na cavidade permanece estacionário até $R e \simeq 825$ (figura 1.2), valor muito inferior aos apresentados na literatura anteriormente. Aumentando o número de Reynolds surgem ondas no vórtice secundário próximo à base da cavidade que se movem do ponto médio do comprimento 
da cavidade em direção às paredes, dando origem a um estado periódico. Isto mostra que a instabilidade tridimensional é a que ocorre primeiro. Para $R e \simeq 1000$, a interface entre o vórtice primário e o vórtice secundário no canto inferior direito da cavidade torna-se irregular. A formação de estruturas similares a vórtices de Taylor-Görtler é observada em $R e \simeq 2000$, Indicando a existência de uma transição secundária. Outro fato interessante neste experimento foi observado ao reduzir a velocidade da tampa abruptamente de $R e=2000$ para $R e=500$. Em alguns casos o escoamento não voltava para o seu estado inicial, indicando uma instabilidade global para perturbações finitas. Foram identificados 4 estados diferentes possíveis para baixos números de Reynolds (figura 1.3).

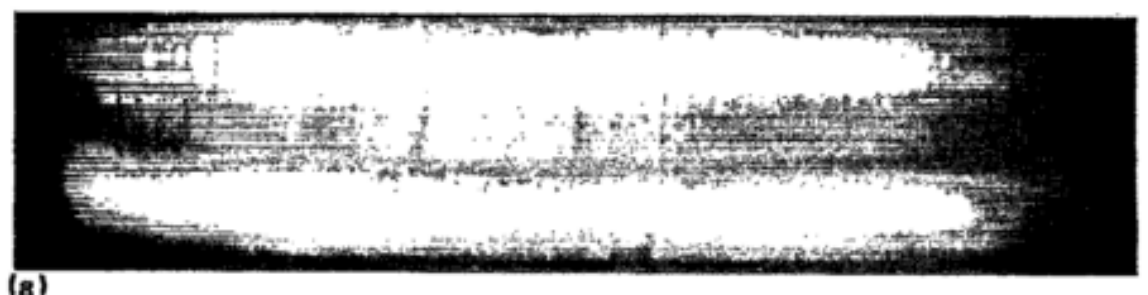

(8)

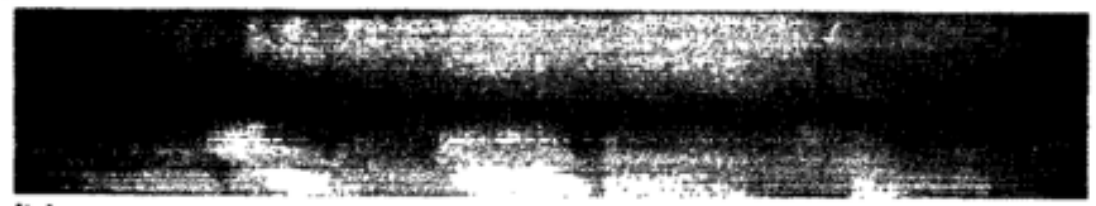

(b)

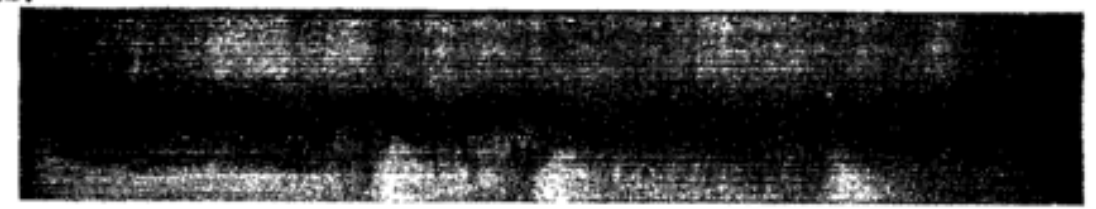

(c)

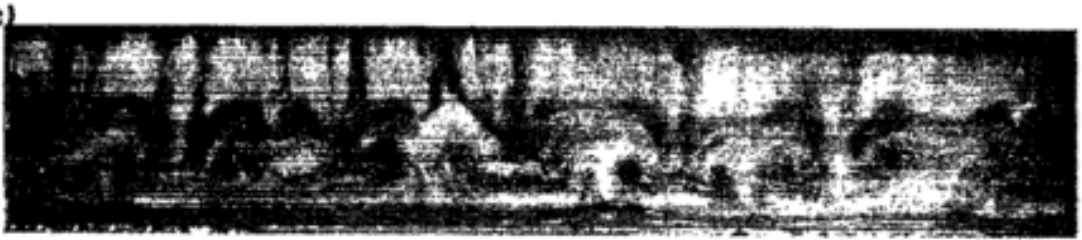

(d)

Figura 1.2: (AIDUN; TRIANTAFILLOPOULOS; BENSON, 1991). (a) estado estacionário, $R e \simeq 500$; (b) estado periódico, $R e \simeq 900$; (c) oscilações no vórtice secundário, $R e \simeq 1200$; (d) estruturas similares a vórtices de Taylor-Görtler, $R e>1900$.

Os resultados numéricos para a instabilidade, obtidos pela decomposição em modos globais, apresentados por Ding e Kawahara (1999) para números de onda $0 \leq \beta \leq 10$ não apresentavam boa concordância com os resultados experimentais. Isto é devido à existência de dois modos mais instáveis com números de onda acima da faixa calculada. 


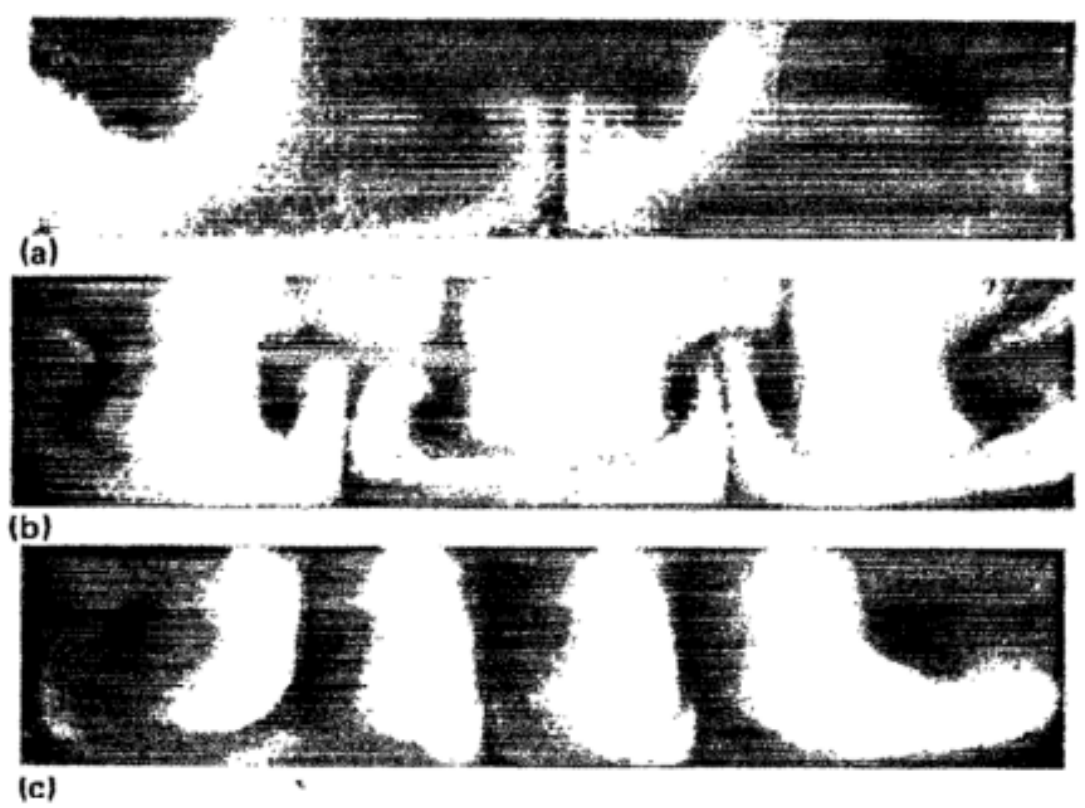

Figura 1.3: (AIDUN; TRIANTAFILLOPOULOS; BENSON, 1991). Estados estacionários. (a) duas células, (b) três células, (c) quatro células.

Através da análise por modos globais não homogêneos em 2 direções, daqui em diante dita biglobal para abreviação, Theofilis, Duck e Owen (2004) identifica e obtém as curvas neutras para os 4 primeiros modos da cavidade para $0 \leq \beta \leq 30$. Dois novos modos são identificados: um modo estacionário $(S 1)$ e outro não estacionário $(T 1)$, ambos com números de onda maiores do que já se havia estudado $(\beta \simeq 15)$. O modo $S 1$ é o dominante, mas aparece apenas como como uma modulação na direção normal ao plano do escoamento base, dificultando sua observação experimental. O modo $T 1$ é o segundo modo mais instável e apresenta boa concordância com o Reynolds crítico observado experimentalmente.

Foram encontrados estudos a respeito do comportamento temporal do escoamento em uma cavidade com tampa deslizante apenas para o caso incompressível. Esta dissertação aborda efeito da compressibilidade na instabilidade hidrodinâmica deste escoamento.

O escoamento base foi resolvido através de simulação numérica direta (DNS) e a metodologia empregada para o estudo da instabilidade hidrodinâmica foi a análise biglobal (THEOFILIS, 2003). Algumas estratégias numéricas foram utilizadas para reduzir o custo computacional necessário à resolução de um problema de autovalor bidimensional. Estas estratégias são o uso de 
matrizes esparsas, redução da ordem do problema por projeções em um subespaço de Krylov e discretização espacial por diferenças finitas. $\mathrm{O}$ uso de diferenças finitas junto com algoritmos baseados em matrizes esparsas garante uma eficiência computacional maior em relação ao problema discretizado por métodos espectrais e utilizando matrizes densas, ainda que precise de mais pontos para atingir a mesma precisão (HEEG; GEURTS, 1997; GENNARO et al., 2013). O algoritmo de Arnoldi (SAAD, 1980) é usado para reduzir a ordem do problema de autovalor, de forma que apenas os autovalores de uma determinada região de interesse do espectro são recuperados.

As curvas neutras do escoamento para diferentes números de Mach foram obtidas através das análises realizadas, revelando a influência da compressibilidade no número de Reynolds crítico. Olhando para o espectro compressível, é possível identificar novos modos que não aparecem em análises incompressíveis. 


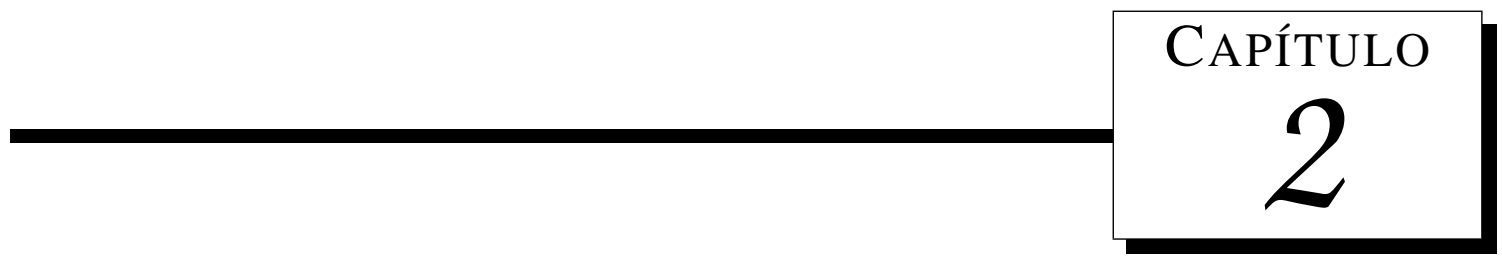

\section{Fundamentos Teóricos}

\subsection{Equações Governantes}

As equações de Navier-Stokes para escoamentos compressíveis foram usadas em sua forma não conservativa $(2.1-2.3)$ para as variáveis primitivas adimensionalizadas: $u, v, w, \rho, e$. A formulação não conservativa foi utilizada pela conveniência ao se aplicar as condições de contorno:

$$
\begin{aligned}
& \frac{\partial \rho}{\partial t}+\rho \frac{\partial u_{i}}{\partial x_{i}}+\frac{\partial \rho}{\partial x_{i}} u_{i}=0, \\
& \frac{\partial u_{j}}{\partial t}-\frac{\partial u_{j}}{\partial x_{i}} u_{i}+\frac{1}{\rho} \frac{\partial p}{\partial x_{j}}-\frac{1}{\rho} \frac{\partial \tau_{i j}}{\partial x_{i}}=0, \\
& \frac{\partial e}{\partial t}+\frac{\partial e}{\partial x_{i}} u_{i}+\frac{p}{\rho} \frac{\partial u_{i}}{\partial x_{i}}-\frac{1}{\rho} \tau_{i j} \frac{\partial u_{j}}{\partial x_{i}}+\frac{1}{\rho} \frac{\partial q_{i}}{\partial x_{i}}=0 .
\end{aligned}
$$

As variáveis adimensionais são definidas por:

$$
\begin{gathered}
\rho=\frac{\rho^{*}}{\rho_{\infty}^{*}}, \quad p=\frac{p^{*}}{p_{\infty}^{*} U_{\infty}^{* 2}}, \quad x_{i}=\frac{x_{i}^{*}}{L^{*}}, \\
T=\frac{T^{*}}{T_{\infty}^{*}}, \quad u_{i}=\frac{u_{i}^{*}}{U_{\infty}^{*}}, \quad t=\frac{t^{*} U_{\infty}^{*}}{L^{*}}, \quad e=\frac{e^{*}}{U_{\infty}^{* 2}},
\end{gathered}
$$

onde $\tau_{i j}$ é o tensor de tensões viscosas e $q_{i}$ é o fluxo de calor, respectivamente definidos por (2.4) e (2.5).

$$
\tau_{i j}=\frac{\mu(T)}{R e}\left[\frac{\partial u_{i}}{\partial x_{j}}+\frac{\partial u_{j}}{\partial x_{i}}-\frac{2}{3} \delta_{i j} \frac{\partial u_{k}}{\partial x_{k}}\right]
$$




$$
q_{i}=-\frac{\mu}{(\gamma-1) \operatorname{RePr} M_{\infty}^{2}} \frac{\partial T}{\partial x_{i}}
$$

A energia interna $e$ é relacionada à temperatura por:

$$
e_{i}=\frac{T}{\left(\gamma^{2}-\gamma\right) M_{\infty}^{2}}
$$

Os números de Reynolds e Prandtl são dados respectivamente por

$$
\begin{gathered}
R e=\frac{\rho_{\infty} U_{\infty} L}{\mu_{\infty}}, \\
\operatorname{Pr}=\frac{\mu_{\infty} c_{p}}{k},
\end{gathered}
$$

onde $k$ é a condutividade térmica.

A hipótese de gás ideal é utilizada como relação constitutiva:

$$
p=(\gamma-1) \rho e
$$

onde $\gamma=c_{p} / c_{v}$ é a razão entre o calor específico a pressão constante e o calor específico a volume constante.

A viscosidade é calculada em função da temperatura pela lei de Sutherland:

$$
\mu(T)=\frac{1+C}{T+C} T^{\frac{3}{2}}, \quad C=\frac{110 K}{T_{\infty}} .
$$

\subsection{Instabilidade Hidrodinâmica Linear}

Escoamentos laminares, quando sujeitos a perturbações podem eventualmente tornar-se turbulentos. Instabilidade hidrodinâmica é o mecanismo de amplificação de pequenas perturbações, que pode levar o escoamento a um regime turbulento ou a um regime laminar diferente do inicial.

Um escoamento é dito estável se qualquer perturbação inicialmente pequena continua limi- 
tada para qualquer tempo decorrido a partir desta condição inicial. O escoamento é instável se alguma perturbação $\mathbf{q}^{\prime}$ inicialmente pequena cresce com o passar do tempo (DRAZIN; REID, 2003). Formalmente o escoamento é estável se, para todo $\varepsilon>0$ existir um $\delta(\varepsilon)$ tal que se

$$
\left\|\mathbf{q}^{\prime}(x, y, z, 0)\right\|<\delta
$$

então

$$
\left\|\mathbf{q}^{\prime}(z, y, z, t)\right\|<\varepsilon
$$

para todo $t>0$.

A teoria de instabilidade hidrodinâmica linear utiliza análise de perturbações para verificar se uma solução das equações de Navier-Stokes é estável ou não. O escoamento é decomposto em uma parcela estacionária, dita escoamento base, e outra não estacionária, com amplitude bem menor que a do escoamento base:

$$
\mathbf{q}(x, y, z, t)=\overline{\mathbf{q}}(x, y, z)+\varepsilon \mathbf{q}^{\prime}(x, y, z, t)
$$

onde $\mathbf{q}=[u, v, w, \rho, e]^{T}$ e $\varepsilon \ll 1$.

Utilizando esta decomposição nas equações de Navier-Stokes e desprezando os termos que contêm produtos de perturbações temos o problema linearizado, cuja solução é expressa pela sobreposição linear dos modos normais, da forma:

$$
\mathbf{q}^{\prime}(x, y, z, t)=\hat{\mathbf{q}}(x, y, z) e^{-i \omega t}
$$

Os autovalores $\omega$ deste problema são complexos e indicam que o escoamento base é instável se a parte imaginária de pelo menos um autovalor for maior que zero, $\omega_{i}>0$. A parte real $\omega_{r}$ é a frequência do modo. Nesta decomposição modal o tempo foi separado das dimensões espaciais. Esta abordagem, conhecida como análise triglobal (THEOFILIS, 2003), não assume nenhuma periodicidade para as perturbações. 


\subsubsection{Teoria Clássica}

A teoria clássica trabalha com escoamentos incompressíveis e paralelos, ou seja, o escoamento base depende apenas de uma única direção. A perturbação é bidimensional ou tridimensional, sendo não homogênea em uma das direções, usualmente $y$ :

$$
\begin{aligned}
& u(x, y, t)=\bar{u}(y)+\varepsilon u^{\prime}(x, y, t), \\
& v(x, y, t)=v^{\prime}(y), \\
& p(x, y, t)=\bar{p}+\varepsilon p^{\prime}(x, y, t),
\end{aligned}
$$

onde

$$
\begin{aligned}
& u^{\prime}(x, y, t)=\hat{u}(y) e^{i(\alpha x-\omega t)}, \\
& v^{\prime}(x, y, t)=\hat{v}(y) e^{i(\alpha x-\omega t)}, \\
& p^{\prime}(x, y, t)=\hat{p}(y) e^{i(\alpha x-\omega t)} .
\end{aligned}
$$

A equação para a perturbação obtida ao substituir (2.15 - 2.17) nas equações de NavierStokes pode ser simplificada na forma que é conhecida como equação de Orr-Sommerfeld:

$$
\alpha(U-c)\left(\frac{d^{2} \hat{v}}{d y^{2}}-\alpha^{2} \hat{v}\right)-\frac{d^{2} U^{\prime \prime}}{d y^{2}} \alpha \hat{v}=-\frac{1}{R e}\left(\frac{d^{4} \hat{v}}{d y^{4}}-2 \alpha^{2} \frac{d^{2} \hat{v}}{d y^{2}}+\alpha^{4} \hat{v}\right)
$$

onde $c$ é a velocidade da onda.

A equação de Orr-Sommerfeld pode ser estendida para considerar perturbações tridimensionais, no entanto, o teorema de Squire (CRIMINALE; JACKSON; JOSLIN, 2003) mostra que o menor Reynolds crítico é obtido para perturbações bidimensionais. Este resultado é válido para escoamentos paralelos, apenas. De fato, no caso da cavidade aqui estudado, observa-se que a instabilidade que ocorre primeiro é a tridimensional. Além disso, o teorema é válido apenas para escoamentos incompressíveis. 


\subsubsection{Análise Temporal e Espacial}

A autofunção $\mathbf{q}^{\prime}$ é complexa e pode-se ter $\alpha$ complexo, $\omega$ complexo, ou ambos complexos (MENDONCA; MEDEIROS, 2009). No caso mais geral tem-se

$$
\mathbf{q}^{\prime}(x, y, t)=\hat{\mathbf{q}}(y) e^{\left(i \alpha_{r} x-i \omega_{r} t\right)} e^{\left(-\alpha_{i} x+\omega_{i} t\right)}
$$

A primeira exponencial é o termo oscilatório e a segunda, o termo da amplitude. Quando $\omega_{i}=0$, a amplitude da perturbação cresce, ou decresce, ao longo de $x$ e a formulação é dita espacial. Neste caso a frequência é $\omega_{i}$, o número de onda em $x$ é $\alpha_{r}$ e a taxa de amplificação espacial é $\alpha_{i}$. Quando $\alpha_{i}=0$, a amplitude da perturbação cresce, ou decresce, com o tempo $t$ e $\omega_{i}$ é a taxa de amplificação temporal. Neste caso a formulação é dita temporal.

Gaster (1962) mostrou que para valores de $\alpha_{i}$ pequenos existe uma relação entre a análise temporal e a espacial, de forma que para uma taxa de amplificação temporal $\omega_{i}$, tem-se uma taxa de amplificação espacial correspondente a

$$
\alpha_{i}=\frac{\omega_{i}}{\frac{d \omega}{d \alpha}}
$$

\subsubsection{Instabilidade Convectiva e Absoluta}

É comum classificar escoamentos paralelos ou quase paralelos em absolutamente instável e convectivamente instável (HUERRE; MONKEWITZ, 1990; DRAZIN; REID, 2003). Se a perturbação cresce ao longo do tempo em um ponto fixo do espaço, a instabilidade é dita absoluta (figura 2.1(b)). Matematicamente

$$
\left\|\mathbf{q}^{\prime}(x, t)\right\| \rightarrow \infty
$$

para $t \rightarrow \infty$. Se, por outro lado, a perturbação cresce ao se propagar na direção do escoamento, mas decresce em um ponto fixo no espaço, ela é dita convectiva (figura 2.1(a)). Neste caso

$$
\left\|\mathbf{q}^{\prime}(x, t)\right\| \rightarrow 0
$$


para $t \rightarrow \infty$, mas existe uma velocidade $V$, tal que

$$
\left\|\mathbf{q}^{\prime}(x+V t, t)\right\| \rightarrow \infty
$$

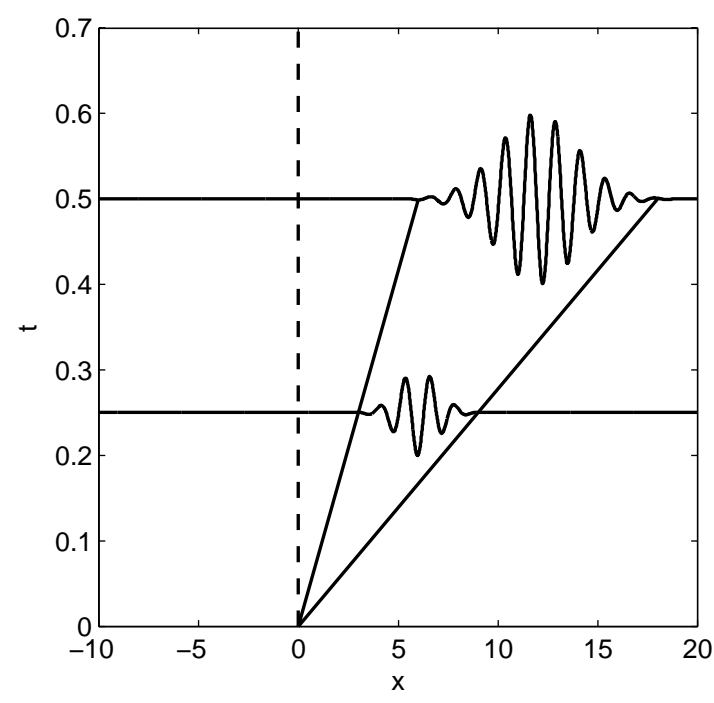

(a)

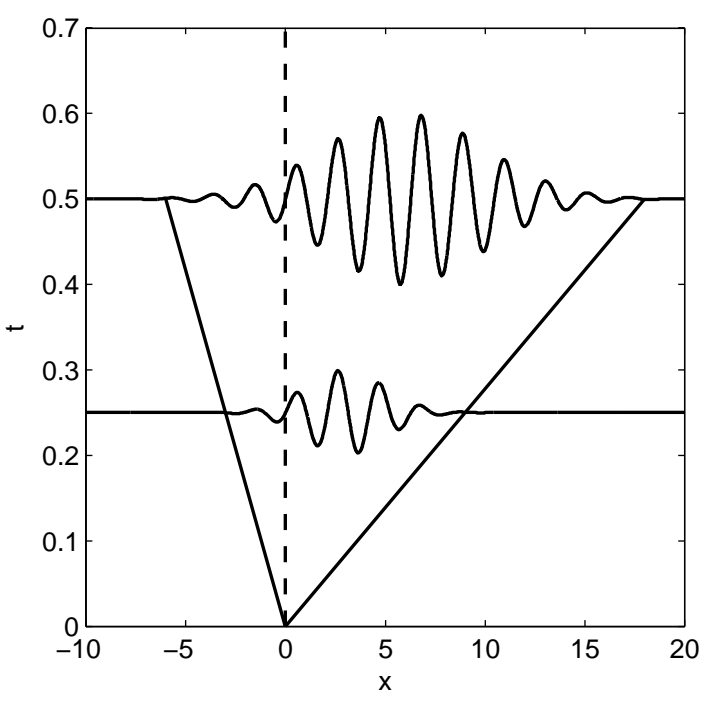

(b)

Figura 2.1: Instabilidade convectiva (a) e absoluta (b).

\subsubsection{Análise Biglobal}

A abordagem utilizada aqui para as análises de instabilidade linear é a biglobal, onde a perturbação tridimensional é assumida como não homogênea em duas direções e periódica em $z$. Esta se desenvolve sobre um escoamento homogêneo na direção $z$ :

$$
\begin{aligned}
\mathbf{q}(x, y, z, t) & =\overline{\mathbf{q}}(x, y)+\varepsilon \mathbf{q}^{\prime}(x, y, z, t), \\
\mathbf{q}^{\prime}(x, y, z, t) & =\hat{\mathbf{q}}(x, y) e^{i(\beta z-\omega t)},
\end{aligned}
$$

onde $\beta \in \mathbb{R}$ é o número de onda na direção $z$.

Substituindo (2.27) e (2.28) nas equações de Navier-Stokes e linearizando o sistema resultante em torno do escoamento base $\hat{\mathbf{q}}$ obtemos as equações para a perturbação, que representam um problema de autovalor generalizado:

$$
\mathscr{L} \hat{\mathbf{q}}=\omega \mathscr{R} \hat{\mathbf{q}}
$$


onde $\mathscr{L}$ e $\mathscr{R}$ são, de forma geral, operadores lineares de segunda ordem:

$$
\mathscr{L}=a_{0}+a_{1} \frac{\partial}{\partial x}+a_{2} \frac{\partial}{\partial y}+a_{3} \frac{\partial^{2}}{\partial x^{2}}+a_{4} \frac{\partial^{2}}{\partial y^{2}}+a_{5} \frac{\partial^{2}}{\partial x \partial y} .
$$

Os elementos dos operadores $\mathscr{L}$ e $\mathscr{R}$ para escoamentos compressíveis indicados abaixo são dados no Apêndice B.

$$
\begin{aligned}
& \mathscr{L}=\left[\begin{array}{ccccc}
\mathscr{L}_{u, u} & \mathscr{L}_{u, v} & \mathscr{L}_{u, w} & \mathscr{L}_{u, p} & \mathscr{L}_{u, T} \\
\mathscr{L}_{v, u} & \mathscr{L}_{v, v} & \mathscr{L}_{v, w} & \mathscr{L}_{v, p} & \mathscr{L}_{v, T} \\
\mathscr{L}_{w, u} & \mathscr{L}_{w, v} & \mathscr{L}_{w, w} & \mathscr{L}_{w, p} & \mathscr{L}_{w, T} \\
\mathscr{L}_{p, u} & \mathscr{L}_{p, v} & \mathscr{L}_{p, w} & \mathscr{L}_{p, p} & \mathscr{L}_{p, T} \\
\mathscr{L}_{T, u} & \mathscr{L}_{T, v} & \mathscr{L}_{T, w} & \mathscr{L}_{T, p} & \mathscr{L}_{T, T}
\end{array}\right], \\
& \mathscr{R}=\left[\begin{array}{ccccc}
\mathscr{R}_{u, u} & 0 & 0 & 0 & 0 \\
0 & \mathscr{R}_{v, v} & 0 & 0 & 0 \\
0 & 0 & \mathscr{R}_{w, w} & 0 & 0 \\
0 & 0 & 0 & \mathscr{R}_{p, p} & \mathscr{R}_{p, T} \\
0 & 0 & 0 & 0 & \mathscr{R}_{T, T}
\end{array}\right]
\end{aligned}
$$

Esta formulação permite uma análise modal da instabilidade hidrodiâmica de escoamentos não paralelos, como é o caso da cavidade, que foi o alvo deste trabalho.

\subsubsection{Não Normalidade do Operador Linear}

O espectro de um operador revela características importantes do fenômeno físico que ele representa. Nos casos abordados aqui, os autovalores indicam se uma perturbação cresce ou decresce quando $t \rightarrow \infty$, sendo que a partir de determinado tempo o fenômeno pode ser representado pelo modo dominante. A análise modal é suficiente para caracterizar o comportamento linear de um operador A quando este é normal, ou seja, quando seus autovetores são ortogonais. É matematicamente equivalente dizer que um operador é normal quando

$$
\mathbf{A} \mathbf{A}^{H}=\mathbf{A}^{H} \mathbf{A}
$$


onde $\mathbf{A}^{H}$ é o conjugado transposto de $\mathbf{A}$.

Entretanto, para operadores não normais, o comportamento físico do fenômeno depende do padrão de cancelamento e sobreposição dos vários modos e não do comportamento de cada modo individualmente. A análise por autovalores depende, implicitamente, de uma transformação para a base formada pelos autovetores. Se a matriz é normal, esta transformação é unitária (rotação ou reflexão). Se a matriz é não normal, esta transformação envolve uma distorção do espaço de estados (TREFETHEN, 1997).

Uma forma de analisar a não normalidade do operador linear é através do pseudo espectro (TREFETHEN et al., 1993). O espectro de uma matriz é dado por

$$
\Lambda(\mathbf{A})=\{\lambda \in \mathbb{C}: \operatorname{det}(\lambda \mathbf{I}-\mathbf{A})=0\}
$$

É equivalente dizer que

$$
\Lambda(\mathbf{A})=\left\{\lambda \in \mathbb{C}:(\lambda \mathbf{I}-\mathbf{A})^{-1} \text { é indefinido }\right\}
$$

Se $\lambda$ é um autovalor de $\mathbf{A}$, por convenção a norma de $(\lambda \mathbf{I}-\mathbf{A})^{-1}$ é dita infinita. O pseudo espectro é o lugar no plano complexo onde $\left\|(\lambda \mathbf{I}-\mathbf{A})^{-1}\right\|$ é grande, porém finita, ou seja,

$$
\Lambda_{\mathcal{\varepsilon}}(\mathbf{A})=\left\{\lambda \in \mathbb{C}:\left\|(\lambda \mathbf{I}-\mathbf{A})^{-1}\right\| \geq \varepsilon^{-1}\right\}
$$

Se a matriz A é normal, o pseudo espectro é representado por círculos de raio $\varepsilon$ em torno dos autovalores. Para matrizes não normais, o pseudo espectro se torna bem mais amplo.

Dois fenômenos importantes observados em operadores não normais são a pseudo ressonância e o crescimento transiente (TREFETHEN et al., 1993). O crescimento transiente é o crescimento de uma perturbação durante um tempo inicial em um fenômeno representado por um operador que apresenta apenas autovalores estáveis. Este fenômeno ocorre graças a sobreposições dos modos não ortogonais (figura 2.2). 

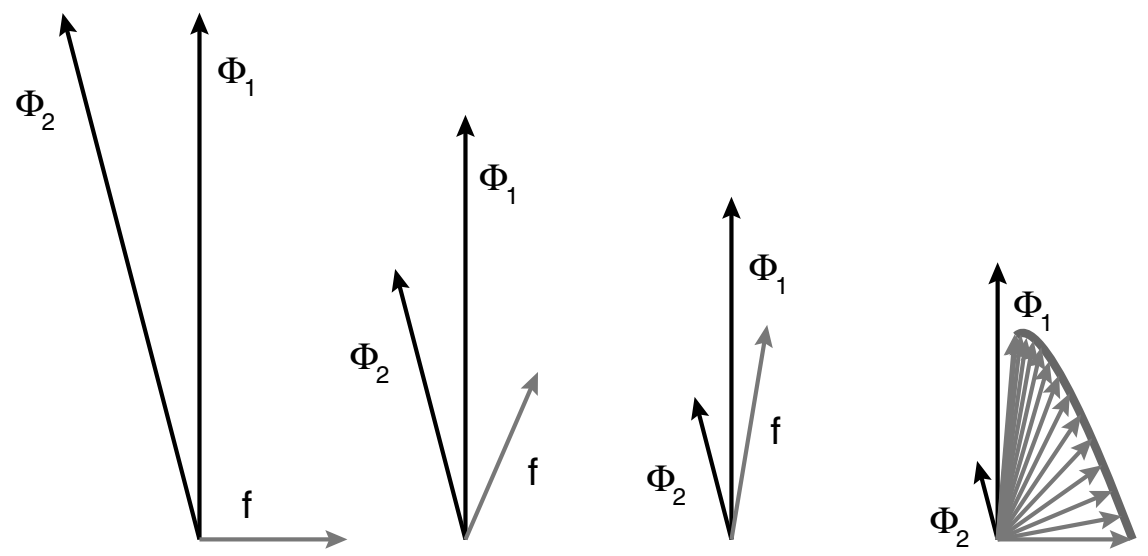

Figura 2.2: (SCHMID, 2007). Crescimento transiente observado entre dois modos $\Phi_{1}$ e $\Phi_{2}$. O vetor $f$ é a diferença entre os dois modos. 


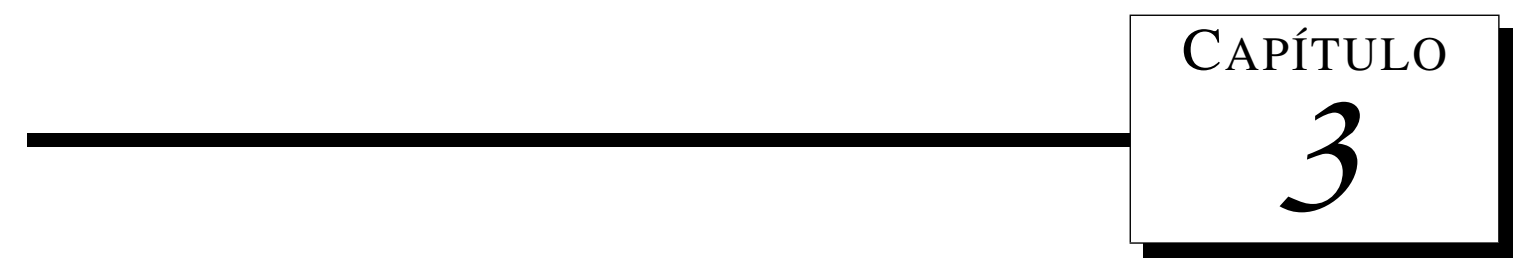

\section{Metodologia}

Para a análise biglobal de instabilidade hidrodinâmica linear, o escoamento é decomposto em uma parcela estacionária bidimensional, dita escoamento base, e perturbações modais tridimensionais, sendo não homogêneas em duas direções e periódicas em uma direção. Estas perturbações podem amplificar ou decair ao longo do tempo e são as soluções de um problema de autovalor generalizado.

O escoamento base foi resolvido através de simulação numérica direta (DNS) e o problema de autovalor para as perturbações foi resolvido através da decomposição QZ de um sistema reduzido pelo método de iteração de Arnoldi.

\subsection{DNS}

Neste trabalho, o código DNS teve a finalidade de gerar um escoamento base para as análises de instabilidade hidrodinâmica linear. No entanto, ele foi desenvolvido de forma a ser usado também para outras atividades do grupo de pesquisa que exigem alta ordem de precisão e a resolução de diferentes escalas, tais como aeroacústica e instabilidade hidrodinâmica não linear. Portanto, foi adotada a discretização espacial por diferenças finitas compactas de alta ordem e com alta resolução espectral (spectral-like). A característica não dissipativa deste tipo de discretização espacial pode gerar instabilidades numéricas em algumas condições. Para contornar este problema, a solução é filtrada em cada passo de tempo por um método de alta ordem. A 
integração temporal é realizada explicitamente pelo método de Runge-Kutta, podendo ser de segunda ou quarta ordem. Malhas estiradas são usadas para aumentar a resolução em certas áreas, ou suprimir vórtices e oscilações antes de uma condição de saída por efeito de dissipação numérica, evitando reflexões. Métricas são utilizadas para que se possa usar as diferenças finitas compactas nestas malhas não uniformes. Estas métricas são calculadas numericamente através do próprio método de diferenças finitas. O código desenvolvido tem capacidade para processamento paralelo, através de uma técnica de decomposição de domínio nas três direções que permite se trabalhar com métodos compactos sem que haja perda de precisão.

\subsubsection{Discretização Espacial}

Lele (1992) propõe um método para diferenças finitas compactas com melhor capacidade de representar os números de onda mais altos, aproximando-se dos métodos espectrais, no entanto permitindo geometrias e condições de contorno mais complexas. A capacidade de representar números de ondas mais altos representa uma redução no erro dispersivo do método.

A forma geral para as diferenças finitas compactas em uma malha uniformemente espaçada é dada pelo sistema linear

$$
\beta f_{i-2}^{\prime}+\alpha f_{i-1}^{\prime}+f_{i}^{\prime}+\alpha f_{i+1}^{\prime}+\beta f_{i+2}^{\prime}=c \frac{f_{i+3}-f_{i-3}}{6 h}+b \frac{f_{i+2}-f_{i-2}}{4 h}+a \frac{f_{i+1}-f_{i-1}}{2 h} .
$$

As relações entre as constantes $a, b, c, \alpha$ e $\beta$ são obtidas pelas séries de Taylor de diferentes ordens:

Segunda ordem: $a+b+c=1+2 \alpha+2 \beta$,

Quarta ordem: $\quad a+2^{2} b+3^{2} c=2 \frac{3 !}{2 !}\left(\alpha+2^{2} \beta\right)$,

Sexta ordem: $\quad a+2^{4} b+3^{4} c=2 \frac{5 !}{4 !}\left(\alpha+2^{4} \beta\right)$,

Oitava ordem: $\quad a+2^{6} b+3^{6} c=2 \frac{7 !}{6 !}\left(\alpha+2^{6} \beta\right)$,

Décima ordem: $\quad a+2^{8} b+3^{8} c=2 \frac{9 !}{8 !}\left(\alpha+2^{8} \beta\right)$.

Utilizando todas as restrições $(3.2$ - 3.6) obtém-se um estêncil com décima ordem de pre- 
cisão. Desta forma todos os coeficientes ficam determinados e a aproximação para a primeira derivada será obtida por um sistema linear pentadiagonal onde o termo não homogêneo para cada ponto é uma combinação linear de sete pontos da função. Também é possível obter ordens de aproximação inferiores usando menos pontos no termo não homogêneo, por exemplo, fazendo $c=0$, ou usando um sistema tridiagonal $(\beta=0)$.

As aproximações para as derivadas modificam o número de onda real obtido por uma diferenciação exata. Através de uma análise de Fourier é possível calcular o número de onda modificado ao resolver as diferenças finitas (3.7). Se, ao invés de obter as diferenças finitas com décima ordem de precisão, utilizarmos as restrições $(3.2-3.3)$ com $a \neq 0, b \neq 0, c \neq 0$, $\alpha \neq 0$ e $\beta \neq 0$, teremos ainda três graus de liberdade para o estêncil. Usando a relação (3.7) é possível fixar três números de onda que permanecerão exatos na derivação aproximada (3.8 3.10). Este é o método usado por Lele (1992) e resulta em um sistema linear pentadiagonal.

$$
\begin{gathered}
w^{\prime}(w)=\frac{a \sin (w)+(b / 2) \sin (2 w)+(c / 3) \sin (3 w)}{1+2 \alpha \cos (w)+2 \beta \cos (2 w)} . \\
w^{\prime}\left(w_{1}\right)=w_{1}, \\
w^{\prime}\left(w_{2}\right)=w_{2}, \\
w^{\prime}\left(w_{3}\right)=w_{3} .
\end{gathered}
$$

Um método muito similar foi utilizado para a discretização espacial neste código DNS. A diferença consiste em fazer $\beta=0$ e fixar dois números de onda para serem exatos $(3.8-3.9)$. Isto reduz um pouco a resolução espectral, mas resulta em um sistema linear tridiagonal, o que é vantajoso computacionalmente. Também foi implementado um estêncil que utiliza cinco pontos da função para o termo não homogêneo do sistema ao invés de sete. Nesse caso apenas um número de onda é fixado para ser exato.

O número de onda modificado $w^{\prime}$ em função no número de onda real $w$ é mostrado na figura 3.1 para estêncil com cinco e sete pontos, ambos para um sistema tridiagonal. Ambos 
os métodos possuem quarta ordem de precisão. Na mesma figura é mostrado o número de onda modificado para diferenças finitas compactas de sexta ordem de precisão, porém sem utilizar o método para aumentar a resolução espectral. As condições impostas para aumentar a resolução espectral são mostradas nas tabelas 3.1 e 3.2 para estêncil com cinco e sete pontos, respectivamente.

Tabela 3.1: Restrições para estêncil com cinco pontos

\begin{tabular}{cc}
\hline \hline Caso & Restrição \\
\hline 1 & $w^{\prime}(1,5)=1,5$ \\
2 & $w^{\prime}(1,8)=1,8$ \\
3 & $w^{\prime}(2,2)=2,2$ \\
4 & $w^{\prime}(2,4)=2,4$ \\
\hline
\end{tabular}

Tabela 3.2: Restrições para estêncil com sete pontos

\begin{tabular}{ccc}
\hline \hline Caso & Restrição 1 & Restrição 2 \\
\hline 1 & $w^{\prime}(2,3)=2,3$ & $w^{\prime}(2,4)=2,4$ \\
2 & $w^{\prime}(2,4)=2,4$ & $w^{\prime}(2,5)=2,5$ \\
3 & $w^{\prime}(2,5)=2,5$ & $w^{\prime}(2,6)=2,6$
\end{tabular}

Outra forma de avaliar o erro dispersivo das diferenças finitas é através da velocidade de fase (3.12) das ondas propagadas pela equação de convecção (3.11). As velocidades de fase em função do número de onda são mostradas na figura 3.2 e comparadas com diferenças finitas compactas tradicionais de sexta ordem. As diferenças finitas utilizadas para as simulações deste trabalho foram obtidas com as restrições do primeiro caso da tabela 3.2.

$$
\begin{gathered}
\frac{\partial f}{\partial t}+c \frac{\partial f}{\partial x}=0 . \\
c_{p h}=\frac{w^{\prime}(w)}{w} .
\end{gathered}
$$

A ordem de precisão dos métodos foi verificada numericamente através da comparação da derivada numérica da função $f=\sin (x)$ com seu resultado analítico. Como esperado, os 


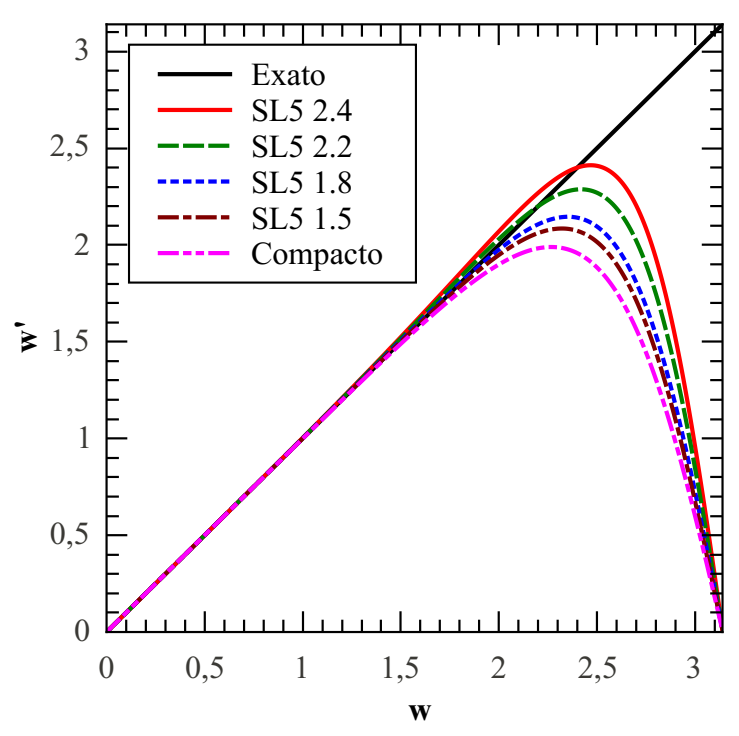

(a) Estêncil de cinco pontos.

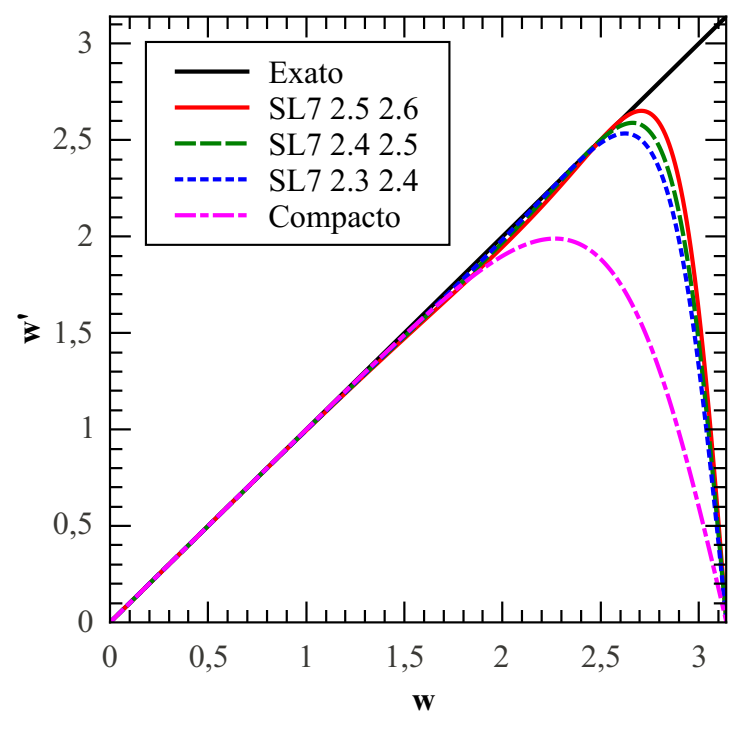

(b) Estêncil de sete pontos.

Figura 3.1: Número de onda modificado para diferenças finitas compactas com alta resolução espectral utilizando as restrições das tabelas 3.1 e 3.2.

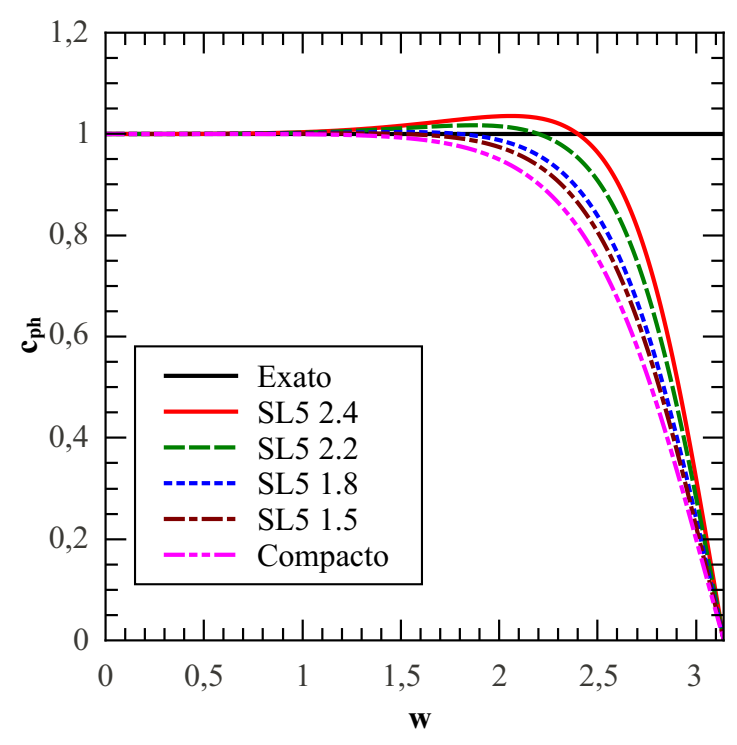

(a) Estêncil de cinco pontos.

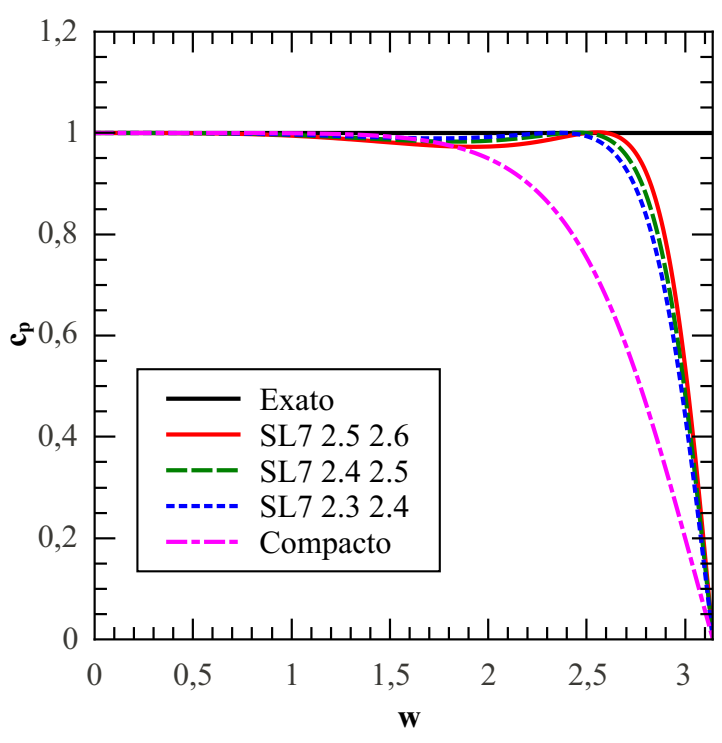

(b) Estêncil de sete pontos.

Figura 3.2: Velocidade de fase para diferenças finitas compactas com alta resolução espectral utilizando as restrições das tabelas 3.1 e 3.2. 


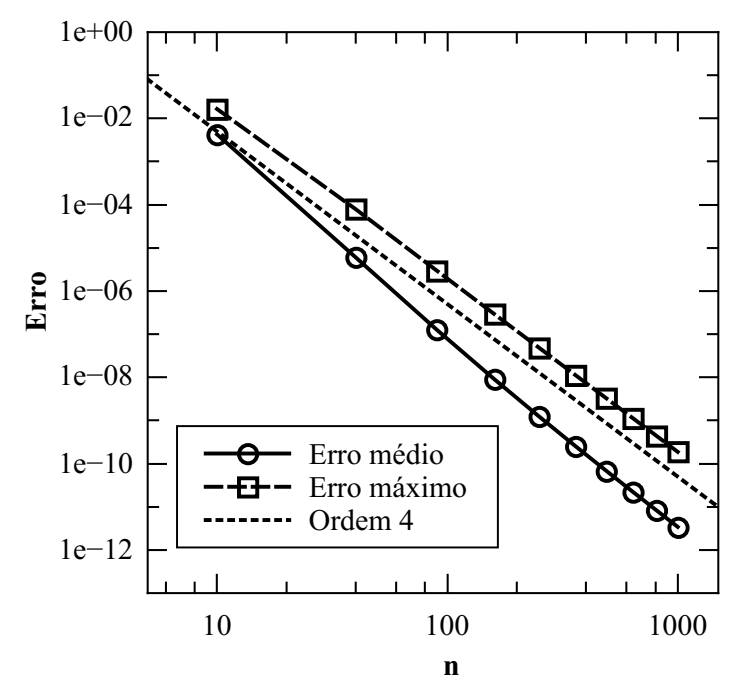

(a) Estêncil de cinco pontos.

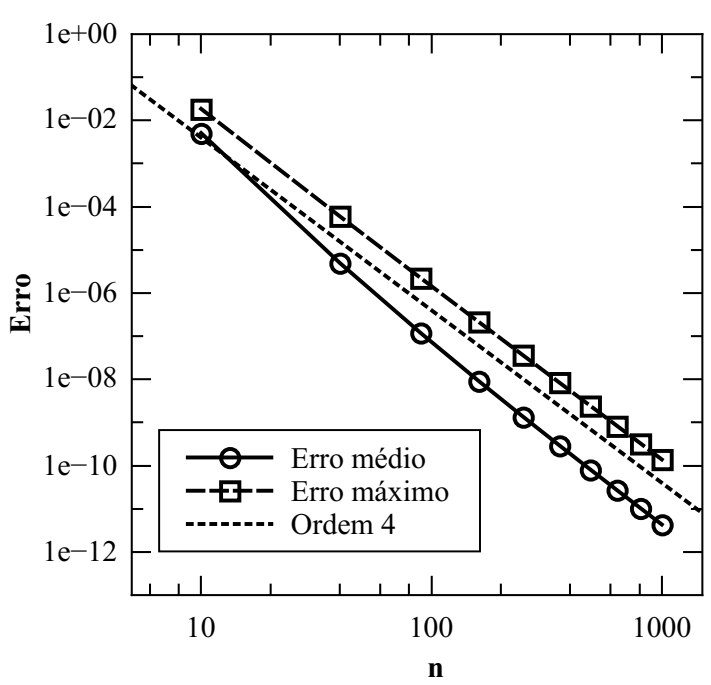

(b) Estêncil de sete pontos.

Figura 3.3: Ordem de precisão para diferenças finitas compactas com alta resolução espectral utilizando as restrições das tabelas 3.1 e 3.2.

métodos apresentam quarta ordem de precisão.

A estabilidade das diferenças finitas foi avaliada através da análise de estabilidade linear da equação de convecção (3.11). A equação (3.11) foi discretizada para o intervalo $x=[0,1]$ com condições de contorno $f(x=0, t)=0$, originando o sistema

$$
\mathbf{A} \hat{\mathbf{f}}^{\prime}=\frac{1}{\Delta x} \hat{\mathbf{f}}
$$

Admitindo solução por modos normais $\hat{\mathbf{f}}=\exp (\omega t) \tilde{\mathbf{f}}$, o sistema (3.13) se transforma em um problema de autovalor:

$$
-\frac{\omega \Delta x}{c} \tilde{\mathbf{A f}}=\mathbf{B} \tilde{\mathbf{f}}
$$

A estabilidade é verificada uma vez que todos os autovalores $\omega^{\prime}=\omega \Delta x / c$ possuem parte real negativa (figura 3.4).

A figura 3.5 mostra a vantagem computacional de se usar as diferenças finitas aqui propostas ao invés de resolver o sistema pentadiagonal apresentado por Lele (1992). O erro foi obtido pela comparação entre a derivada numérica da função $f=\operatorname{sen}(x)$ e sua derivada analítica em um ponto no interior do domínio. Ambos os métodos tem quarta ordem de precisão. Embora 


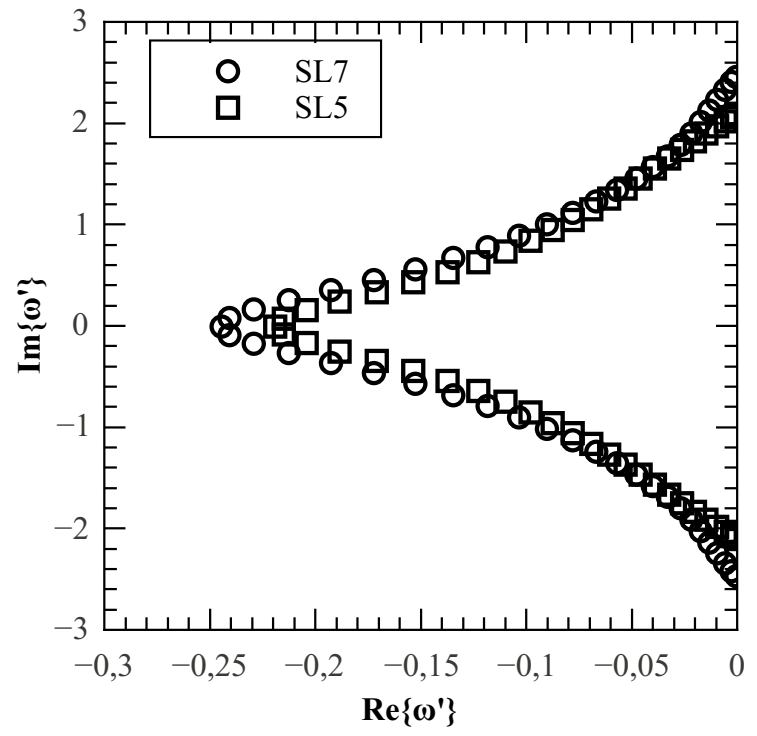

(a) Autovalores.

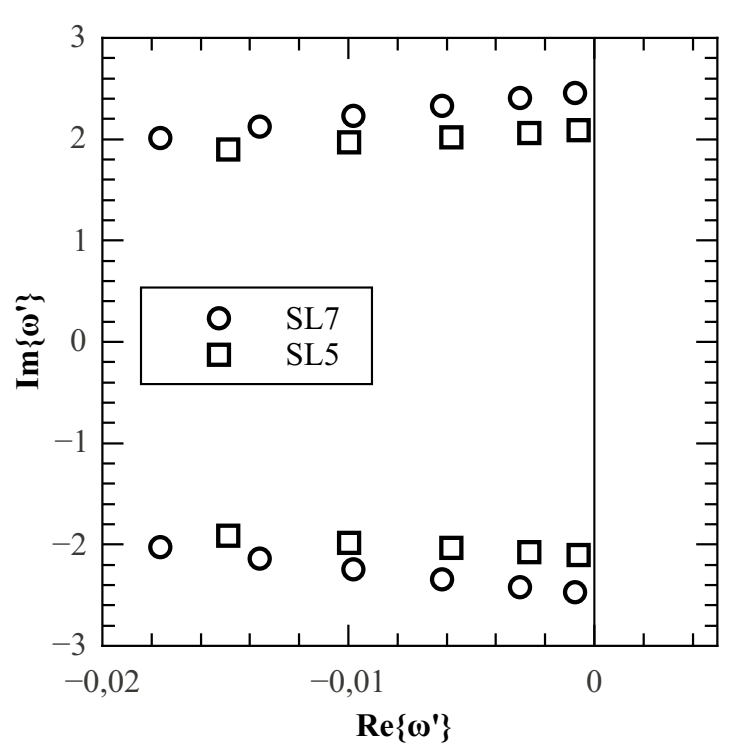

(b) Destaque na região próxima de $\operatorname{Re}\left\{\omega^{\prime}\right\}=0$.

Figura 3.4: Autovalores da equação de convecção discretizada.

o sistema pentadiagonal garanta uma precisão melhor que o sistema tridiagonal para o mesmo número de pontos, o sistema tridiagonal é capaz de obter a mesma precisão com um tempo computacional menor, ainda que utilize mais pontos no domínio para isso.

Para os pontos das fronteiras com condições de contorno não periódicas é utilizado um estêncil descentrado com quarta ordem de precisão:

$$
f_{1}^{\prime}+\alpha f_{2}^{\prime}=\frac{1}{h}\left(a f_{1}+b f_{2}+c f_{3}+d f_{4}\right)
$$

Os coeficientes são apresentados na tabela 3.3.

Tabela 3.3: Coeficientes para diferenças finitas compactas descentradas de quarta ordem.

\begin{tabular}{ccccc}
\hline \hline$\alpha$ & $a$ & $b$ & $c$ & $d$ \\
\hline 3 & $-17 / 6$ & $3 / 1$ & $3 / 2$ & $-1 / 6$
\end{tabular}

A segunda derivada foi calculada aplicando-se duas vezes o método de diferenças finitas para a primeira derivada. Da mesma forma, as derivadas cruzadas foram calculadas com aplicações sucessivas do método, uma para cada direção. 


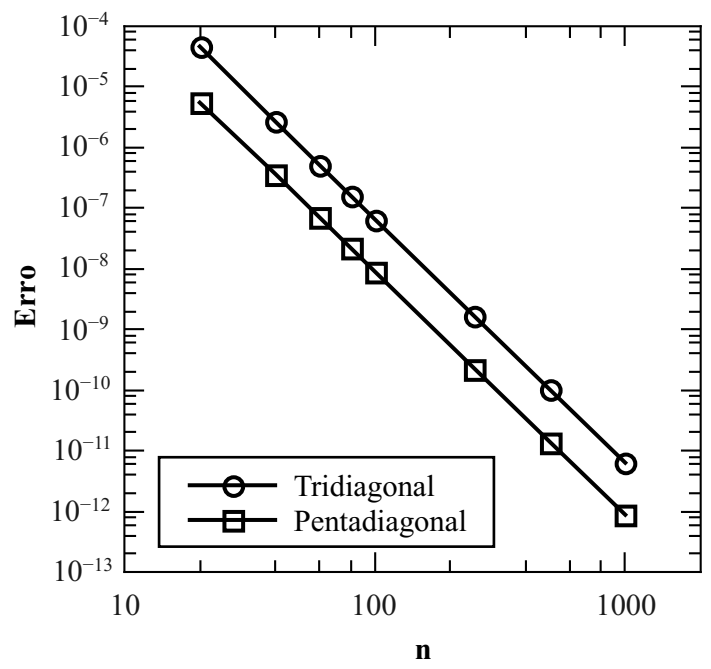

(a) Erro para um ponto no interior do domínio.

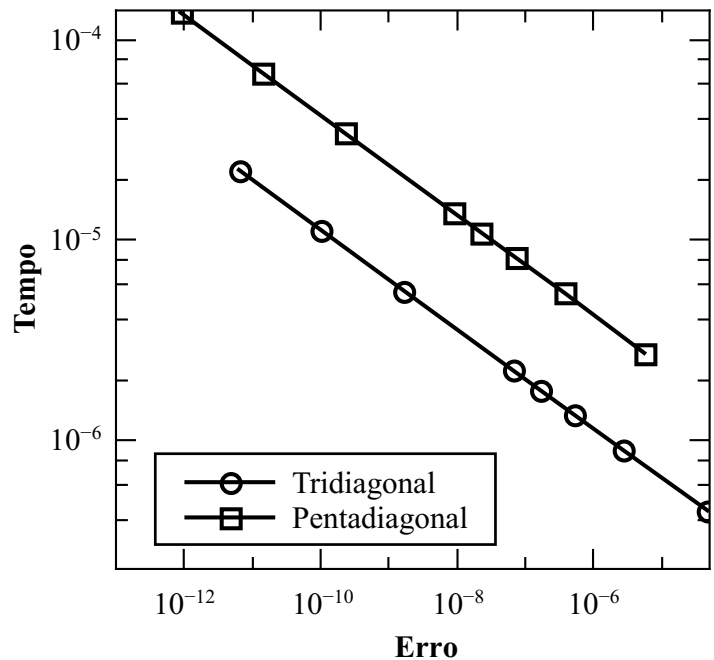

(b) Tempo computacional para se obter a precisão desejada.

Figura 3.5: Comparação do método proposto com as diferenças finitas obtidas por Lele (1992).

\subsubsection{Condições de Contorno}

Quatro condições de contorno básicas foram implementadas possibilitando a solução dos diferentes escoamentos que pretendemos simular com o código DNS. São elas: Dirichlet, Neumann, extrapolação e periodicidade, além de uma condição de compatibilidade para a pressão.

A aplicação da condição de contorno de Dirichlet é trivial, bastando impor $f_{1}=c$.

A condição de contorno de Neumann é obtida através de diferenças finitas não compactas, do tipo

$$
f_{i}^{\prime}=\sum_{i=1}^{n} a_{i} f_{i}=c
$$

onde $a_{i}$ são os coeficientes das diferenças finitas descentradas de ordem $n-1$.

O valor da função no ponto $i=1$ para satisfazer $f_{1}^{\prime}=c$ é dado por

$$
f_{1}=\frac{c-\sum_{i=2}^{n} a_{i} f_{i}}{a_{1}} .
$$

Foram usadas diferenças finitas explícitas de quarta ordem. Os respectivos coeficientes são dados na tabela 3.4 . 
Tabela 3.4: Coeficientes para diferenças finitas não compactas descentradas de quarta ordem.

\begin{tabular}{ccccc}
\hline \hline$a_{1}$ & $a_{2}$ & $a_{3}$ & $a_{4}$ & $a_{5}$ \\
\hline-25 & 48 & -36 & 16 & -3
\end{tabular}

A condição de contorno de extrapolação é obtida pelo polinômio de Lagrange de ordem $n-1:$

$$
f_{1}=\sum_{i=1}^{n} \prod_{i=1}^{n} \frac{x_{1}-x_{j}}{x_{i}-x_{j}} f_{i}, \text { para } i \neq j
$$

Verificou-se que esta condição normalmente precisa ter alta ordem para que haja estabilidade. Bons resultados foram obtidos para oitava ordem.

Algumas vezes é necessário usar a condição de periodicidade. Quando aplicada, esta condição faz com que o sistema deixe de ser tridiagonal:

$$
\overline{\mathbf{A}} \mathbf{f}^{\prime}=\mathbf{B f},
$$

onde

$$
\overline{\mathbf{A}}=\left[\begin{array}{cccccccc}
b_{1} & c_{1} & 0 & 0 & 0 & \cdots & 0 & a_{1} \\
a_{2} & b_{2} & c_{2} & 0 & 0 & \cdots & 0 & 0 \\
0 & a_{3} & b_{3} & c_{3} & 0 & \cdots & 0 & 0 \\
\vdots & \vdots & \vdots & \vdots & \vdots & \ddots & \vdots & \vdots \\
0 & 0 & 0 & 0 & \cdots & a_{n-1} & b_{n-1} & c_{n-1} \\
c_{n} & 0 & 0 & 0 & \cdots & 0 & a_{n} & b_{n}
\end{array}\right] .
$$

Para que o mesmo algoritmo usado para matrizes tridiagonais possa ser aplicado, o sistema (3.19) é convertido em dois sistemas tridiagonais através da formula de Shermann-Morrison:

$$
\overbrace{\left(\mathbf{A}+\mathbf{u v}^{T}\right)}^{\overline{\mathbf{A}}} \mathbf{f}^{\prime}=\mathbf{B f},
$$


onde A é uma matriz tridiagonal:

$$
\begin{gathered}
\mathbf{A}=\left[\begin{array}{cccccccc}
0 & c_{1} & 0 & 0 & 0 & \cdots & 0 & 0 \\
a_{2} & b_{2} & c_{2} & 0 & 0 & \cdots & 0 & 0 \\
0 & a_{3} & b_{3} & c_{3} & 0 & \cdots & 0 & 0 \\
\vdots & \vdots & \vdots & \vdots & \vdots & \ddots & \vdots & \vdots \\
0 & 0 & 0 & 0 & \cdots & a_{n-1} & b_{n-1} & c_{n-1} \\
0 & 0 & 0 & 0 & \cdots & 0 & a_{n} & b_{n}-\frac{c_{n} a_{1}}{b_{1}}
\end{array}\right], \\
\mathbf{u}=\left[\begin{array}{lllllc}
b 1 & 0 & 0 & \cdots & 0 & c_{n}
\end{array}\right]^{T}, \\
\mathbf{v}=\left[\begin{array}{llllll}
1 & 0 & 0 & \cdots & 0 & a_{1} / b_{1}
\end{array}\right]^{T} .
\end{gathered}
$$

A solução de (3.19) é obtida resolvendo

$$
\mathbf{A y}=\mathbf{B f}
$$

$$
\mathbf{A q}=\mathbf{u}
$$

$$
\mathbf{f}^{\prime}=\mathbf{y}-\frac{\mathbf{v}^{T} \mathbf{y}}{\left(1+\mathbf{v}^{T} \mathbf{q}\right)} \mathbf{q} .
$$

Para a pressão nas paredes, é necessário utilizar uma condição de contorno artificial. Escoamentos incompressíveis com alto número de Reynolds permitem, como simplificação, o uso de condições de contorno de Neumann. Nos casos onde isto não é aplicável uma condição de contorno de compatibilidade pode ser obtida para a pressão a partir da equação de momento (Navier-Stokes) em regime estacionário:

$$
\frac{\partial p}{\partial x_{j}}-\frac{p}{(\gamma-1) e} \frac{\partial u_{j}}{\partial x_{i}} u_{i}=\frac{\partial \tau_{i j}}{\partial x_{i}}
$$

Os valores para $u_{j}, \frac{\partial u_{j}}{\partial x_{i}}, u_{i}$ e $\frac{\partial \tau_{i j}}{\partial x_{i}}$ são obtidos do instante anterior com as condições de 
contorno físicas já aplicadas. A condição para a compatibilidade (3.28) é uma condição de contorno de Robin, ou seja, envolve uma combinação linear entre a função e sua derivada:

$$
\alpha f_{1}^{\prime}+\beta f_{1}=c
$$

A aplicação desta condição dá-se de forma similar à condição de Neumann, através de diferenças finitas não compactas de quarta ordem $(n=5)$ :

$$
f_{1}=\frac{c-\alpha \sum_{i=2}^{n} a_{i} f_{i}}{\alpha a_{1}+\beta}
$$

onde os coeficientes $a_{i}$ são dados pela tabela 3.4,

$$
\begin{aligned}
\alpha & =1 \\
\beta & =-\frac{1}{(\gamma-1) e} \frac{\partial u_{j}}{\partial x_{i}} u_{i} \\
c & =\frac{\partial \tau_{i j}}{\partial x_{i}}
\end{aligned}
$$

\subsubsection{Integração Temporal}

Para a integração temporal foi utilizado o método de Runge-Kutta de quarta ordem:

$$
y_{n+1}=y_{n}+\frac{1}{6}\left(K_{1}+K_{2}+K_{3}+K_{4}\right)
$$

onde

$$
\begin{aligned}
& K_{1}=\Delta t f\left(t_{n}, y_{n}\right), \\
& K_{2}=\Delta t f\left(t_{n}+\frac{1}{2} \Delta t, y_{n}+\frac{1}{2} K_{1}\right), \\
& K_{3}=\Delta t f\left(t_{n}+\frac{1}{2} \Delta t, y_{n}+\frac{1}{2} K_{2}\right), \\
& K_{4}=\Delta t f\left(t_{n}+\Delta t, y_{n}+K_{3}\right) .
\end{aligned}
$$

Este método é muito popular em aplicações semelhantes e possui a precisão adequada. Por ser um método explícito, apresenta estabilidade condicional. Dois critérios de estabilidade são 
verificados antes da integração:

- Critério de estabilidade para convecção

$$
\Delta t \leq \frac{C F L}{\left(\frac{\frac{1}{M_{\infty}}+u_{\max }}{\Delta x}+\frac{\frac{1}{M_{\infty}}+v_{\max }}{\Delta y}+\frac{\frac{1}{M_{\infty}}+w_{\max }}{\Delta z}\right)}
$$

onde $C F L$ é o número de Courant-Friedrichs-Lewy e depende dos métodos de integração temporal e discretização espacial.

- Critério de estabilidade para difusão

$$
\Delta t \leq \frac{1}{\frac{6}{R e}\left(\frac{1}{\Delta x^{2}}+\frac{1}{\Delta y^{2}}+\frac{1}{\Delta z^{2}}\right)} .
$$

Para simulações onde a solução transiente não é de interesse, o método de Runge-Kutta de quarta ordem não é necessário. Para estes casos também foi implementado o método de RungeKutta de segunda ordem, que demanda a metade do custo computacional do método de quarta ordem:

$$
y_{n+1}=y_{n}+K_{2}
$$

\subsubsection{Métrica}

Os métodos de diferenças finitas utilizados neste trabalho são obtidos para malhas uniformes. No entanto, frequentemente é necessário trabalhar com malhas mais refinadas em determinadas regiões ou com malhas menos refinadas para promover a relaminarização do escoamento por efeito de dissipação numérica.

O conceito de métrica permite trabalhar com malhas curvilíneas e variantes no tempo. No entanto, neste trabalho foram consideradas apenas malhas cartesianas retilíneas e invariantes no tempo, porém não uniformes. O domínio físico não uniforme $(x, y, z)$ é mapeado para um domínio computacional uniforme $(\xi, \eta, \zeta)$, onde $0 \leq \xi, \eta, \zeta \leq 1$. Os métodos de diferenças finitas descritos anteriormente são válidos neste domínio computacional. Como a malha do domínio físico é cartesiana e retilínea, a transformação para o domínio computacional é dada 
por funções que dependem apenas de uma direção:

$$
\begin{gathered}
\xi=\xi(x), \\
\eta=\eta(y), \\
\zeta=\zeta(z) .
\end{gathered}
$$

As derivadas no domínio físico são expressas por:

$$
\begin{aligned}
& \frac{\partial f}{\partial \xi}=\frac{\partial f}{\partial x} \frac{\partial x}{\partial \xi} \\
& \frac{\partial f}{\partial \eta}=\frac{\partial f}{\partial y} \frac{\partial y}{\partial \eta} \\
& \frac{\partial f}{\partial \zeta}=\frac{\partial f}{\partial z} \frac{\partial z}{\partial \zeta}
\end{aligned}
$$

onde $\frac{\partial x}{\partial \xi}, \frac{\partial y}{\partial \eta}$ e $\frac{\partial x}{\partial \zeta}$ são as métricas.

As métricas são calculadas numericamente através de diferenças finitas e são aplicadas no lado esquerdo do sistema das diferenças finitas compactas (3.1). Para a direção $x$, obtém-se:

$$
\begin{aligned}
& {\left[\beta\left(\frac{\partial x}{\partial \xi}\right)_{i-2}\right]\left(\frac{\partial f}{\partial x}\right)_{i-2}+\left[\alpha\left(\frac{\partial x}{\partial \xi}\right)_{i-1}\right]\left(\frac{\partial f}{\partial x}\right)_{i-1}+\left(\frac{\partial x}{\partial \xi}\right)_{i}\left(\frac{\partial f}{\partial x}\right)_{i}+} \\
& {\left[\alpha\left(\frac{\partial x}{\partial \xi}\right)_{i+1}\right]\left(\frac{\partial f}{\partial x}\right)_{i+1}+\left[\beta\left(\frac{\partial x}{\partial \xi}\right)_{i+2}\right]\left(\frac{\partial f}{\partial x}\right)_{i+2}=} \\
& c \frac{f_{i+3}-f_{i-3}}{6 h}+b \frac{f_{i+2}-f_{i-2}}{4 h}+a \frac{f_{i+1}-f_{i-1}}{2 h}
\end{aligned}
$$

onde $h$ é o espaçamento constante da malha computacional.

De forma análoga, são obtidas as diferenças finitas para malhas não uniformes nas demais direções.

\subsubsection{Filtro}

Não linearidades, estiramento de malha, condições de contorno, entre outros fatores, levam ao surgimento de ondas espúrias com o máximo número de onda que a malha é capaz de resolver. Como as diferenças finitas centradas não apresentam erro dissipativo, estas ondas podem ser amplificadas e contaminar a solução numérica. Para eliminar este problema, foi utilizado 
um filtro compacto de alta ordem proposto por Gaitonde e Visbal (1998). Este filtro atua apenas nos altos números de onda, que são mal resolvidos pela malha e não comprometem a solução física do escoamento. Além disso, para malhas suficientemente refinadas é possível desativar o filtro, pois a viscosidade já é suficiente para impedir o desenvolvimento das ondas espúrias.

Para os pontos interiores, a solução filtrada $\bar{\phi}$ é dada pelo sistema linear:

$$
\alpha_{f} \bar{\phi}_{i-1}+\bar{\phi}_{i}+\alpha_{f} \bar{\phi}_{i+1}=\sum_{n=0}^{N} \frac{a_{n}}{2}\left(\phi_{i+n}+\phi_{i-n}\right) .
$$

O parâmetro $-0,5 \leq \alpha_{f} \leq 0,5$ controla a atuação do filtro. Aumentando $\alpha_{f}$ o filtro atua em uma parte menor do espectro, ou seja, sua intensidade é reduzida. Para a geração dos escoamentos base da cavidade foi usado $\alpha=0,4$. Além do parâmetro $\alpha_{f}$, a ordem do filtro também controla sua intensidade. Quanto maior a ordem, menor será a filtragem. Os coeficientes $a_{n}$ para um filtro de décima ordem são apresentados na tabela 3.5. O filtro de décima ordem foi implementado para garantir a mínima filtragem possível, impedindo a distorção da solução física.

Tabela 3.5: Coeficientes para o filtro de décima ordem.

\begin{tabular}{cccccc}
\hline \hline$a_{0}$ & $a_{1}$ & $a_{2}$ & $a_{3}$ & $a_{4}$ & $a_{5}$ \\
\hline$\frac{193+126 \alpha_{f}}{256}$ & $\frac{105+302 \alpha_{f}}{256}$ & $15 \frac{-1+2 \alpha_{f}}{64}$ & $45 \frac{1-2 \alpha_{f}}{512}$ & $5 \frac{-1+2 \alpha_{f}}{256}$ & $\frac{1-2 \alpha_{f}}{512}$ \\
\hline
\end{tabular}

Para os pontos próximos às fronteiras, o filtro pode ter sua ordem reduzida, ou estêncis descentrados podem ser usados. Ao reduzir a ordem do filtro, é necessário utilizar um refinamento maior para garantir a mesma performance. Os estêncis descentrados podem amplificar alguns números de onda, levando à instabilidade. Optou-se por não filtrar os pontos próximos às fronteiras e apenas o estêncil centrado foi usado.

A resposta em frequência do filtro em função do número de onda $T(w)$ foi calculada numericamente aplicando a filtragem à função impulso e calculando o módulo da transformação de Fourier da função filtrada. O resultado é apresentado na figura 3.6. 


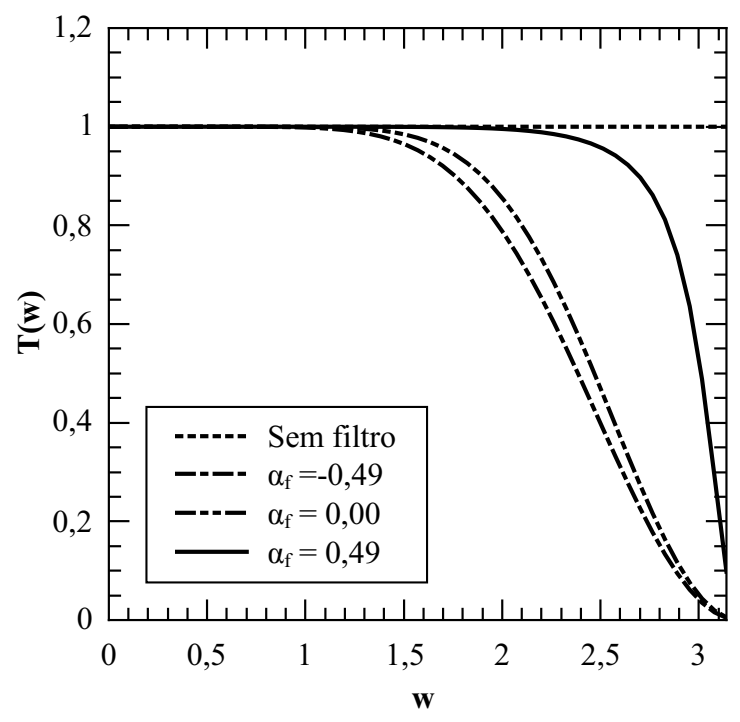

Figura 3.6: Função de resposta em frequência do filtro compacto.

\subsubsection{Zona de Amortecimento}

Escoamentos onde grandes perturbações são desenvolvidas e propagadas até a saída apresentam um problema de instabilidade numérica. As ondas de vorticidade e as ondas acústicas são refletidas na saída do escoamento, contaminando a solução. É preciso utilizar algum método para remover estas ondas antes que atinjam a saída. A supressão destas ondas foi efetuada apenas pelo estiramento da malha, promovendo um efeito de dissipação numérica. A malha estirada deixa de capturar as escalas destas perturbações e estas são naturalmente removidas do escoamento.

O estiramento da malha deve ser feito de forma suave, caso contrário ondas espúrias surgem no início da zona de amortecimento. O estiramento (3.50) foi aplicado através de um acréscimo $\delta_{x}$ sobre a malha $x$ a partir de um ponto onde a malha original possui espaçamento $\delta_{1}$. A zona de amortecimento possui tamanho $L_{b}$ e é parametrizada pela coordenada $0 \leq x_{b} \leq L_{b}$. O parâmetro $K_{b}$ controla o tamanho e o estiramento da zona de amortecimento. O parâmetro $\lambda_{b}$ controla a suavidade do estiramento, sendo $\lambda_{b}=1$ um valor típico.

$$
\delta_{x}=\left\{\frac{K_{b}}{1+\exp \left[\lambda_{b}\left(10-12 \frac{x_{b}}{L_{b}}\right)\right]}+1\right\} \delta_{1} .
$$

Para verificar se a zona de amortecimento era capaz de suprimir as ondas de vorticidade e 


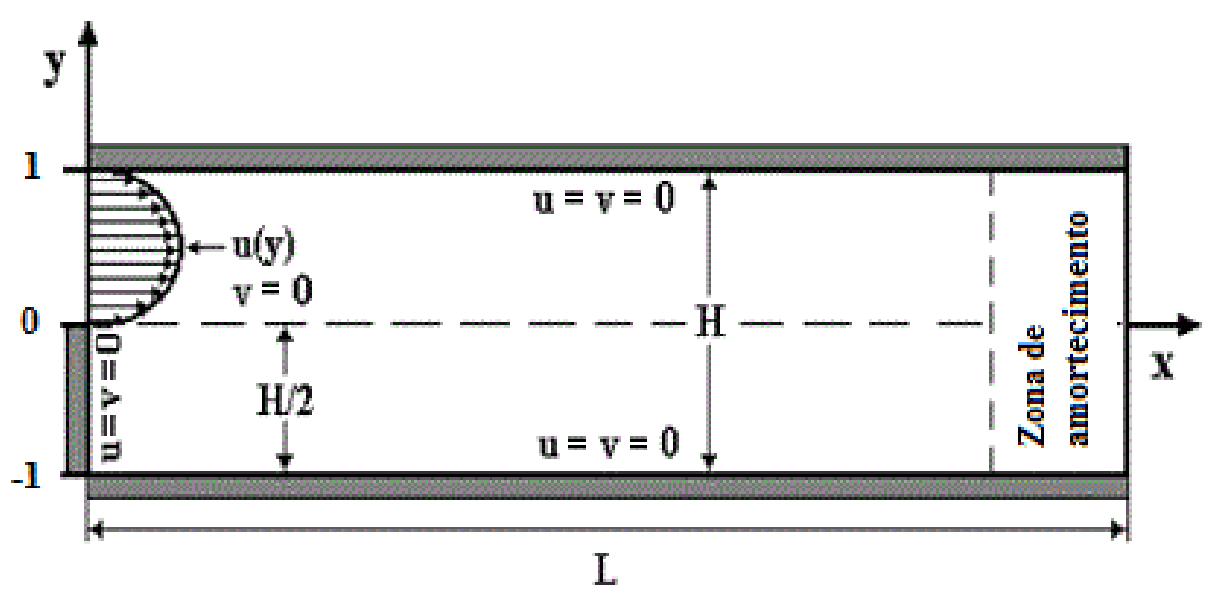

Figura 3.7: Geometria da expansão abrupta.

evitar a instabilidade numérica, foi simulado um escoamento confinado sofrendo uma expansão abrupta a $R e=600$ e $M_{\infty}=0,2$ com e sem buffer zone (figura 3.7).

A condição de contorno na entrada foi um escoamento parabólico em metade do domínio para $u$, conforme a figura $3.8, v=0, T=1$ e extrapolação para $p$. Na saída todas as variáveis foram extrapoladas, exceto a pressão, que foi fixada em $p=\frac{1}{\gamma M_{\infty}^{2}}$. Nas paredes, inclusive em $(x=0 ;-1 \leq y \leq 0)$, foi imposta a condição de não escorregamento, ou seja, $u=v=0$, condição isotérmica, $e=\frac{1}{\left(\gamma^{2}-\gamma\right) M_{\infty}^{2}}$, e condição de compatibilidade para a pressão.

O estiramento usado foi suficiente para suprimir as ondas de vorticidade e evitar as instabilidades numéricas, como pode ser visto na figura 3.9, pelos contornos da velocidade $v$ para o escoamento sem e com zona de amortecimento. A zona de amortecimento não é mostrada na figura.

No caso sem zona de amortecimento, o escoamento não atingiu um estado estacionário, permanecendo periódico. 


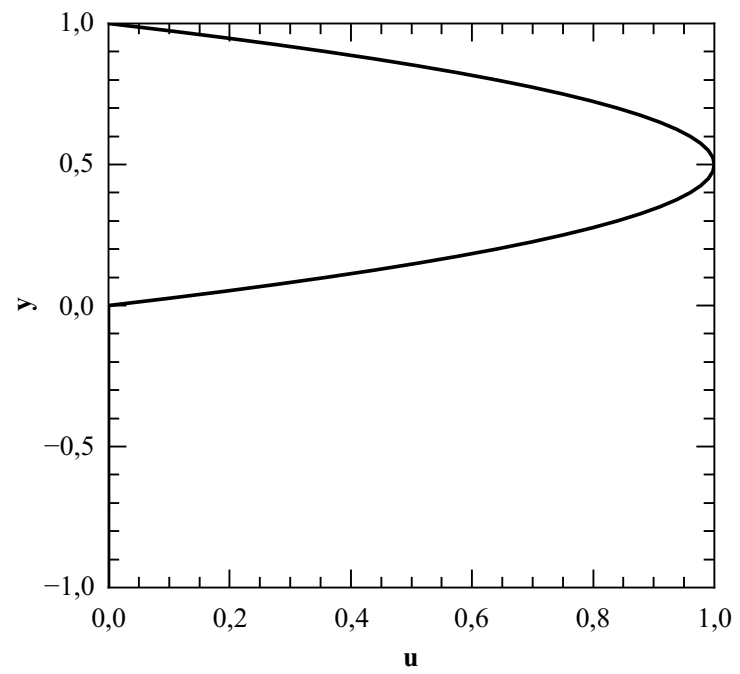

Figura 3.8: Perfil de velocidade $u$ na entrada da expansão abrupta.

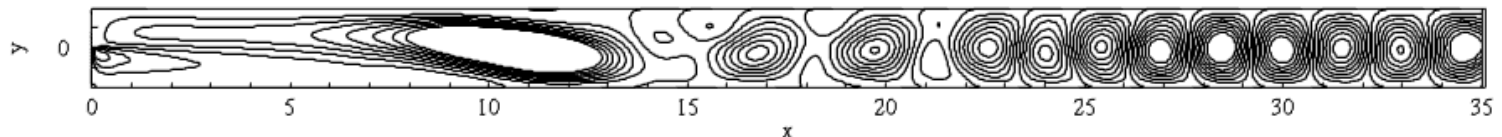

(a) Sem zona de amortecimento.

$>0$

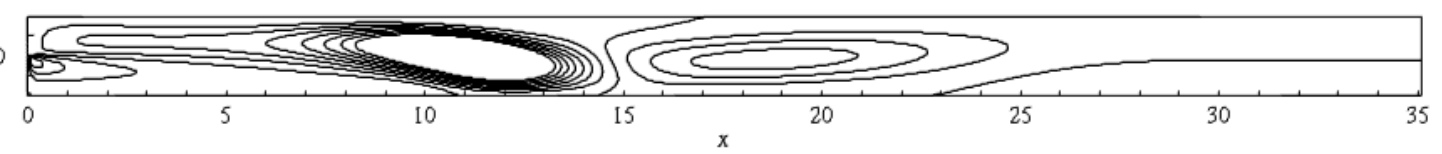

(b) Com zona de amortecimento.

Figura 3.9: Contornos de $v$ para uma expansão abrupta a $R e=600$ e $M_{\infty}=0,2$. 


\subsubsection{Paralelização}

Mesmo utilizando uma discretização espacial de alta ordem de precisão, malhas refinadas são necessárias para resolver todas as escalas em uma simulação DNS. O custo computacional para resolver este tipo de simulação em um único processador muitas vezes é proibitivo. Optouse, portanto, pela implementação de um código com capacidade de processamento paralelo.

A técnica de paralelização utilizada foi a de decomposição do domínio. Nesta técnica, o domínio é particionado e os vários subdomínios são resolvidos em processos diferentes. Para diferenças finitas explícitas basta que haja uma comunicação entre os pontos próximos às fronteiras de cada subdomínio para que seja possível calcular a aproximação para a derivada em todos os pontos. No caso de diferenças finitas compactas, todos os pontos ao longo de uma linha em uma dada direção, $x, y$, ou $z$, formam um sistema linear, cuja solução é a aproximação para a derivada.

A abordagem mais comum para a paralelização desses métodos compactos é a sobreposição de pontos nos subdomínios (MORGAN; VISBAL; RIZZETTA, 2006), de forma que a discretização fica compacta por partes e os pontos de sobreposição são atualizados explicitamente em algum momento. Este tipo de paralelização faz com que o número de pontos a serem calculados aumente, pois os pontos de sobreposição são calculados por mais de um subdomínio e reduz a precisão das diferenças finitas compactas, a menos que seja utilizado um número de pontos de sobreposição suficientemente alto.

A paralelização empregada no código DNS segue uma estratégia diferente e foi implementada através da biblioteca 2 decomp (LI; LAIZET, 2010). O domínio inicialmente é particionado em $y$ e $z$, então cada subdomínio possui todos os pontos ao longo da direção $x$ (figura 3.10(a)) e a as diferenças finitas compactas podem ser calculadas para esta direção. Em seguida, uma comunicação entre os processos, envolvendo uma operação de transposição, transforma o particionamento que preserva a direção $x$ para um particionamento que preserva a direção $y$ (figura 3.10(b)) e as diferenças finitas compactas podem ser calculadas para a direção $y$. $\mathrm{O}$ procedimento análogo é feito para obter um particionamento que preserva a direção $z$ (figura 


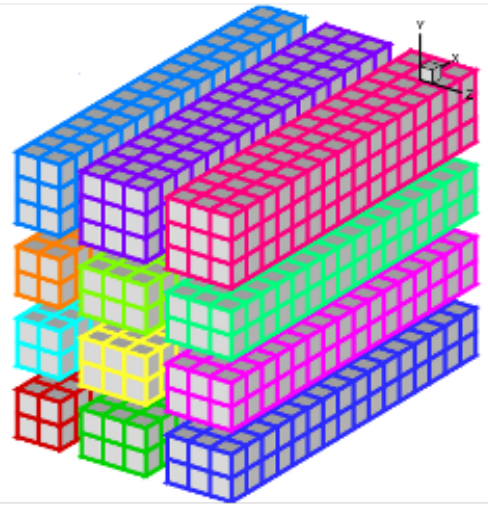

(a) Direção $x$ completa.

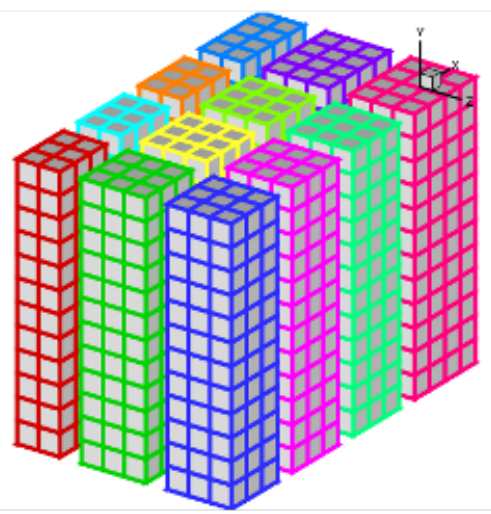

(b) Direção $y$ completa.

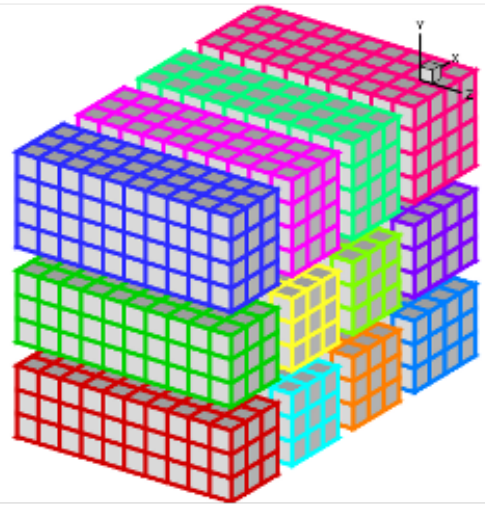

(c) Direção z completa.

Figura 3.10: Decomposição do domínio. Cada cor representa um processo.

3.10(c)) e as diferenças finitas compactas são calculadas para esta direção. Finalmente, com mais uma operação de transposição, o particionamento é levado para sua configuração inicial (figura 3.10(a)), desta vez com todas as derivadas calculadas. A equação de Navier-Stokes então pode ser integrada no tempo.

Para verificar a escalabilidade do código usou-se uma simulação DNS em uma malha 100x100x100. O speedup (figura 3.11(a)) é uma medida de quanto o código paralelo sendo calculado em $p$ processos é mais rápido que o código sequencial, ou, aproximadamente, o código paralelo executado em apenas um processo:

$$
S_{p}=\frac{T_{1}}{T_{p}}
$$

onde $T_{p}$ é o tempo levado para o calculo ser realizado em $p$ processos e $T_{1}$ é o tempo levado para o calculo ser realizado em apenas um processo.

A eficiência (figura 3.11(b)) mede a relação entre o tempo gasto na execução do código para processar os cálculos e o tempo total, sendo uma indicação de quanto tempo é gasto com comunicações:

$$
E_{p}=\frac{T_{1}}{p T_{p}}
$$




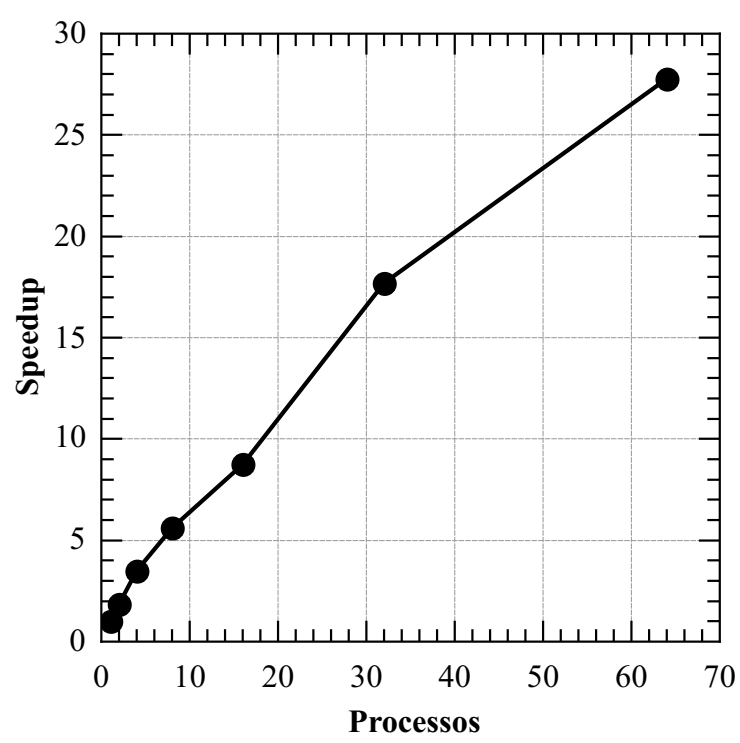

(a) Speedup.

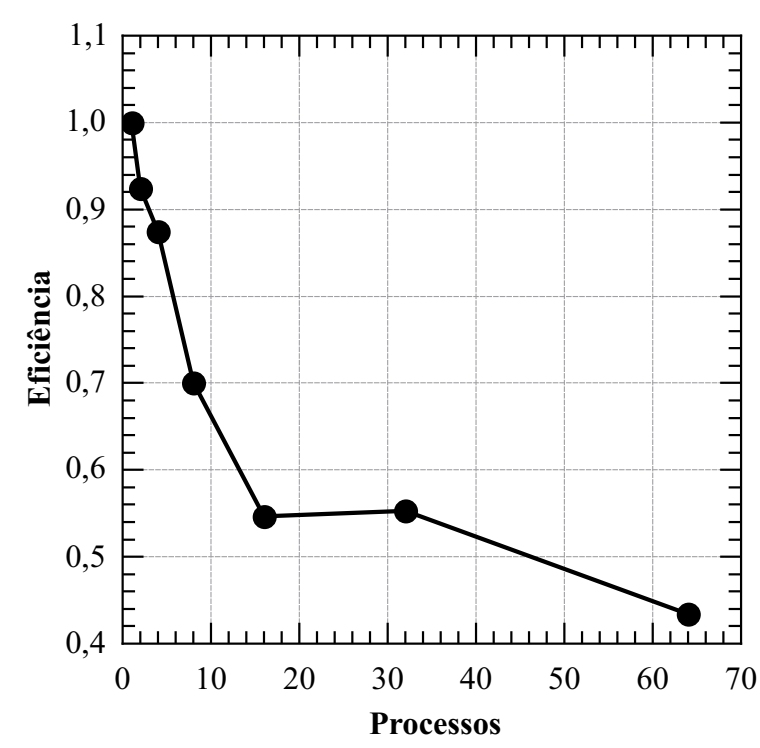

(b) Eficiência.

Figura 3.11: Escalabilidade do DNS.

\subsection{Instabilidade Hidrodinâmica Linear}

A abordagem empregada para as análises de instabilidade nesta dissertação foi a biglobal, onde a evolução de perturbações tridimensionais, homogêneas na direção $z$, sobrepostas a um escoamento base bidimensional e avaliada.

O problema de autovalor

$$
\mathscr{L} \hat{\mathbf{q}}=\omega \mathscr{R} \hat{\mathbf{q}}
$$

foi discretizado por diferenças finitas explícitas e teve sua ordem reduzida pelo algoritmo de Arnoldi antes de ser resolvido pelo método QZ.

O espectro foi calculado para diversos números de onda $\beta$ na direção $z$ em escoamentos base variando de $750 \leq R e \leq 1050$ e $0,1 \leq M_{\infty} \leq 0,9$. Os resultados foram usados para a construção das curvas neutras.

\subsubsection{Discretização Espacial}

Para a discretização espacial foi adotado o método das diferenças finitas explícitas. Embora os métodos espectrais sejam muito utilizados neste contexto devido a sua alta precisão, o pro- 


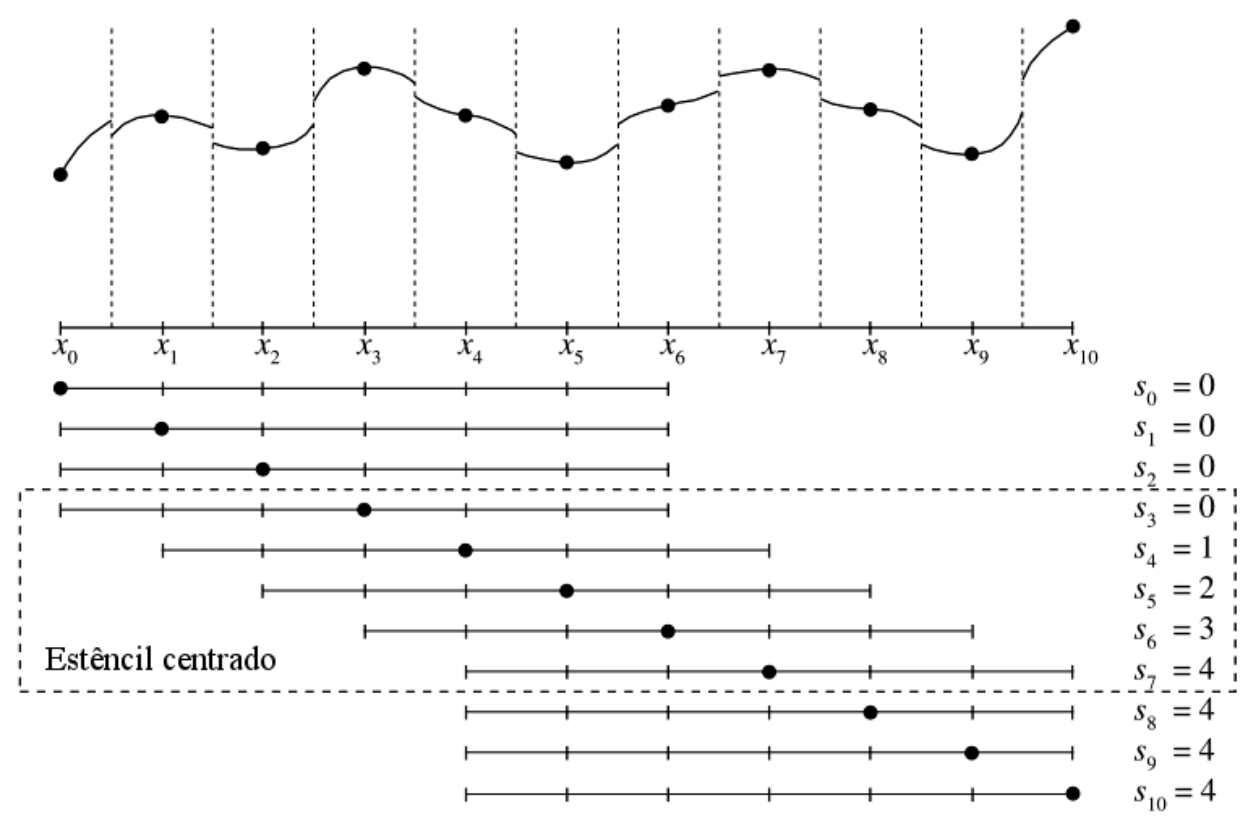

Figura 3.12: Ilustração dos estêncis das diferenças finitas. (HERMANNS, 2008).

blema de autovalor com este tipo de discretização exige grande quantidade de memória para ser resolvido computacionalmente. Se a formulação do problema é feita através de matrizes densas, os métodos espectrais são certamente superiores. No entanto, utilizando matrizes esparsas e diferenças finitas de alta ordem, um método mais eficiente é obtido (HEEG; GEURTS, 1997).

O polinômio de Lagrange de ordem $q$ que interpola uma determinada função $f=f(x)$ nos pontos $u_{j}$ é dado por:

$$
\begin{aligned}
p_{i}(x) & =\sum_{j=s_{i}}^{s_{i}+q} l_{i j}(x) u_{j}, \\
l_{i j}(x) & =\prod_{m=0}^{q} \frac{x-x_{s_{i}+m}}{x_{j}-x_{s_{i}+m}}, \text { para } s_{i}+m \neq j .
\end{aligned}
$$

Onde $s_{i}$ é o primeiro ponto utilizado para a interpolação e permite que sejam gerados polinômios interpoladores para um determinado ponto com um estêncil descentrado. O método é exemplificado na figura 3.12. No interior do domínio é usado um estêncil centrado e ao se aproximar das fronteiras são usados estêncis descentrados, mantendo o mesmo número de pontos.

A aproximação da primeira derivada de $f$ é dada analiticamente pela derivada do polinômio 
de Lagrange $p_{i}$ :

$$
\begin{aligned}
\frac{\partial f(x)}{\partial x} & \simeq \frac{\partial p_{i}(x)}{\partial x}=\sum_{j=s_{i}}^{s_{i}+q} l_{i j}^{\prime}(x) u_{j}, \\
l_{i j}^{\prime}(x) & =\sum_{m=0}^{q} \frac{1}{x_{j}-x_{s_{i}+m}} g_{i j m}^{\prime}, \text { para } i \neq j \\
g_{i j m}^{\prime} & =\prod_{n=0}^{q} \frac{x-x_{s_{i}+n}}{x_{j}-x_{s_{i}+n}}, \text { para } m \neq n
\end{aligned}
$$

Esta é uma forma fácil de se obter os coeficientes para as diferenças finitas explícitas de qualquer ordem. O método pode ser aplicado diretamente a malhas não uniformes sem a necessidade de métricas, pois a geometria já está incluída nos coeficientes. Um procedimento análogo é usado para obter a segunda derivada.

Representando os coeficientes das diferenças finitas obtidos em (3.56) na forma matricial, temos:

$$
\frac{\partial f(x)}{\partial x}=\mathscr{D}_{x}^{1 D} f
$$

Os operadores lineares $\mathscr{L}$ e $\mathscr{R}$, no entanto, são bidimensionais. As matrizes de derivação bidimensionais podem ser obtidas a partir da matriz unidimensional $\mathscr{D}^{1 D}$ através do produto de Kronecker com a matriz identidade $\mathscr{I}$ :

$$
\begin{aligned}
\mathscr{D}_{x} & =\mathscr{D}_{x}^{1 D} \otimes \mathscr{I}, \\
\mathscr{D}_{y} & =\mathscr{I} \otimes \mathscr{D}_{y}^{1 D}, \\
\mathscr{D}_{x y} & =\left(\mathscr{D}_{x}^{1 D} \otimes \mathscr{I}\right) \times\left(\mathscr{D}_{y}^{1 D} \otimes \mathscr{I}\right) .
\end{aligned}
$$

O produto de Kronecker entre uma matriz A, de ordem $m \times n$, e uma matriz $\mathbf{B}$, de ordem $p \times q$, é definido como:

$$
\mathbf{A} \otimes \mathbf{B}=\left[\begin{array}{ccc}
a_{11} \mathbf{B} & \ldots & a_{1 n} \mathbf{B} \\
\vdots & \ddots & \vdots \\
a_{m 1} \mathbf{B} & \ldots & a_{m n} \mathbf{B}
\end{array}\right]
$$




\subsubsection{Condições de Contorno}

Condições de contorno homogêneas precisam ser impostas para o problema de autovalor. Para exemplificar o procedimento utilizado será adotado um problema de autovalor unidimensional.

A condição de contorno de Dirichlet, para um ponto qualquer é obtida zerando-se as linhas das matrizes $\mathscr{L}$ e $\mathscr{R}$ correspondentes. Em seguida é atribuído o valor 1 ao elemento pertencente à diagonal da matriz $\mathscr{L}$ que corresponde ao ponto de aplicação da condição de contorno. Supondo que a condição será aplicada no ponto $\hat{q}_{1}$, o problema de autovalor assumirá a forma:

$$
\left[\begin{array}{ccccc}
1 & 0 & 0 & \ldots & 0 \\
\mathscr{L}_{2,1} & \mathscr{L}_{2,2} & \mathscr{L}_{2,3} & \ldots & \mathscr{L}_{2, n} \\
\vdots & \vdots & \vdots & \ddots & \vdots \\
\mathscr{L}_{n, 1} & \mathscr{L}_{n, 2} & \mathscr{L}_{n, 3} & \ldots & \mathscr{L}_{n, n}
\end{array}\right]\left[\begin{array}{c}
\hat{q}_{1} \\
\hat{q}_{2} \\
\vdots \\
\hat{q}_{n}
\end{array}\right]=\omega\left[\begin{array}{ccc}
0 & \ldots & 0 \\
\mathscr{R}_{2,1} & \ldots & \mathscr{R}_{2, n} \\
\vdots & \ddots & \vdots \\
\mathscr{R}_{n, 1} & \ldots & \mathscr{R}_{n, n}
\end{array}\right]\left[\begin{array}{c}
\hat{q}_{1} \\
\hat{q}_{2} \\
\vdots \\
\hat{q}_{n}
\end{array}\right]
$$

Para a condição de contorno de Neumann, ao invés de se atribuir o valor 1 ao elemento correspondente na diagonal da matriz $\mathscr{L}$, a linha toda é preenchida com os coeficientes da matriz de derivação $\mathscr{D}_{x}^{1 D}$ para a mesma posição:

$$
\left[\begin{array}{ccccc}
\mathscr{D}_{x_{1,1}}^{1 D} & \mathscr{D}_{x_{1,2}}^{1 D} & \mathscr{D}_{x_{1,3}}^{1 D} & \ldots & \mathscr{D}_{x_{1, n}}^{1 D} \\
\mathscr{L}_{2,1} & \mathscr{L}_{2,2} & \mathscr{L}_{2,3} & \ldots & \mathscr{L}_{2, n} \\
\vdots & \vdots & \vdots & \ddots & \vdots \\
\mathscr{L}_{n, 1} & \mathscr{L}_{n, 2} & \mathscr{L}_{n, 3} & \ldots & \mathscr{L}_{n, n}
\end{array}\right]\left[\begin{array}{c}
\hat{q}_{1} \\
\hat{q}_{2} \\
\vdots \\
\hat{q}_{n}
\end{array}\right]=\omega\left[\begin{array}{ccc}
0 & \ldots & 0 \\
\mathscr{R}_{2,1} & \ldots & \mathscr{R}_{2, n} \\
\vdots & \ddots & \vdots \\
\mathscr{R}_{n, 1} & \ldots & \mathscr{R}_{n, n}
\end{array}\right]\left[\begin{array}{c}
\hat{q}_{1} \\
\hat{q}_{2} \\
\vdots \\
\hat{q}_{n}
\end{array}\right]
$$

Para o problema de autovalor bidimensional, o procedimento é o mesmo. Existe apenas uma complexidade a mais para localizar as posições da matriz $\mathscr{L}$ que receberão os coeficientes da matriz de derivação bidimensional que foi obtida pelo produto de Kronecker, conforme já descrito.

No caso da pressão na parede, não existe uma condição de contorno física e a condição de compatibilidade, obtida pela manipulação do operador linear apresentado no Apêndice B, é 
imposta:

$$
\begin{aligned}
& \frac{1}{\gamma M a_{\infty}^{2}} \frac{\partial \hat{p}}{\partial x}+\frac{1}{\bar{T}}\left(\bar{u} \frac{\partial \bar{u}}{\partial x}+\bar{v} \frac{\partial \bar{u}}{\partial y}\right) \hat{p}= \\
& \frac{4}{3} \frac{\bar{\mu}}{\operatorname{Re}} \frac{\partial^{2} \hat{u}}{\partial x^{2}}+\left(\frac{4}{3} \frac{1}{\operatorname{Re}} \frac{d \bar{\mu}}{d \bar{T}} \frac{\partial \bar{T}}{\partial x}-\bar{\rho} \bar{u}\right) \frac{\partial \hat{u}}{\partial x}+\frac{\bar{\mu}}{\operatorname{Re}} \frac{\partial^{2} \hat{u}}{\partial y^{2}}+\left(\frac{1}{\operatorname{Re}} \frac{d \bar{\mu}}{d \bar{T}} \frac{\partial \bar{T}}{\partial y}-\bar{\rho} \bar{v}\right) \frac{\partial \hat{u}}{\partial y}+\frac{1}{3} \frac{\bar{\mu}}{\operatorname{Re}} \frac{\partial^{2} \hat{v}}{\partial x \partial y} \\
& +\frac{1}{\operatorname{Re}} \frac{d \bar{\mu}}{d \bar{T}} \frac{\partial \bar{T}}{\partial y} \frac{\partial \hat{v}}{\partial x}-\frac{2}{3} \frac{\bar{\mu}}{\operatorname{Re}} \frac{d \bar{\mu}}{d \bar{T}} \frac{\partial \bar{T}}{\partial x} \frac{\partial \hat{v}}{\partial y}+\frac{1}{3} i \beta \frac{\bar{\mu}}{\operatorname{Re}} \frac{\partial \hat{w}}{\partial x}+\frac{1}{\operatorname{Re}} \frac{d \bar{\mu}}{d \bar{T}}\left(\frac{4}{3} \frac{\partial \bar{u}}{\partial x}-\frac{2}{3} \frac{\partial \bar{v}}{\partial y}\right) \frac{\partial \hat{T}}{\partial x} \\
& +\frac{1}{\operatorname{Re}} \frac{d \bar{\mu}}{d \bar{T}}\left(\frac{\partial \bar{u}}{\partial y}+\frac{\partial \bar{v}}{\partial x}\right) \frac{\partial \hat{T}}{\partial y}+\left[\frac{1}{\operatorname{Re}} \frac{d \bar{\mu}}{d \bar{T}}\left(i \beta \frac{\partial \bar{w}}{\partial x}+\frac{4}{3} \frac{\partial^{2} \bar{u}}{\partial x^{2}}+\frac{\partial^{2} \bar{u}}{\partial y^{2}}+\frac{1}{3} \frac{\partial^{2} \bar{v}}{\partial x \partial y}\right)\right. \\
& \left.+\frac{1}{\operatorname{Re}} \frac{d^{2} \bar{\mu}}{d \bar{T}^{2}}\left(\frac{4}{3} \frac{\partial \bar{T}}{\partial x} \frac{\partial \bar{u}}{\partial x}+\frac{\partial \bar{T}}{\partial y} \frac{\partial \bar{u}}{\partial y}+\frac{\partial \bar{T}}{\partial y} \frac{\partial \bar{v}}{\partial x}-\frac{2}{3} \frac{\partial \bar{T}}{\partial x} \frac{\partial \bar{v}}{\partial y}\right)+\frac{\bar{\rho}}{\bar{T}}\left(\bar{u} \frac{\partial \bar{u}}{\partial x}+\bar{v} \frac{\partial \bar{u}}{\partial y}\right)\right] \hat{T} \\
& \frac{1}{\gamma M a_{\infty}^{2}} \frac{\partial \hat{p}}{\partial y}+\frac{1}{\bar{T}}\left(\bar{u} \frac{\partial \bar{v}}{\partial x}+\bar{v} \frac{\partial \bar{v}}{\partial y}\right) \hat{p}= \\
& \frac{\bar{\mu}}{\operatorname{Re}} \frac{\partial^{2} \hat{v}}{\partial x^{2}}+\left(\frac{1}{\operatorname{Re}} \frac{d \bar{\mu}}{d \bar{T}} \frac{\partial \bar{T}}{\partial x}-\bar{\rho} \bar{u}\right) \frac{\partial \hat{v}}{\partial x}+\frac{4}{3} \frac{\bar{\mu}}{\operatorname{Re}} \frac{\partial^{2} \hat{v}}{\partial y^{2}}+\left(\frac{4}{3} \frac{1}{\operatorname{Re}} \frac{d \bar{\mu}}{d \bar{T}} \frac{\partial \bar{T}}{\partial y}-\bar{\rho} \bar{v}\right) \frac{\partial \hat{v}}{\partial y}+\frac{1}{3} \frac{\bar{\mu}}{\operatorname{Re}} \frac{\partial^{2} \hat{u}}{\partial x \partial y} \\
& -\frac{2}{3} \frac{1}{\operatorname{Re}} \frac{d \bar{\mu}}{d \bar{T}} \frac{\partial \bar{T}}{\partial y} \frac{\partial \hat{u}}{\partial x}+\frac{1}{\operatorname{Re}} \frac{d \bar{\mu}}{d \bar{T}} \frac{\partial \bar{T}}{\partial x} \frac{\partial \hat{u}}{\partial y}+\frac{1}{3} i \beta \frac{\bar{\mu}}{\operatorname{Re}} \frac{\partial \hat{w}}{\partial y}+\frac{1}{\operatorname{Re}} \frac{d \bar{\mu}}{d \bar{T}}\left(\frac{\partial \bar{u}}{\partial y}+\frac{\partial \bar{v}}{\partial x}\right) \frac{\partial \hat{T}}{\partial x} \\
& +\frac{1}{R e} \frac{d \bar{\mu}}{d \bar{T}}\left(\frac{4}{3} \frac{\partial \bar{v}}{\partial y}-\frac{2}{3} \frac{\partial \bar{u}}{\partial x}\right) \frac{\partial \hat{T}}{\partial y}+\left[\frac{1}{R e} \frac{d \bar{\mu}}{d \bar{T}}\left(i \beta \frac{\partial \bar{w}}{\partial y}+\frac{4}{3} \frac{\partial^{2} \bar{v}}{\partial y^{2}}+\frac{1}{3} \frac{\partial^{2} \bar{u}}{\partial x \partial y}+\frac{\partial^{2} \bar{v}}{\partial x^{2}}\right)\right. \\
& \left.+\frac{1}{\operatorname{Re}} \frac{d^{2} \bar{\mu}}{d \bar{T}^{2}}\left(\frac{\partial \bar{T}}{\partial x} \frac{\partial \bar{u}}{\partial y}+\frac{4}{3} \frac{\partial \bar{T}}{\partial y} \frac{\partial \bar{v}}{\partial y}-\frac{2}{3} \frac{\partial \bar{T}}{\partial y} \frac{\partial \bar{u}}{\partial x}+\frac{\partial \bar{T}}{\partial x} \frac{\partial \bar{v}}{\partial x}\right)+\frac{\bar{\rho}}{\bar{T}}\left(\bar{u} \frac{\partial \bar{v}}{\partial x}+\bar{v} \frac{\partial \bar{v}}{\partial y}\right)\right] \hat{T}
\end{aligned}
$$

\subsubsection{Problema de Autovalor}

Na teoria clássica de instabilidade hidrodinâmica linear (equação de Orr-Sommerfeld), o problema de autovalor é unidimensional e representado por uma única váriavel. O tamanho das matrizes é pequeno o suficiente para que se possa aplicar o método QZ e calcular o espectro completo do problema discretizado. No caso da análise biglobal compressível, tem-se 5 variáveis primitivas e um problema de autovalor bidimensional. Considerando uma malha de tamanho $n_{x} \times n_{y}$ as matrizes obtidas teriam dimensão $n \times n$, onde $n=5 n_{x} n_{y}$. O tamanho dessas matrizes torna inviável o cálculo de todos os autovalores do problema. Através do algoritmo de Arnoldi (SAAD, 1980), faz-se uma projeção do problema de autovalor original em um subespaço de menor dimensão que contém os autovalores mais representativos.

Este método prioriza a convergência dos autovalores de maior módulo. Como os autovalo- 
res de interesse para a instabilidade hidrodinâmica são, normalmente, aqueles de menor módulo, uma transformação espectral conhecida como shift and invert é aplicada anteriormente. Esta transformação favorece a convergência dos autovalores próximos ao parâmetro $\sigma$. O problema original é reescrito na forma:

$$
\begin{aligned}
& \mathscr{A} \hat{\mathbf{q}}=\mu \hat{\mathbf{q}}, \\
& \mathscr{A}=(\mathscr{L}-\sigma \mathscr{R})^{-1} \mathscr{R}, \\
& \mu=(-i \omega-\sigma)^{-1} .
\end{aligned}
$$

O problema transformado é projetado em um subespaço de Krylov de dimensão $m$, gerado através de vetores que são multiplicações sucessivas de um vetor inicial pela matriz $\mathscr{A}$ :

$$
\mathscr{K}_{m}=\left\{\mathbf{v}, \mathscr{A} \mathbf{v}, \mathscr{A}^{2} \mathbf{v}, \ldots, \mathscr{A}^{m-1} \mathbf{v}\right\}
$$

$\mathrm{O}$ vetor inicial $\mathbf{v}$ pode ser qualquer vetor não nulo. Aqui foi adotado o vetor $\mathbf{v}=\frac{[1, \ldots, 1]}{\|[1, \ldots, 1]\|}$. Os vetores da base são ordenados em colunas em uma matriz $\mathbf{V}_{m}$. A projeção do operador $\mathscr{A}$ nesta base é dada por:

$$
\mathbf{V}_{m}^{H} \mathscr{A} \mathbf{V}_{m}=\mathbf{H}_{m},
$$

onde $\mathbf{H}_{m}$ é uma matriz de Hessenberg superior, ou seja, todos os elementos abaixo da primeira subdiagonal são nulos. O superíndice $H$ denota a matriz conjugada transposta.

O novo problema de autovalor de ordem reduzida é:

$$
\mathbf{H}_{m} \mathbf{V}_{m}^{H} \hat{\mathbf{q}}=\mu \mathbf{V}_{m}^{H} \hat{\mathbf{q}}
$$

Os autovalores de $\mathbf{H}_{m}$ são aproximações para os autovalores dominantes de $\mathscr{A}$. Se $\mathbf{y}_{i}$ é o autovetor correspondente ao autovalor $\mu_{i}$, a aproximação para o autovetor do problema original é dada pelo vetor de Ritz:

$$
\hat{\mathbf{q}}_{i}=\mathbf{V}_{m} \mathbf{y}_{i}
$$

O algoritmo de Arnoldi empregado (THEOFILIS, 2003) é mostrado a seguir: 
1. Calcular $\mathscr{B}=\mathscr{L}-\sigma \mathscr{R}$ e substituir $\mathscr{B}$ por sua decomposição LU;

2. Calcular os elementos da matrix de Hessenberg $h_{i j}$;

- Inicializar

$-\mathbf{r}_{0}=[1,1, \ldots, 1]^{T}$

$-h_{00}=\left\|\mathbf{r}_{0}\right\|$

$-\mathbf{r}_{0}=\mathbf{r}_{0} / h_{00}$

$-\mathbf{V}_{0}=\mathbf{r}_{0}$

- Para $j=0, \ldots, m-1$

$-\mathbf{r}_{j}=\mathscr{R} \mathbf{r}_{j}$

- Resolver $\mathscr{B} \mathbf{x}=\mathbf{r}_{j}$ e sobrescrever $\mathbf{r}_{j}$ com a solução de $\mathbf{x}$

- Para $i=0, \ldots, j$

$* h_{i j}=\mathbf{V}_{i} \mathbf{r}_{j}$

$* \mathbf{r}_{j}=\mathbf{r}_{j}-h_{i j} \mathbf{V}_{i}$

- $h_{j+1, j}=\left\|\mathbf{r}_{j}\right\|$

$-\mathbf{V}_{i}=\mathbf{r}_{j} / h_{j+1, j}$

3. Calcular os autovalores e autovetores de $H_{m}$ com o método QZ;

4. Calcular os vetores de Ritz $\hat{\mathbf{q}}_{i}=\mathbf{V}_{m} \mathbf{y}_{i}$.

A decomposição LU para matrizes esparsas foi calculada através da biblioteca MUMPS e a biblioteca LAPACK foi usada para o método QZ.

\subsubsection{Pseudo Espectro}

O pseudo espectro para a cavidade compressível foi calculado a partir da matriz de Hessenberg $\mathbf{H}$ obtida pelo algoritmo de Arnoldi. Como a transformação espectral shift and invert foi utilizada na formação da matriz $\mathbf{H}$, para se obter o valor da norma do resolvente em uma 
malha para $z \in \mathbb{C}$ onde se deseja obter o pseudo espectro, é necessário calcular o resolvente $\left(z^{\prime} \mathbf{I}-\mathbf{H}\right)^{-1}$ nos pontos

$$
z^{\prime}=\frac{1}{z-\sigma}
$$

onde $\sigma$ é o parametro de shift.

Em seguida, a norma do resolvente é obdita pelo inverso do maior valor singular de $\left(z^{\prime} \mathbf{I}-\right.$ H):

$$
\left\|\left(z^{\prime} \mathbf{I}-\mathbf{H}\right)^{-1}\right\|=\frac{1}{S V D_{\max }\left(\left[z^{\prime} \mathbf{I}-\mathbf{H}\right]\right)}
$$

O pseudo espectro de $\mathbf{H}$ é finalmente dado por

$$
\Lambda_{\varepsilon}(\mathbf{H})=\left\{z \in \mathbb{C}:\left\|\left(z^{\prime} \mathbf{I}-\mathbf{H}\right)^{-1}\right\| \geq \varepsilon^{-1}\right\}
$$

A norma do resolvente é calculada em uma malha no plano complexo e o pseudo espectro é mostrado pelos contornos dessa norma para $\varepsilon$ constante. 


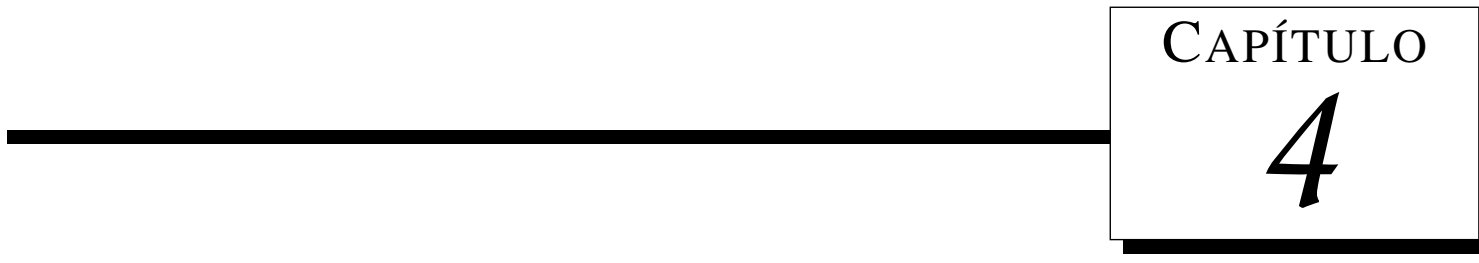

\section{Resultados}

\subsection{Equação de Helmholtz}

A equação de Helmholtz (4.1) foi usada para testar o método empregado na resolução do problema de autovalor.

$$
\nabla^{2} \hat{\phi}+\lambda^{2} \hat{\phi}=0
$$

Diferente dos outros resultados que serão apresentados, o problema de autovalor associado à equação de Helmholtz (4.1) contém apenas uma variável. Além da simplicidade, este problema possui solução analítica, permitindo uma melhor análise dos erros.

A solução análitica para este problema sujeito a condições de contorno homogêneas de Dirichlet é:

$$
\begin{aligned}
\lambda_{n, m}^{2} & =\left(\frac{n \pi}{L}\right)+\left(\frac{m \pi}{H}\right), \\
\hat{\phi}_{n, m}(x, y) & =\sin \left(\frac{n \pi x}{L}\right) \sin \left(\frac{m \pi y}{H}\right),
\end{aligned}
$$

onde $1 \leq n, m \leq \infty$. $L$ e $H$ são o tamanho do domínio em $x$ e $y$, respectivamente.

O operador $\nabla$ foi discretizado com diferenças finitas de ordens 4,8 e 12 em um domínio $L=2$ e $H=2$. A análise também foi feita com uma discretização pelo método pseudo espectral de Chebyshev-Gauss-Lobatto.

Malhas com o mesmo número de pontos em ambas as direções, variando entre 10 e 70 , 
foram usadas para verificar a convergência de malha, através do erro calculado em relação à solução analítica para o autovalor $\lambda^{2}=91,29384071007$ (figura 4.1). Não há resultado para diferenças finitas de ordem 12 e malha com 10 pontos, pois o número de pontos do domínio não é suficiente para esta ordem.

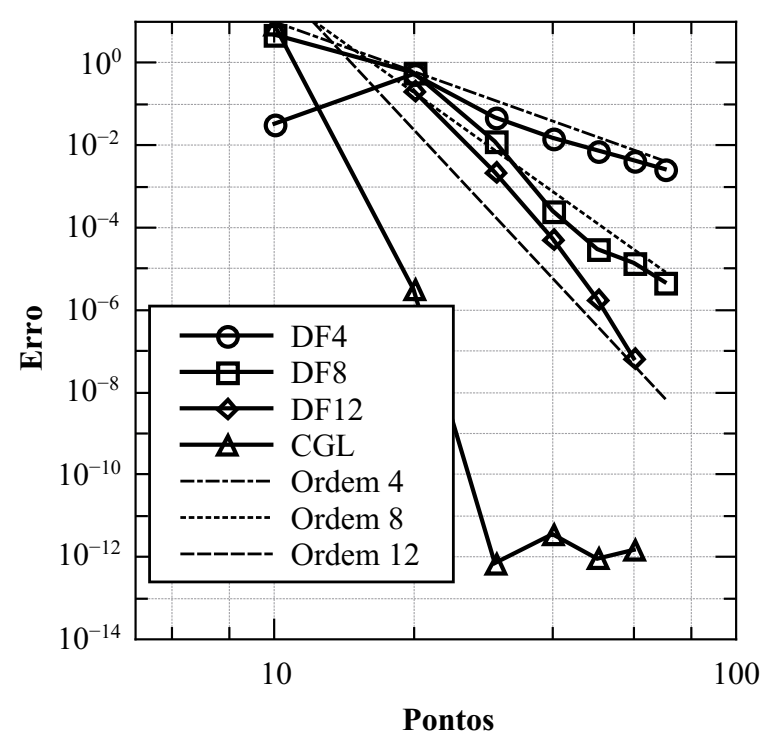

Figura 4.1: Convergência de malha para o autovalor $\lambda^{2}=91,29384071007$ da equação de Helmholtz. Discretização por diferenças finitas de ordens 4 (DF4), 8 (DF8) e 12 (DF12) e método pseudo espectral de Chebyshev-Gauss-Lobatto (CGL).

Foi escolhido um autovalor de maior módulo porque a convergência é muito rápida para os autovalores dominantes, não sendo possível observar a ordem de convergência para estes. Nesta análise, todo o espectro foi recuperado através do método QZ, para que não houvesse influência do número de vetores de Ritz utilizados no algoritmo de Arnoldi.

Utilizando o algoritmo de Arnoldi para a malha com 30 pontos e diferenças finitas de ordem 12, verifica-se que a partir de 10 vetores de Ritz já não existe diferença no resultado para o primeiro autovalor (figura 4.2).

A tabela 4.1 mostra a comparação dos quatro primeiros autovalores da equação de Helmholtz para diferenças finitas de ordem 12 e malha de 30 pontos, comparados com a solução analítica. 


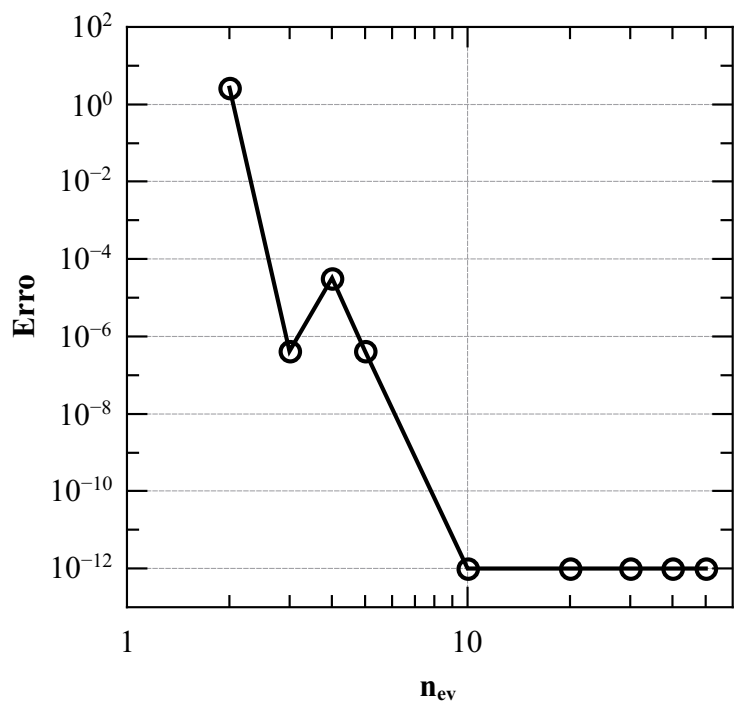

Figura 4.2: Erro para o primeiro autovalor da equação de Helmholtz para diferentes números de vetores de Ritz $n_{e v}$ com o método de Arnoldi. Diferenças finitas de ordem 12. Malha de 30 pontos.

Tabela 4.1: Quatro primeiros modos do problema de autovalor de Helmholtz.

\begin{tabular}{ccc}
\hline \hline Modo & DF12 & Analítico \\
\hline 1 & 4,934802200542 & 4,934802200545 \\
2 & 12,33700550158 & 12,33700550136 \\
3 & 19,73920880263 & 19,73920880218 \\
4 & 24,67401096957 & 24,67401100272
\end{tabular}

\subsection{Escoamento Incompressível em um Duto}

A componente $w$ da velocidade do escoamento base no interior de um duto quadrado foi obtida dado pela solução da equação de Poisson (4.4) com o termo fonte $f=-2$ e condições de contorno homogêneas de Dirichlet em toda a fronteira. As componentes $u$ e $v$ são nulas.

$$
\nabla^{2} w=f
$$

Foi adotado um domínio de $-1 \leq x \leq 1$ e $-1 \leq y \leq 1$ discretizado por uma malha uniforme e a velocidade de referência é a velocidade máxima, no centro do duto. Este problema apresenta maior complexidade e maior custo computacional que o caso anterior, a equação de Poisson, por ser um problema de autovalor bidimensional de 4 variáveis. No entanto, o escoamento base é obtido de forma simples, sem a necessidade do DNS. Dessa forma pode-se avaliar a precisão 
do método de análise de instabilidade hidrodinâmica independentemente da precisão do DNS. O escoamento base foi resolvido utilizando a mesma discretização espacial e mesma ordem de diferenças finitas usadas no problema de autovalor.

Para o caso incompressível, o problema de autovalor associado às equações de NavierStokes para as perturbações é dado por:

$$
\mathscr{L} \hat{\mathbf{q}}=\omega \mathscr{R} \hat{\mathbf{q}}
$$

onde

$$
\begin{gathered}
\mathscr{L}=\left[\begin{array}{cccc}
\mathscr{L}_{u, u} & \mathscr{L}_{u, v} & 0 & \mathscr{L}_{u, p} \\
\mathscr{L}_{v, u} & \mathscr{L}_{v, v} & 0 & \mathscr{L}_{v, p} \\
\mathscr{L}_{w, u} & \mathscr{L}_{w, v} & \mathscr{L}_{w, w} & \mathscr{L}_{w, p} \\
\mathscr{L}_{p, u} & \mathscr{L}_{p, v} & \mathscr{L}_{p, w} & 0
\end{array}\right], \\
\mathscr{R}=\left[\begin{array}{cccc}
\mathscr{R}_{u, u} & 0 & 0 & 0 \\
0 & \mathscr{R}_{v, v} & 0 & 0 \\
0 & 0 & \mathscr{R}_{w, w} & 0 \\
0 & 0 & 0 & 0
\end{array}\right] .
\end{gathered}
$$

Os elementos de $\mathscr{L}$ e $\mathscr{R}$ são dados no Apêndice A e foram retirados de Theofilis (2003). Para as componentes $\hat{u}, \hat{v}$ e $\hat{w}$, da velocidade, são aplicadas condições de contorno homogênas de Dirichlet. Para a pressao $\hat{p}$ é aplicada a condição de contorno homogênea de Neumann. As análises foram feitas para o número de onda $\beta=1$ e $R e=100$.

Para problemas desta dimensão já é significativa a diferença no custo computacional entre resolver o espectro completo e resolver parte do espectro através do algoritmo de Arnoldi. Os tempos de processamento e memória demandados na execução da análise para uma malha de $50 \times 50$ pontos com diferenças finitas de ordem 8 são apresentados na tabela 4.2.

A figura 4.3 compara os custos computacionais para resolver o autovalor dominante do duto. Foram empregadas discretizações por diferenças finitas de ordem 4, 8 e 12 e pelo método pseudo espectral de Chebyshev-Gauss-Lobatto. É possível verificar a redução tanto no uso de 
Tabela 4.2: Redução do custo computacional obtida pelo método de Arnoldi com 400 vetores de Ritz. Malha de $50 \times 50$ pontos. Diferenças finitas de ordem 8 .

\begin{tabular}{ccc}
\hline \hline & QZ completo & Arnoldi \\
\hline Tempo & $299,35 \mathrm{~s}$ & $21,39 \mathrm{~s}$ \\
Memória & $1505 \mathrm{Mb}$ & $242 \mathrm{Mb}$ \\
\hline
\end{tabular}

memória quanto no tempo de processamento proporcionada pela discretização em diferenças finitas em relação ao método pseudo espectral para atingir um mesmo erro relativo na parte imaginária do autovalor. Embora o método pseudo espectral precise de menos pontos para atingir a mesma precisão, seu custo é mais elevado. $\mathrm{Na}$ implementação foram usadas matrizes esparsas e os autovalores foram resolvidos pelo método de Arnoldi com 400 vetores de Ritz.

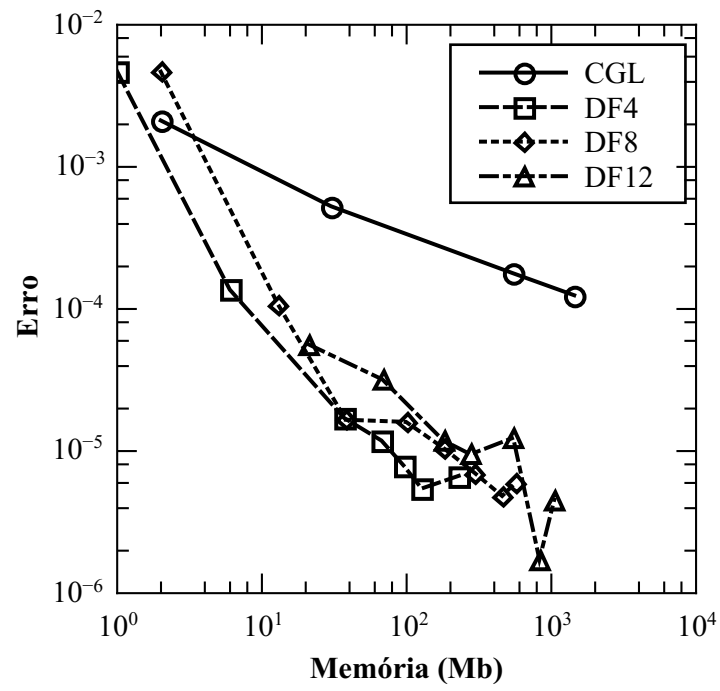

(a) Memória.

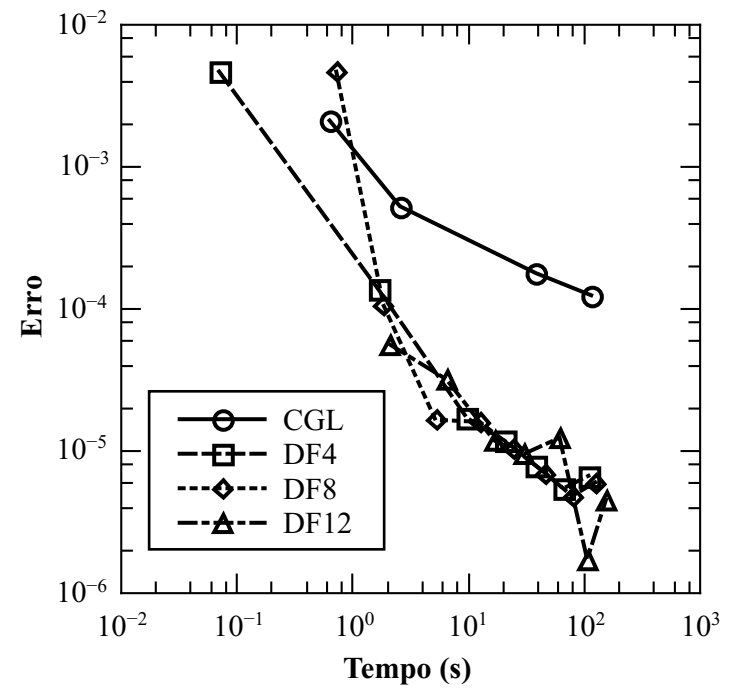

(b) Tempo computacional.

Figura 4.3: Custo computacional para o autovalor dominante do duto. $R e=100, \beta=1$. Discretização por CGL, DF4, DF8 e DF12.

A convergência de malha foi verificada para a parte imaginária do autovalor menos estável em malhas uniformes com mesma quantidade de pontos nas duas direções. Foi utilizado o método de Arnoldi com 400 vetores de Ritz e diferenças finitas de ordem 12 (figura 4.4).

O espectro para o escoamento no interior do duto é apresentado na figura 4.5 e está de acordo com os resultados apresentados por Theofilis, Duck e Owen (2004). Os quatro primeiros autovalores são dados na tabela 4.3. As respectivas autofunções são mostradas nas figuras 


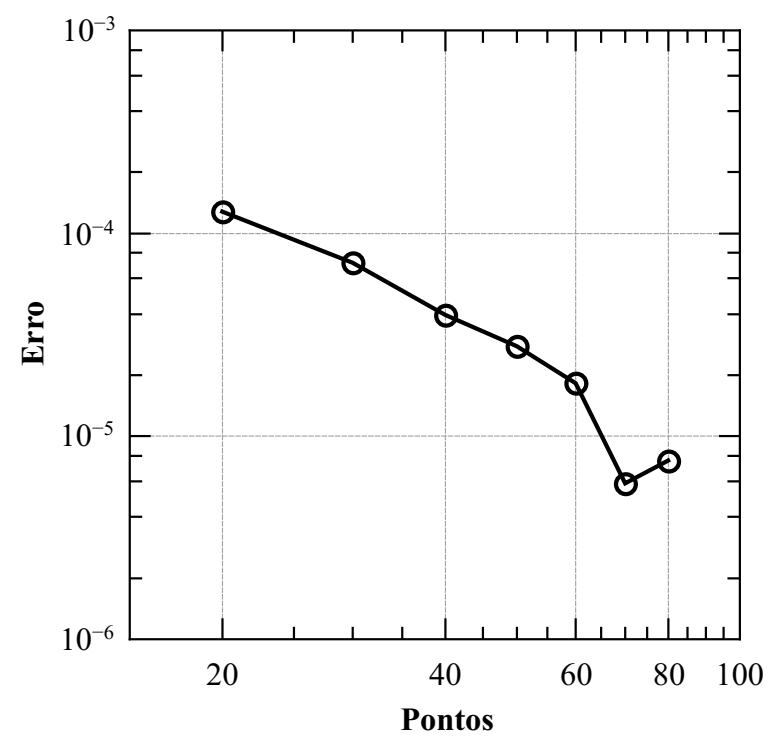

Figura 4.4: Convergência de malha a parte imaginária do autovalor dominante do escoamento incompressível no interior de um duto quadrado. $R e=100, \beta=1$.

4.6 a 4.10. O primeiro autovalor é duplo e existem duas autofunções associadas. Qualquer combinação linear dessas duas autofunções também é uma autofunção para este modo.

Tabela 4.3: Autovalores menos estáveis do duto. $R e=100, \beta=1$.

\begin{tabular}{ccc}
\hline \hline Modo & $\omega_{r}$ & $\omega_{i}$ \\
\hline $1 \mathrm{a}$ & $5.94356938 \mathrm{e}-01$ & $-1.40517720 \mathrm{e}-01$ \\
$1 \mathrm{~b}$ & $5.94356938 \mathrm{e}-01$ & $-1.40517720 \mathrm{e}-01$ \\
2 & $5.25443023 \mathrm{e}-01$ & $-2.25199981 \mathrm{e}-01$ \\
3 & $8.05922085 \mathrm{e}-01$ & $-2.44638954 \mathrm{e}-01$ \\
4 & $7.19114552 \mathrm{e}-01$ & $-2.64914891 \mathrm{e}-01$
\end{tabular}




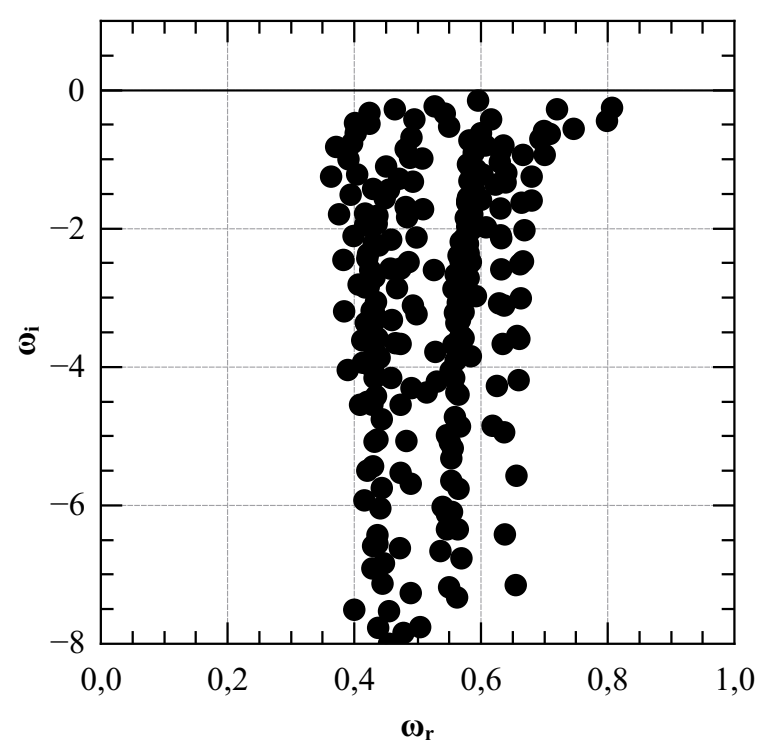

Figura 4.5: Espectro para o escoamento incompressível no interior de um duto quadrado. $R e=$ $100, \beta=1$.

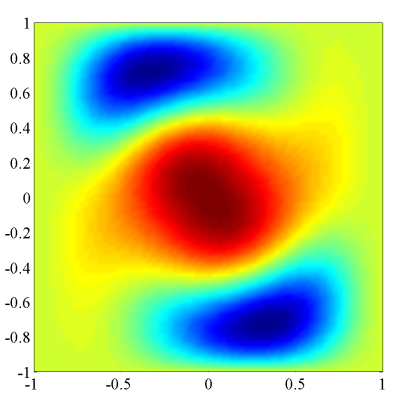

(a) $\hat{u}_{r}$.

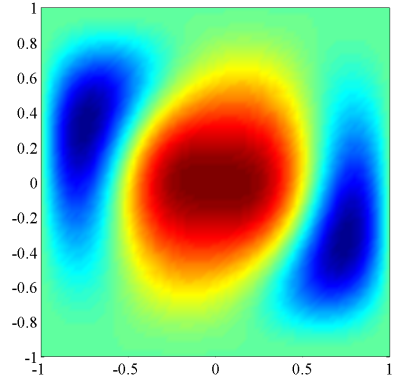

(b) $\hat{v}_{r}$.

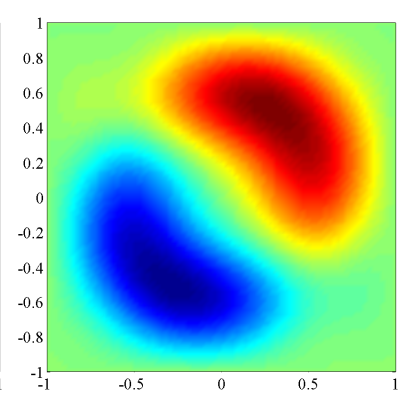

(c) $\hat{w}_{r}$.

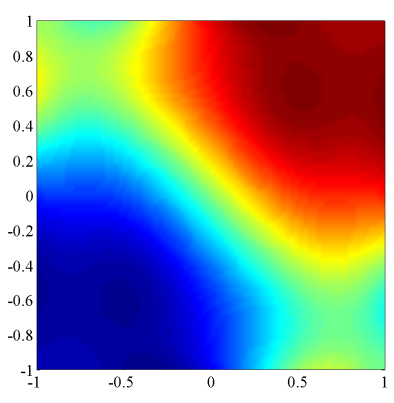

(d) $\hat{p}_{r}$.

Figura 4.6: Primeiro modo do duto (autofunção 1a). $R e=100, \beta=1$.

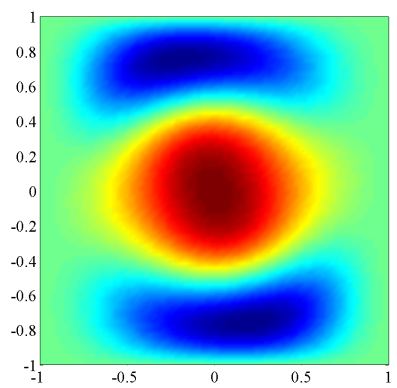

(a) $\hat{u}_{r}$.

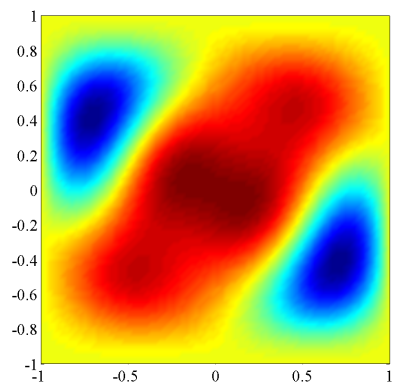

(b) $\hat{v}_{r}$.

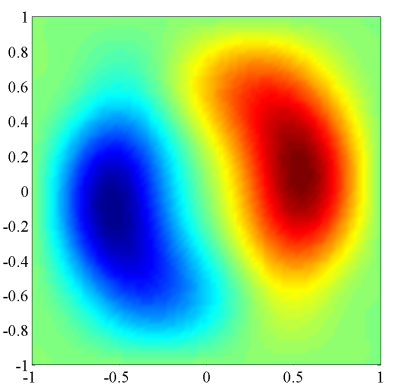

(c) $\hat{w}_{r}$.

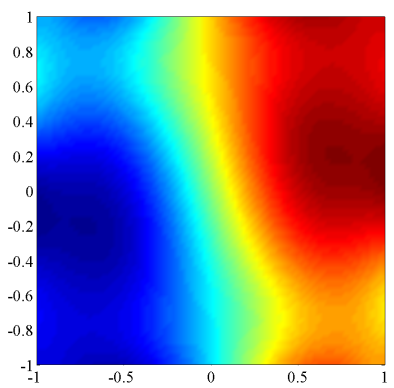

(d) $\hat{p}_{r}$.

Figura 4.7: Primeiro modo do duto (autofunção $1 \mathrm{~b}$ ). $R e=100, \beta=1$. 


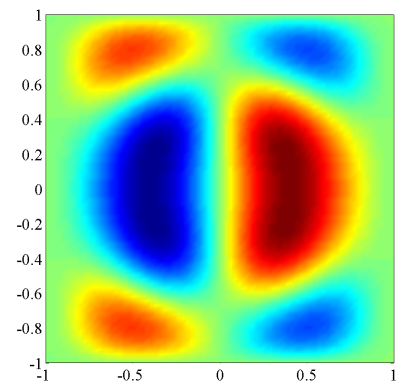

(a) $\hat{u}_{r}$.

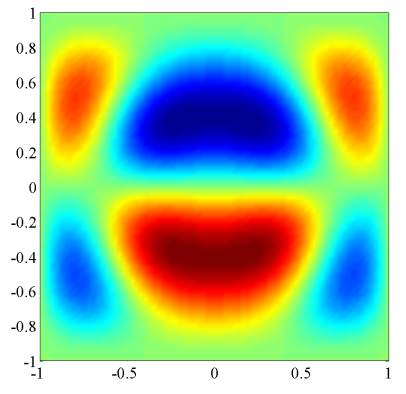

(b) $\hat{v}_{r}$.

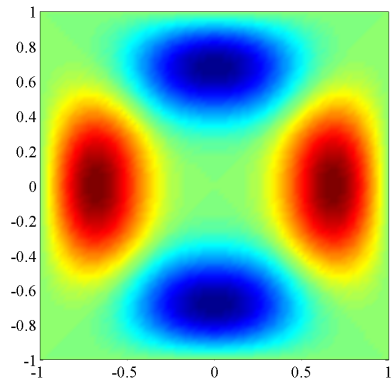

(c) $\hat{w}_{r}$.

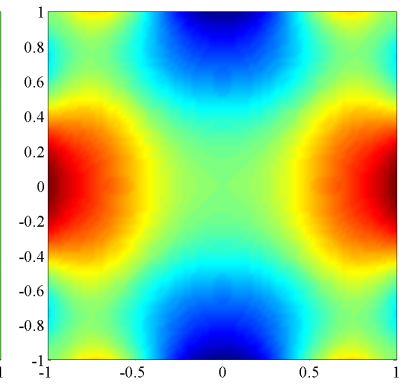

(d) $\hat{p}_{r}$.

Figura 4.8: Segundo modo do duto. $\operatorname{Re}=100, \beta=1$.

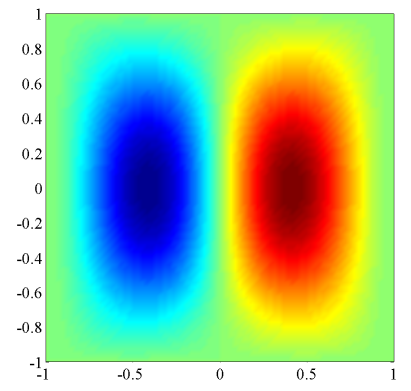

(a) $\hat{u}_{r}$.

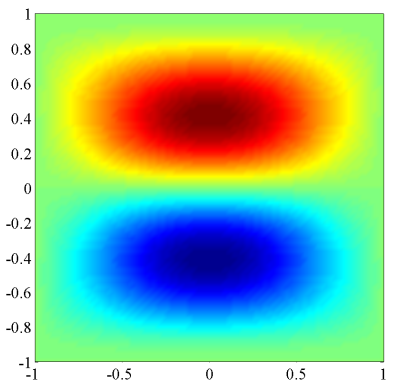

(b) $\hat{v}_{r}$.

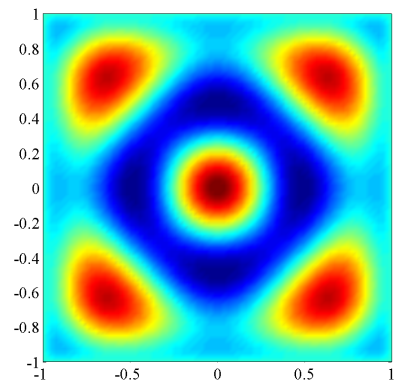

(c) $\hat{w}_{r}$.

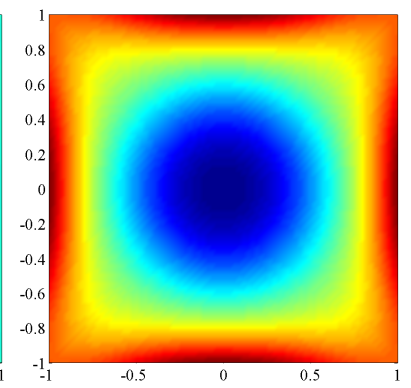

(d) $\hat{p}_{r}$.

Figura 4.9: Terceiro modo do duto. $\operatorname{Re}=100, \beta=1$.

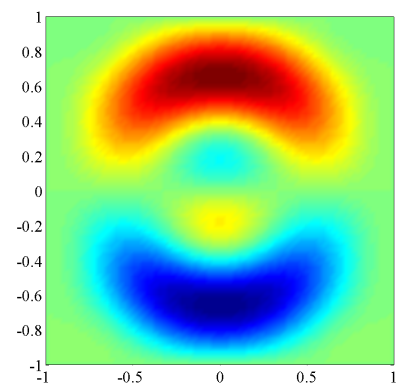

(a) $\hat{u}_{r}$.

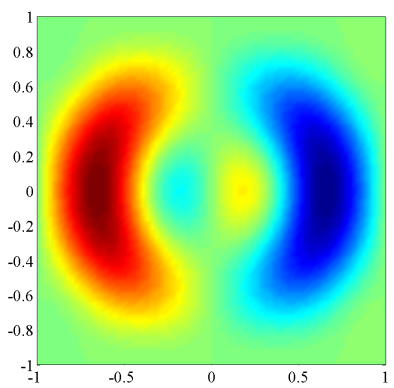

(b) $\hat{v}_{r}$.

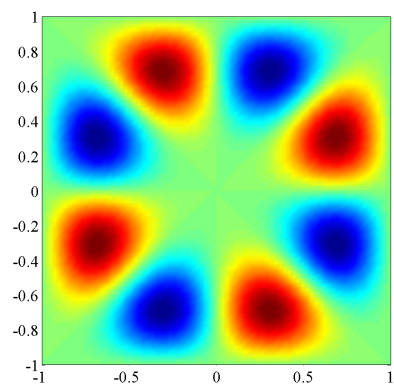

(c) $\hat{w}_{r}$.

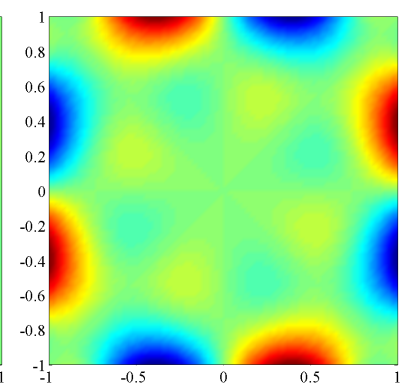

(d) $\hat{p}_{r}$.

Figura 4.10: Quarto modo do duto. $R e=100, \beta=1$. 


\subsection{Cavidade Compressível}

\subsubsection{Escoamento Base}

O escoamento base para a cavidade quadrada com tampa deslizante (figura 4.11) foi obtido através do código DNS para um domínio de tamanho $L_{x}=1, L_{y}=1$. A condição de não escorregamento $u=v=0$ foi aplicada nas paredes, junto com a condição de compatibilidade para a pressão (3.28). Para a temperatura, foi aplicada a condição de contorno isotérmica $e=$ $\frac{1}{\left(\gamma^{2}-\gamma\right) M_{\infty}^{2}}$. Para velocidade da tampa deslizante, em $y=1$, foi adotada uma condição de contorno regularizada $u=\left[1-(2 x-1)^{18}\right]^{2}$, mostrada na figura 4.12 .

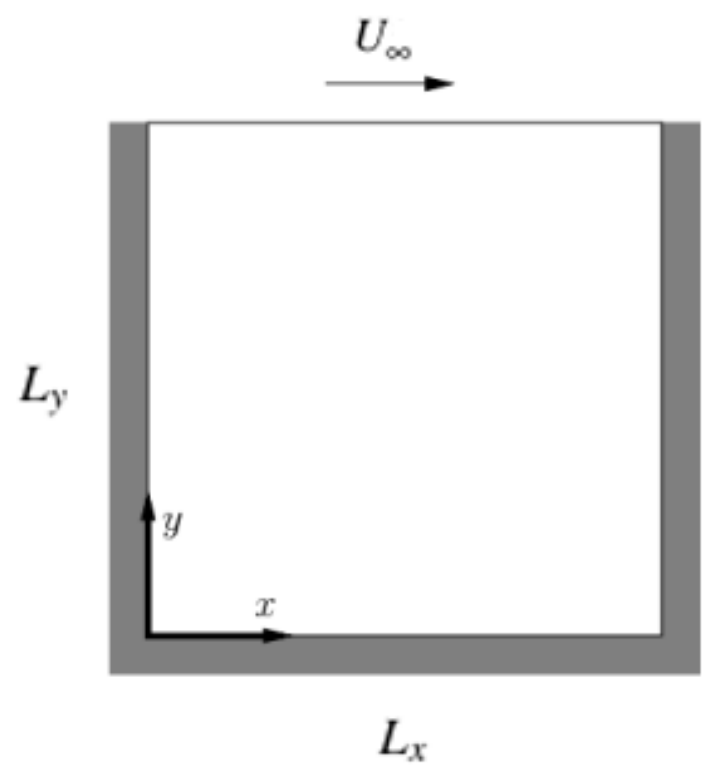

Figura 4.11: Geometria da cavidade com tampa deslizante.

Com a condição de contorno regularizada elimina-se a singularidade que ocorre nas quinas da tampa, mantendo-se a velocidade contínua em todo o contorno da fronteira a fim de facilitar a convergência. A condição de contorno regularizada foi adotada, no entanto, o código é capaz de trabalhar com velocidade uniforme na tampa. A velocidade de referênca é a velocidade da tampa, no caso uniforme, e a velocidade máxima da tampa, no caso da condição regularizada.

Observou-se que ao longo da simulação, a densidade no interior da cavidade não permanecia constante, variando com uma taxa constante após um transiente inicial. Jacobs, Kopriva e Mashayek (2005) relatam o mesmo problema, relacionado à formulação da condição de con- 


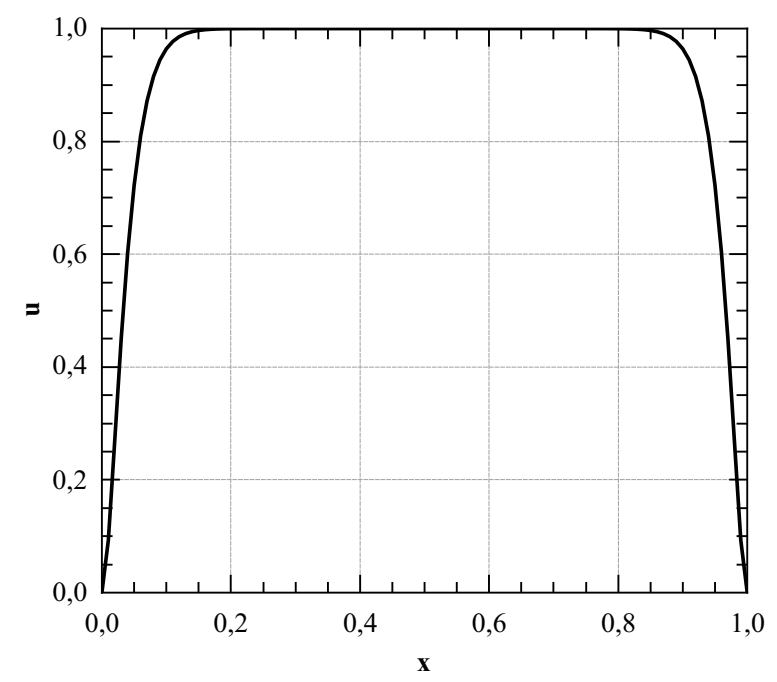

Figura 4.12: Condição de contorno regularizada para a tampa deslizante.

torno isotérmica que não é capaz de garantir fluxo de massa zero ao longo da parede e propõe uma manipulação no Osher solver que elimina este problema. Este procedimento não foi implementado. A solução adotada foi refinar a malha o suficiente para reduzir esta variação a um níve aceitavel, que não contaminasse a solução. Uma malha uniforme de $400 \times 400$ pontos foi adotada nas simulações. A convergência pode ser verificada na figura 4.13.

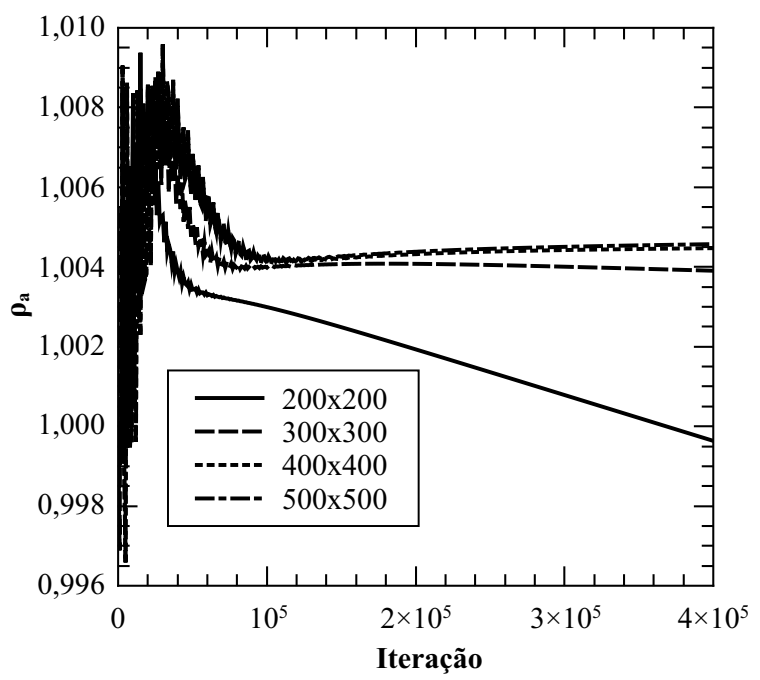

Figura 4.13: Convergência de malha para a variação de densidade no ponto $x=0,25 ; y=0,25$, no interior da cavidade. $R e=1000, M_{\infty}=0,5$.

As posições dos centros dos vórtices na cavidade para $R e=400$ e $M_{\infty}=0,1$, localizados pela interseção das linhas $u=0$ e $v=0$ (figura 4.14), foram comparadas com os resultados para o escoamento incompressível de Ghia, Ghia e Shin (1982) e Shen (1991) na tabela 4.4. O 
primeiro resultado para escoamento incompressível utiliza condição de contorno regularizada para a tampa deslizante, enquanto o segundo utiliza condição de contorno uniforme. Existe uma boa concordância entre os resultados. Na verdade, as posições dos vórtices convergem para malhas menos refinadas e a variação da densidade no interior da cavidade é o que determina a resolução apropriada.

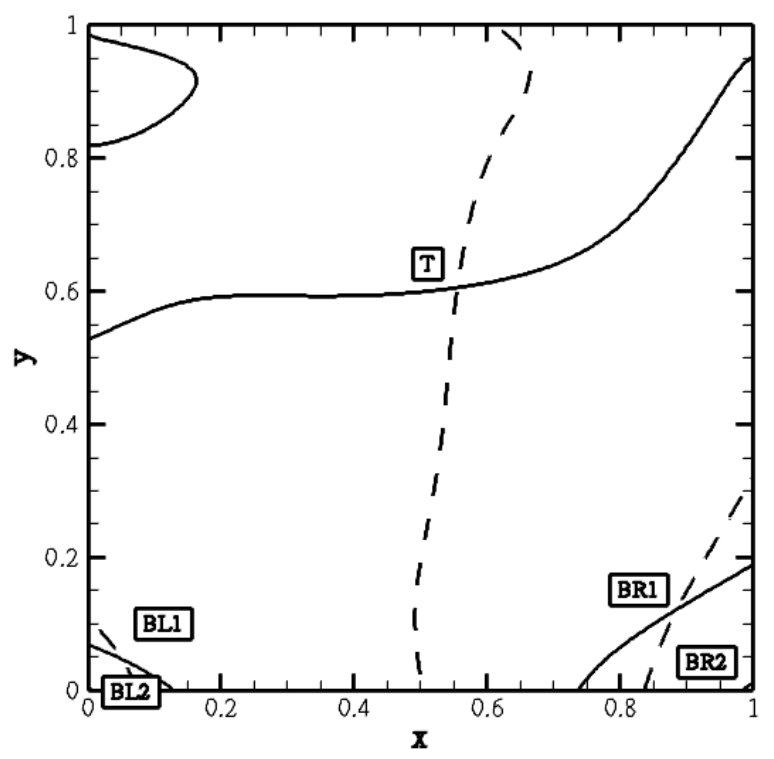

Figura 4.14: Posição dos vórtices na cavidade. $R e=1000, M_{\infty}=0,5$. Contornos para $u=0$ $\mathrm{e} v=0---$

Tabela 4.4: Posição dos vórtices na cavidade para $R e=400, M_{\infty}=0,1$. Comparação com casos incompressíveis.

\begin{tabular}{ccccc}
\hline \hline Vórtice & Posição & DNS & Ghia 1982 & Shen 1991 \\
\hline T & $x$ & 0,5553 & 0,5547 & 0,578 \\
& $y$ & 0,6055 & 0,6055 & 0,625 \\
BL1 & $x$ & 0,0510 & 0,0508 & 0,031 \\
& $y$ & 0,0472 & 0,0469 & 0,047 \\
BR1 & $x$ & 0,8856 & 0,8906 & 0,922 \\
& $y$ & 0,1223 & 0,1250 & 0,094 \\
BL2 & $x$ & 0,0029 & 0,0039 & - \\
& $y$ & 0,0029 & 0,0039 & - \\
BR2 & $x$ & 0,9930 & 0,9922 & - \\
& $y$ & 0,0070 & 0,0078 & - \\
\hline
\end{tabular}

Para as análises de instabilidade, foram gerados escoamentos base com $750 \leq R e \leq 1050$ variando em passos de 50 e $M_{\infty}$ de 0,$1 ; 0,5$ e 0,9 . O escomento base para $M a=0,5$ e $R e=1000$ 
é mostrado na figura 4.15. A compressibilidade exerce pouco efeito sobre o escoamento base na cavidade, por isso os demais escoamentos serão omitidos. De fato, (SHAH et al., 2007), que estuda o efeito do número de Mach no escoamento estacionário de uma cavidade de razão de aspecto 2 com duas tampas deslizantes, mostra que não há efeito sensível da compressibilidade nas componentes da velocidade $u$ e $v$, ocorrendo uma leve distorção nos perfis de densidade e temperatura (inferior a $1 \%$ ) entre os casos para $M_{\infty}=0,1$ e $M_{\infty}=0,4$.
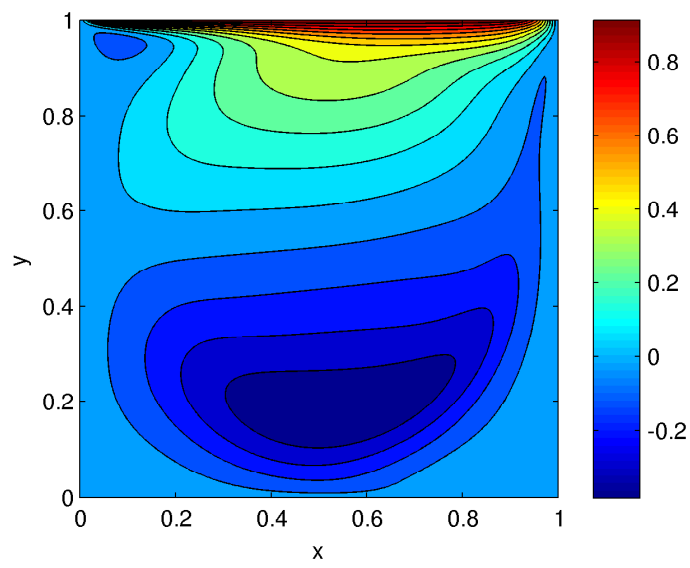

(a) $u$.

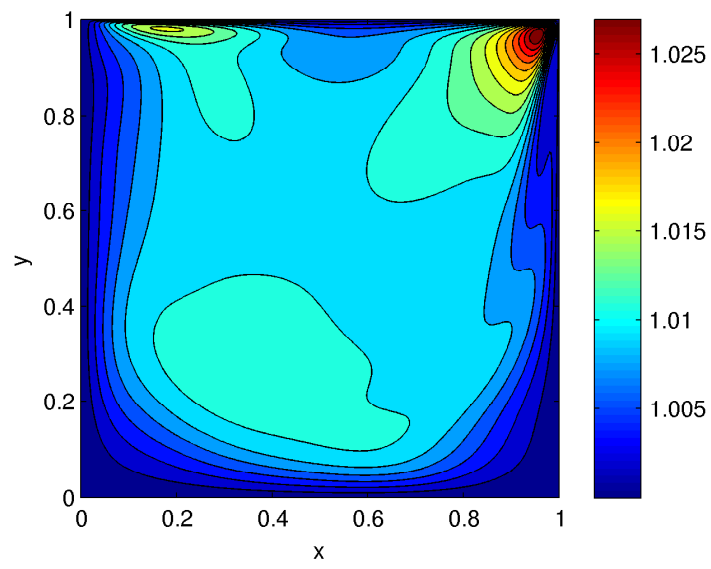

(c) $T$.

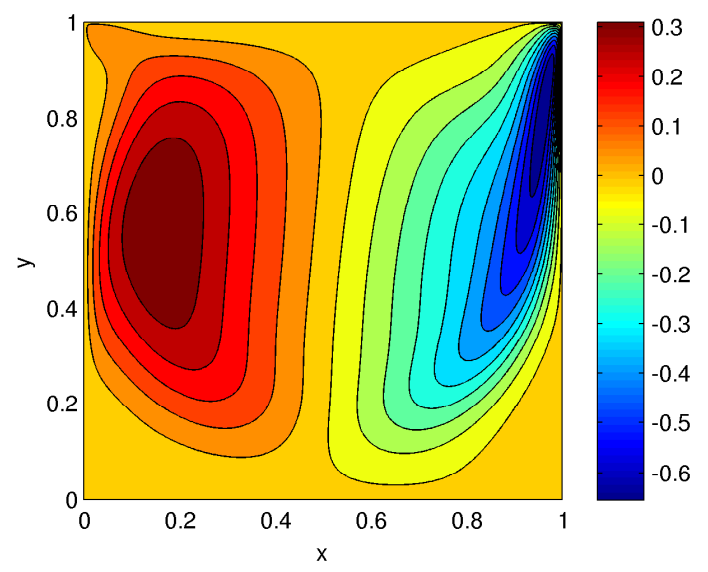

(b) $v$.

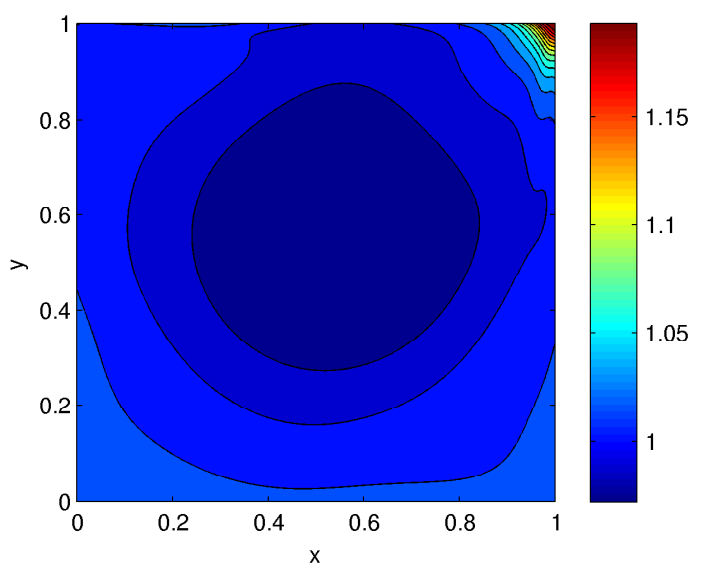

(d) $\rho$.

Figura 4.15: Escoamento base compressível para a cavidade. $R e=1000, M_{\infty}=0,5$. 


\subsubsection{Instabilidade Linear}

As análises de instabilidade hidrodinâmica foram realizadas para os escoamentos base obtidos por DNS com Reynolds variando entre 750 e 1050 e Mach entre 0,1 e 0,9.

A malha do DNS foi interpolada para uma malha de menor resolução e refinada próximo as fronteiras. O estiramento utilizado foi

$$
x=\frac{1}{2}\left[1+\frac{\tanh \left(\gamma_{s} \eta\right)}{\tanh \left(\gamma_{s}\right)}\right]
$$

onde $-1 \leq \eta \leq 1$ e $\gamma_{s}=1,4$

Os resultados para a instabilidade a Mach 0,1 são comparados aos resultados do escoamento incompressível equivalente presentes na literatura. Em seguida, a influência do número de Mach é verificada nos modos conhecidos do caso incompressível através das curvas neutras para os diferentes casos. Finalmente, novos modos encontrados, que não são observados na análise incompressível são apresentados e discutidos.

As perturbações, representadas pelas soluções do problema de autovalor (4.5) estão sujeitas às seguintes condições de contorno em toda a fronteira do domínio:

- Condição de Dirichlet homogênea para as componentes da velocidade: $\hat{u}=\hat{v}=\hat{w}=0$;

- Condição de Dirichlet homogênea para a temperatura: $\hat{T}=0$;

- Condição de compatibilidade para a pressão, conforme (3.66).

Frequentemente observa-se na literatura a imposição da condição de Neumann homogênea para a perturbação da pressão nas paredes $\left(\frac{\partial p}{\partial n}=0\right.$, onde $n$ é a direção normal à parede). Esta aproximação é válida caso a derivada segunda da componente de velocidade paralela à parede na direção normal à parede seja aproximadamente nula. Este é o caso da camada limite sobre uma placa plana. No caso da cavidade, existem regiões próximas à tampa onde não é possível usar esta aproximação (figura 4.16).

No caso da análise biglobal para um escoamento compressível, temos um problema de 


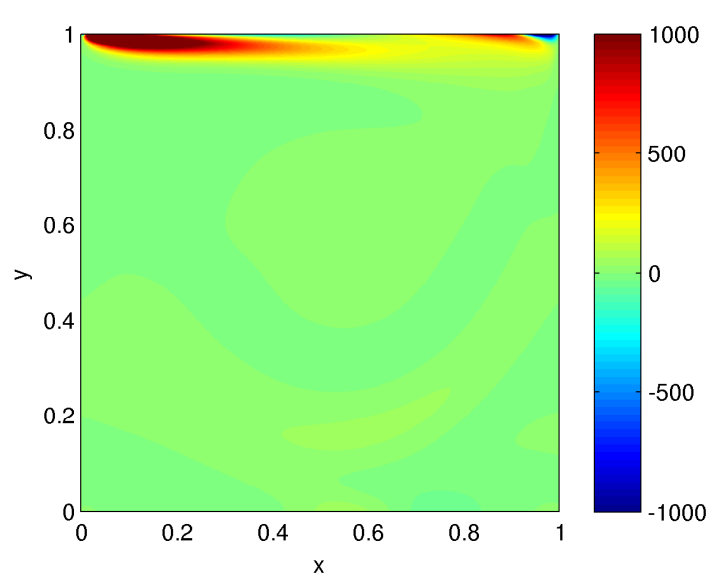

(a) $\frac{\partial^{2} \bar{u}}{\partial y^{2}}$.

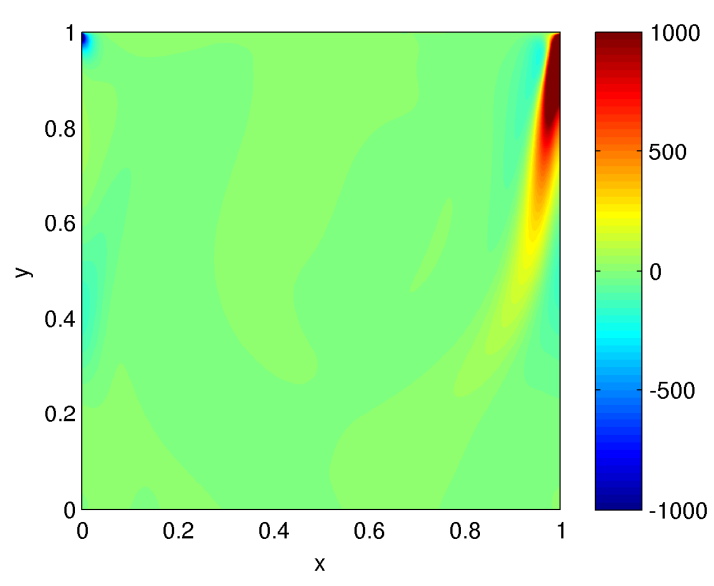

(b) $\frac{\partial^{2} \bar{v}}{\partial x^{2}}$.

Figura 4.16: Derivada segunda das componentes da velocidade no escoamento base da cavidade. $R e=1000, M_{\infty}=0,5$.

autovalor bidimensional para 5 variáveis. Fica evidente a redução de memória para o uso de diferenças finitas de alta ordem (figura 4.17) em comparação com os métodos espectrais (figura 4.18). O operador $\mathscr{L}$, discretizado com diferenças finitas de ordem 12 , possui $6 \%$ do tamanho obtido com a discretização pelo método pseudo espectral de Chebyshev-Gauss-Lobatto.

Um teste de convergência de malha mostra que para a discretização por diferenças finitas de ordem 12 uma malha de $70 \times 70$ pontos é suficiente para uma precisão de $10^{-4}$, como mostrado na tabela 4.5.

Tabela 4.5: Convergência para o primeiro modo da cavidade compressível. $R e=1000, M_{\infty}=$ $0,5, \beta=15$.

\begin{tabular}{cc}
\hline \hline Pontos & $\omega_{i}$ \\
\hline 40 & 0,11477 \\
50 & 0,10977 \\
60 & 0,10943 \\
70 & 0,10944 \\
80 & 0,10941 \\
\hline
\end{tabular}




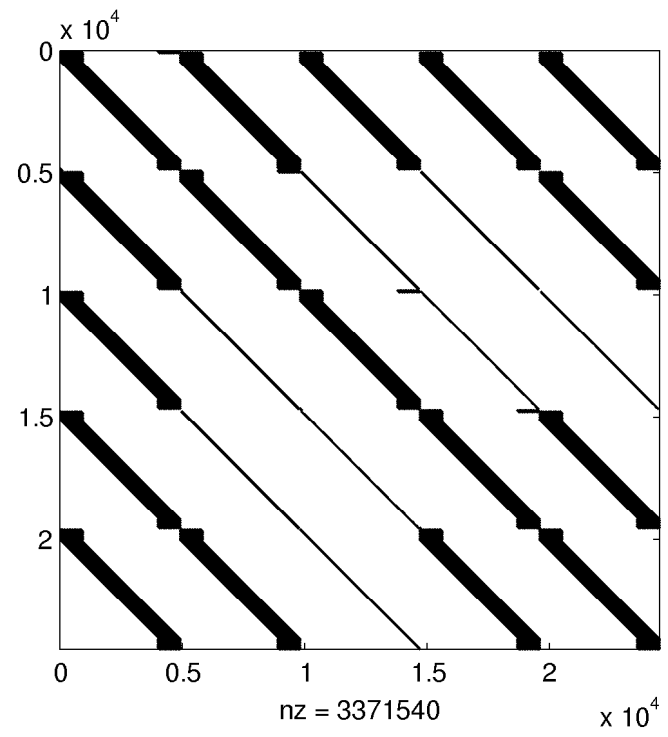

(a) $\mathscr{L}$.

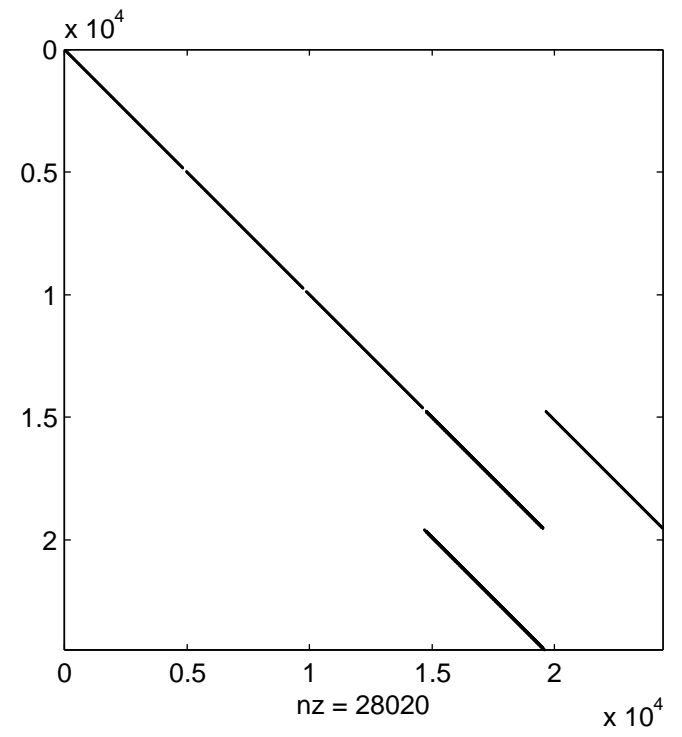

(b) $\mathscr{R}$.

Figura 4.17: Esparsidade dos operadores lineares para a cavidade compressível discretizados com diferenças finitas de ordem 12.

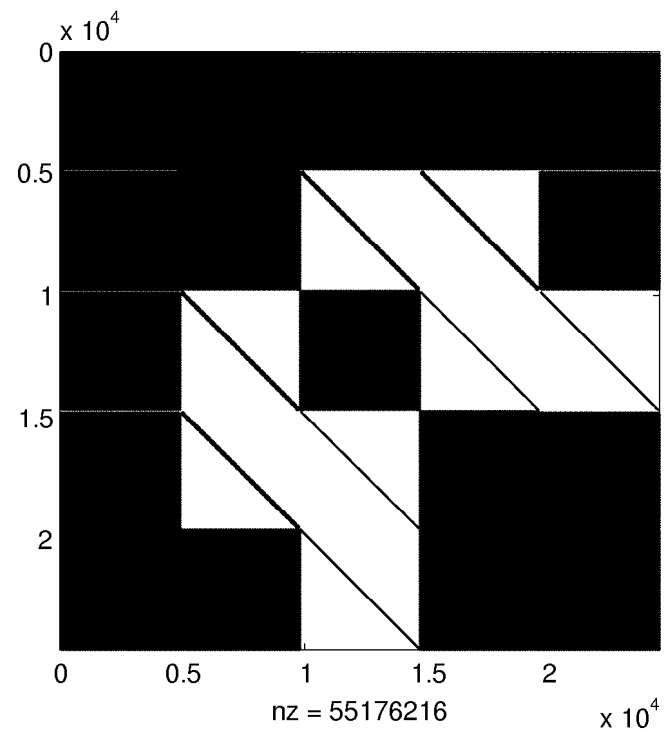

(a) $\mathscr{L}$.

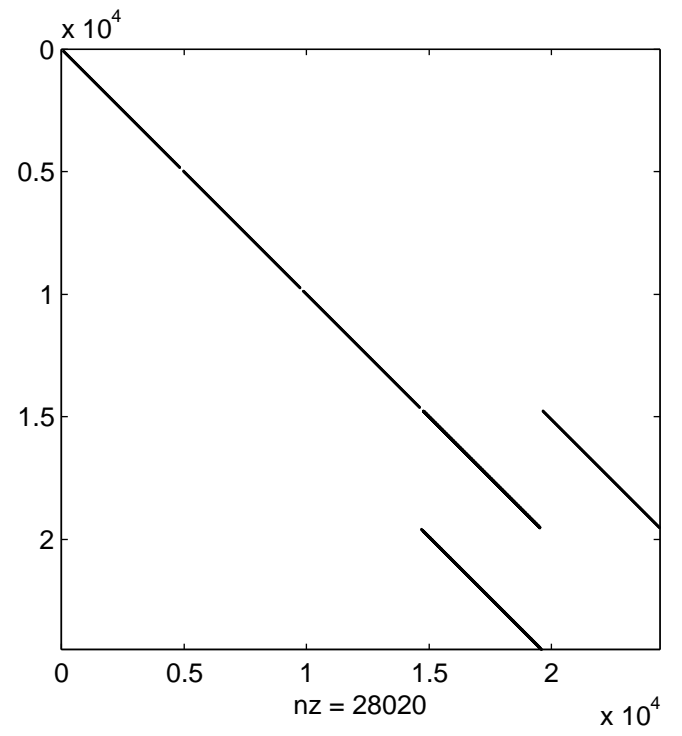

(b) $\mathscr{R}$.

Figura 4.18: Esparsidade dos operadores lineares para a cavidade compressível discretizados pelo método pseudo espectral de Chebyshev-Gauss-Lobatto. 


\section{Comparação com o Escoamento Incompressível}

Uma comparação entre o resultado para os modos dominantes da cavidade a Mach 0,1 e o resultado incompressível apresentado em (THEOFILIS; DUCK; OWEN, 2004) é apresentada na tabela 4.6. A tablea também contém o resultado obtido através da resolução do problema de autovalor incompressível associado, porém usando o escoamento base a Mach 0,1. O resultado incompressível de Theofilis, Duck e Owen (2004) foi obtido com condições de compatibilidade para a pressão e velocidade uniforme na tampa deslizante. Na tabela também são apresentadas algumas combinações entre as condições de contorno para verificar a influência nos autovalores. Dadas as diferenças relacionadas a compressibilidade e condição de contorno na tampa, os resultados estão em concordância com os encontrados na literatura. Estas diferentes combinações de condições de contorno foram feitas apenas para a comparação com o caso incompressível devido às diferenças encontradas na literatura. Para as análises compressíveis foi usada condição de contorno de compatibilidade para a pressão e condição regularizada para a velocidade da tampa. 


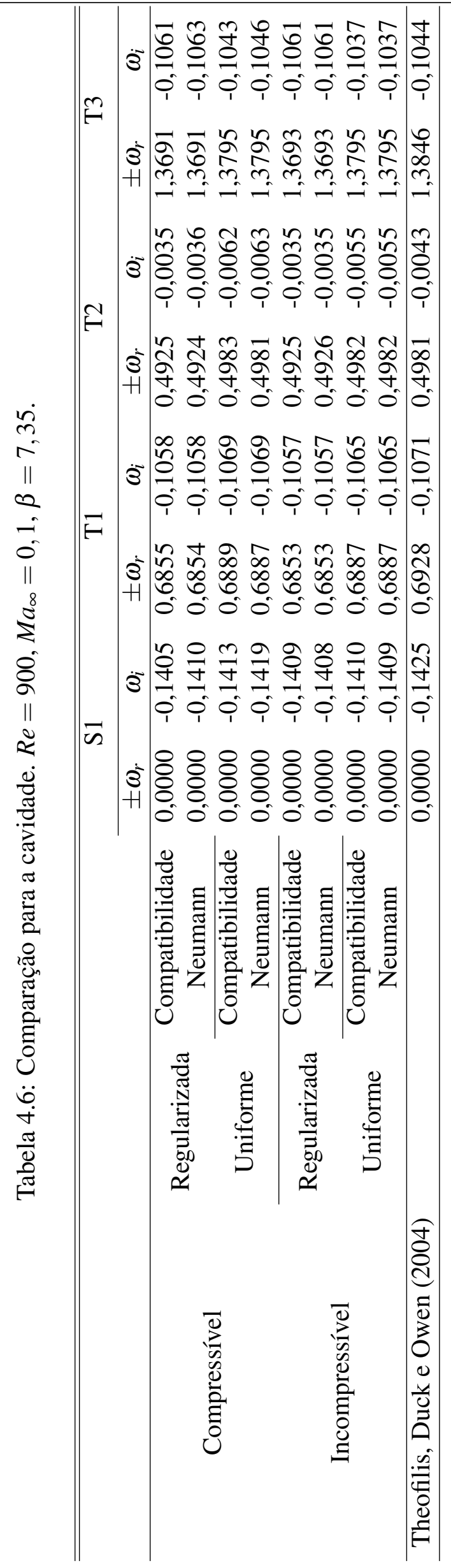




\section{Efeito do Número de Mach}

Os escoamentos base obtidos foram usados para realizar análises de instabilidade linear para números de onda $\beta$ entre 1 e 27. Para cada modo obtém-se um conjunto de autovalores em função de Renolds e Mach, $\omega=\omega(\operatorname{Re}, \beta)$. O contorno onde a parte imaginária do autovalor é nula, $\omega_{i}(\operatorname{Re}, \beta)=0$, é a chamada curva neutra e representa o limite a partir do qual o modo se torna instável. Este contorno de $\omega_{i}=0$ foi obtido através da interpolação dos casos avaliados. As curvas neutras para os diferentes números de Mach são apresentadas na figura 4.19. Os pontos representam os autovalores com parte imaginária nula para os números de Reynolds que foram de fato calculados.

Os números de Reynolds críticos e números de onda críticos são apresentados na tabela 4.7. Para os modos que também são encontrados no escoamento incompressível a compressibilidade teve sempre um efeito estabilizante, aumentando o Reynolds crítico. A ordem de dominância dos modos não foi alterada pela compressibilidade, ou seja, a sequência do mais instável (ou menos estável) ao menos instável (ou mais estável) permaneceu a mesma: S1, T1, T2, T3. Os números de onda críticos variam pouco com o número de Mach. As autofunções para os 4 modos dominantes são mostradas nas figuras 4.20 a 4.23 .

Tabela 4.7: Parâmetros críticos para a cavidade compressível.

\begin{tabular}{ccccccccc}
\hline \hline & \multicolumn{2}{c}{$\mathrm{S} 1$} & \multicolumn{2}{c}{$\mathrm{T} 1$} & \multicolumn{2}{c}{$\mathrm{T} 2$} & \multicolumn{2}{c}{$\mathrm{T} 3$} \\
\cline { 2 - 9 } & $\operatorname{Re}_{c}$ & $\beta_{c}$ & $\operatorname{Re}_{c}$ & $\beta_{c}$ & $\operatorname{Re}_{c}$ & $\beta_{c}$ & $\operatorname{Re}_{c}$ & $\beta_{c}$ \\
\hline$M_{\infty}=0,1$ & 802,98 & 15,537 & 867,92 & 16,032 & 918,67 & 7,4215 & 993,23 & 14,405 \\
$M_{\infty}=0,5$ & 812,32 & 15,604 & 877,40 & 16,149 & 924,73 & 7,4069 & 1002,0 & 14,502 \\
$M_{\infty}=0,9$ & 830,75 & 15,819 & 896,38 & 16,309 & 942,10 & 7,4040 & 1022,0 & 14,731 \\
\hline
\end{tabular}




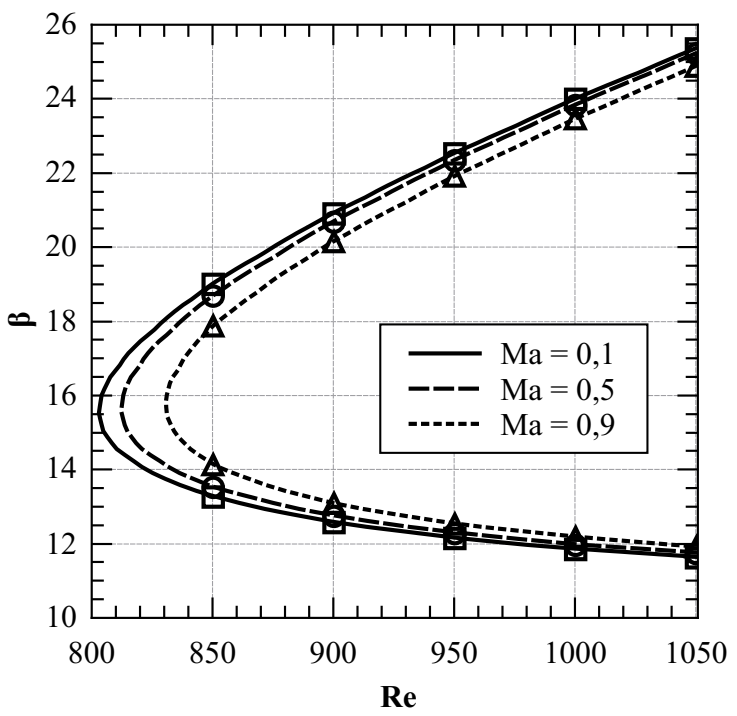

(a) Modo S1.

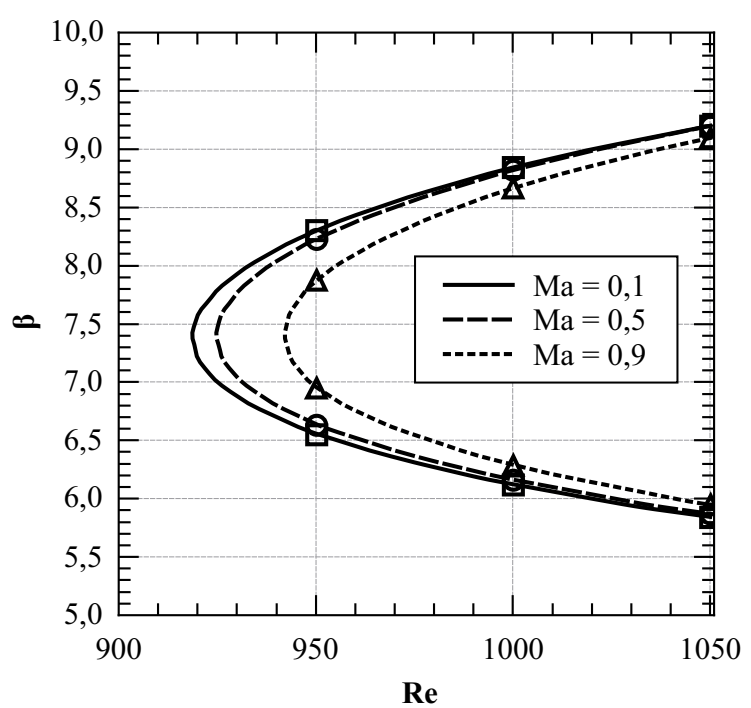

(c) Modo T2.

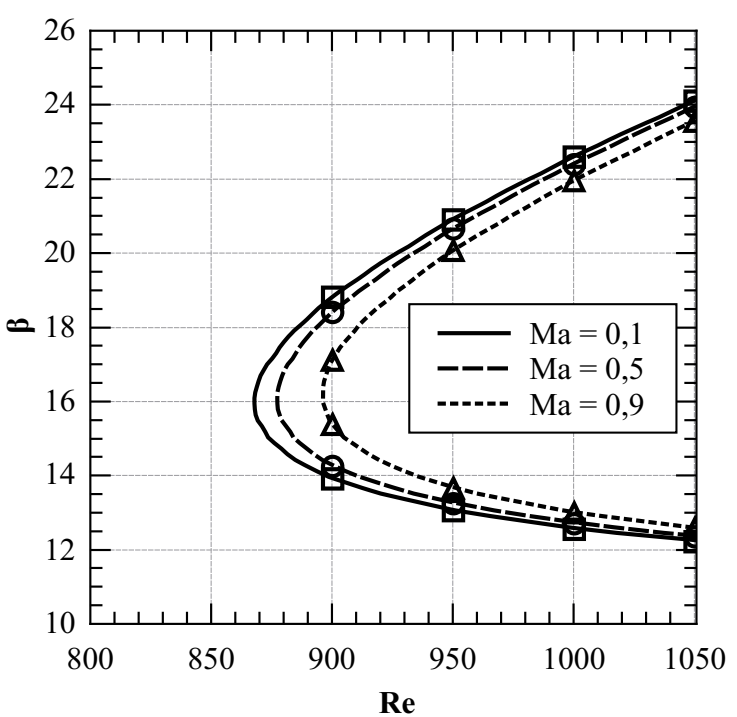

(b) Modo T1.

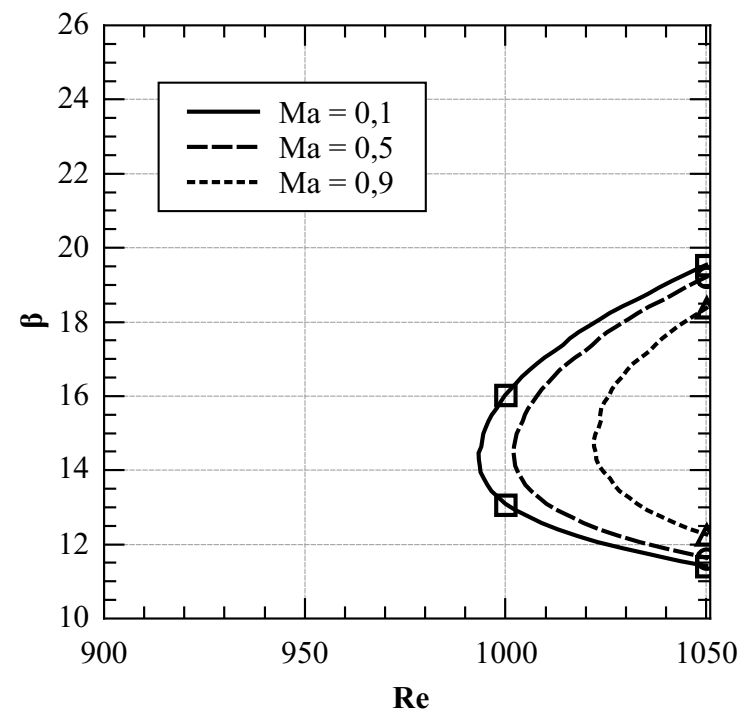

(d) Modo T3.

Figura 4.19: Curvas neutras para os quatro modos dominantes da cavidade compressível. 


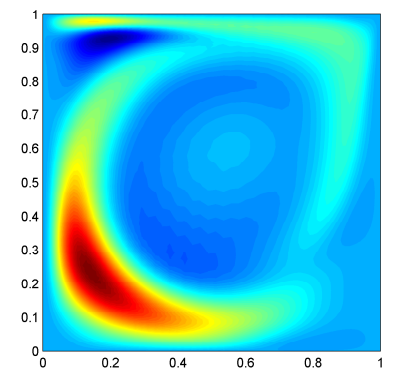

(a) $\hat{u}_{r}$

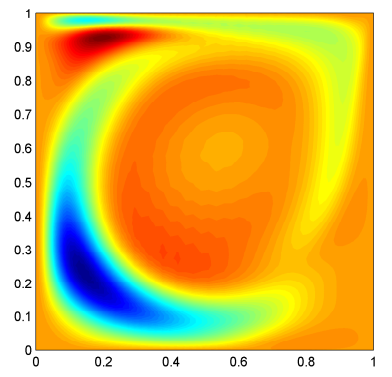

(e) $\hat{u}_{i}$

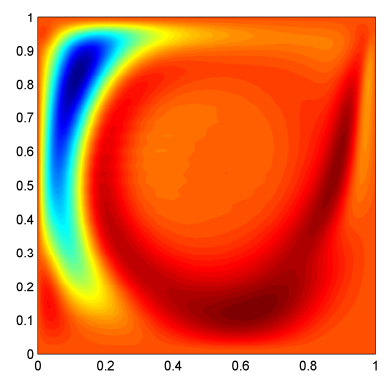

(b) $\hat{v}_{r}$

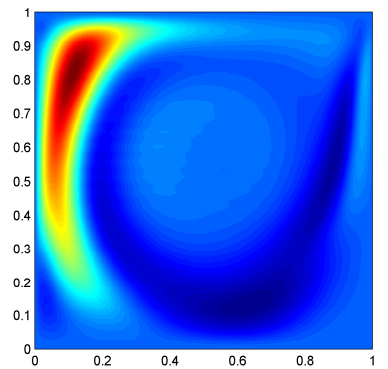

(f) $\hat{v}_{i}$

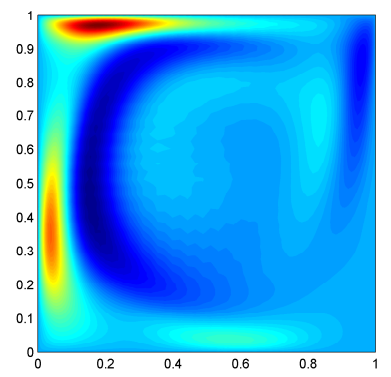

(c) $\hat{w}_{r}$

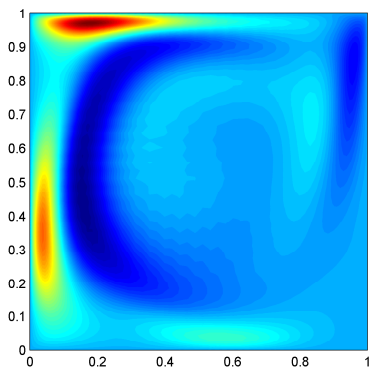

(g) $\hat{w}_{i}$

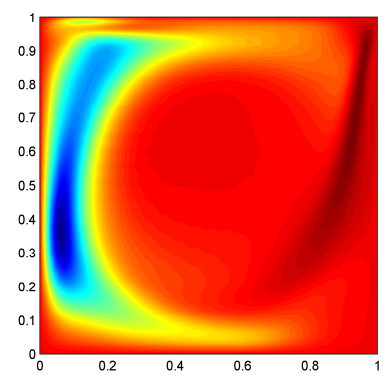

(d) $\hat{T}_{r}$

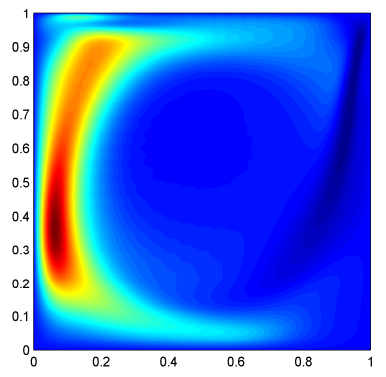

(h) $\hat{T}_{i}$

Figura 4.20: Modo S1 para $R e=900, M_{\infty}=0,5, \beta=15$.

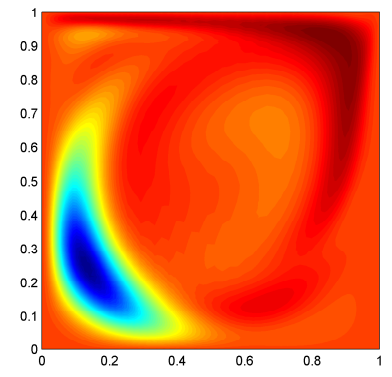

(a) $\hat{u}_{r}$

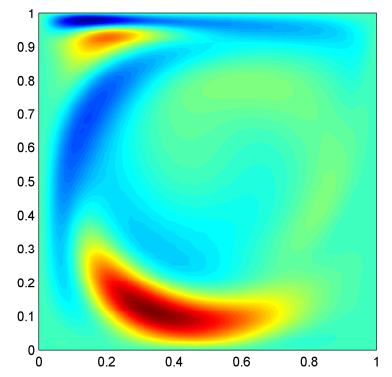

(e) $\hat{u}_{i}$

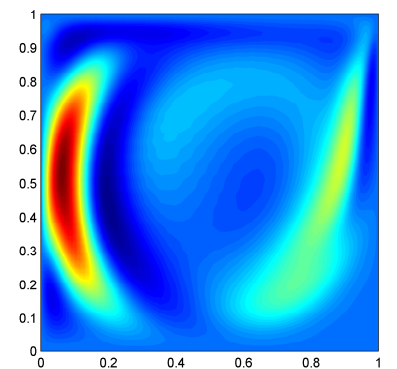

(b) $\hat{v}_{r}$

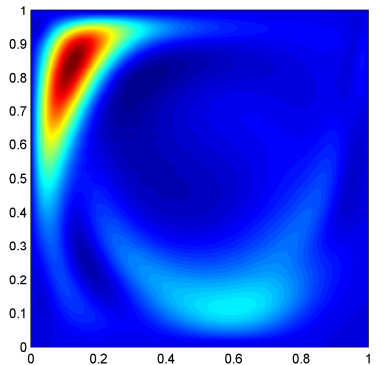

(f) $\hat{v}_{i}$

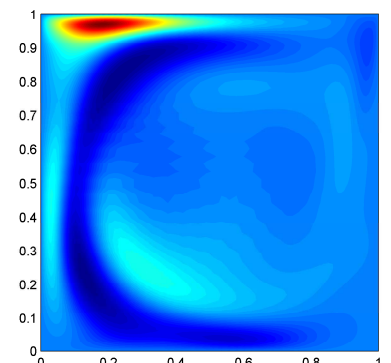

(c) $\hat{w}_{r}$

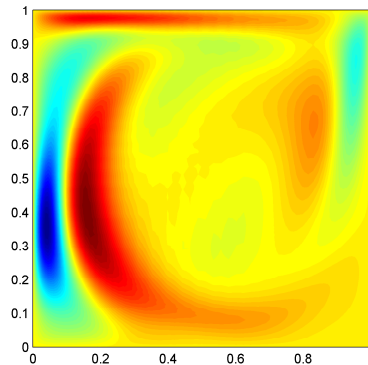

(g) $\hat{w}_{i}$

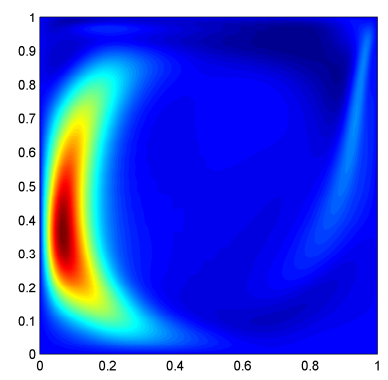

(d) $\hat{T}_{r}$

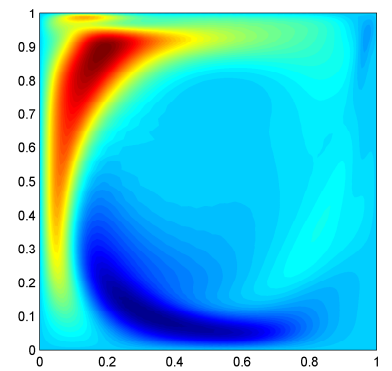

(h) $\hat{T}_{i}$

Figura 4.21: Modo T1 para $R e=900, M_{\infty}=0,5, \beta=15$. 


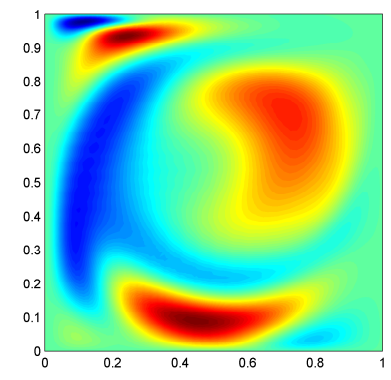

(a) $\hat{u}_{r}$

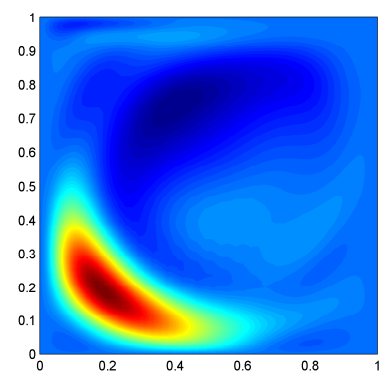

(e) $\hat{u}_{i}$

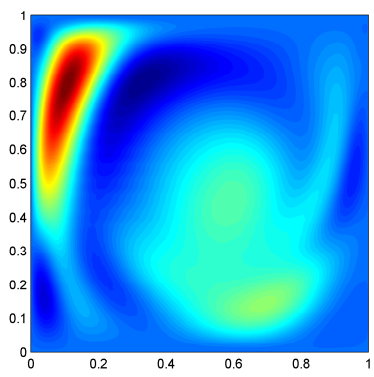

(b) $\hat{v}_{r}$

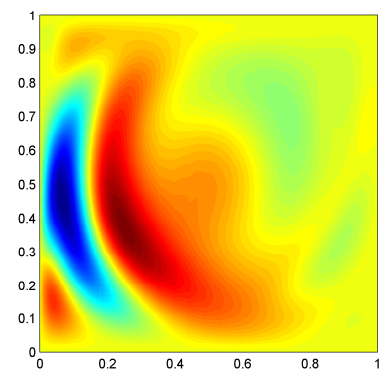

(f) $\hat{v}_{i}$

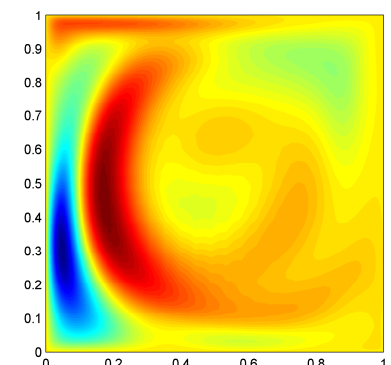

(c) $\hat{w}_{r}$

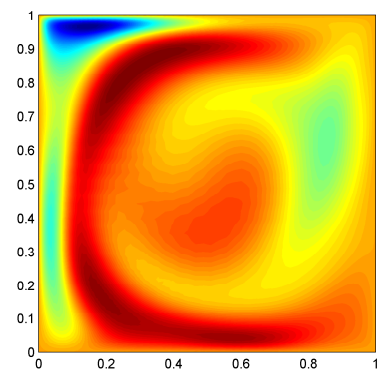

(g) $\hat{w}_{i}$

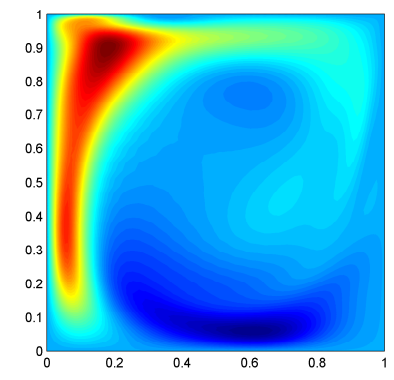

(d) $\hat{T}_{r}$

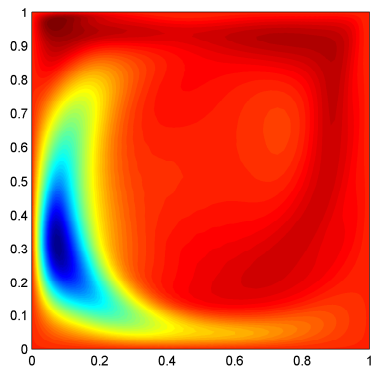

(h) $\hat{T}_{i}$

Figura 4.22: Modo T2 para $R e=900, M_{\infty}=0,5, \beta=7$.

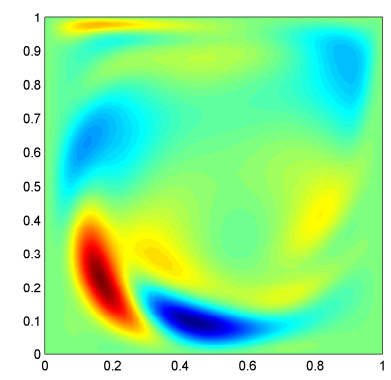

(a) $\hat{u}_{r}$

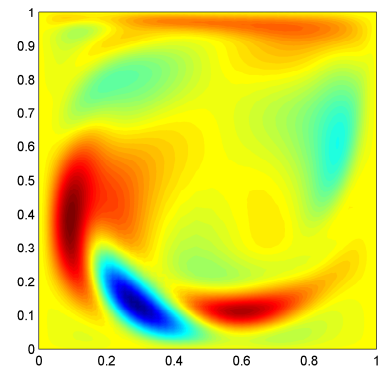

(e) $\hat{u}_{i}$

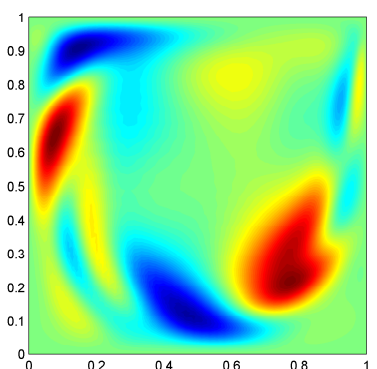

(b) $\hat{v}_{r}$

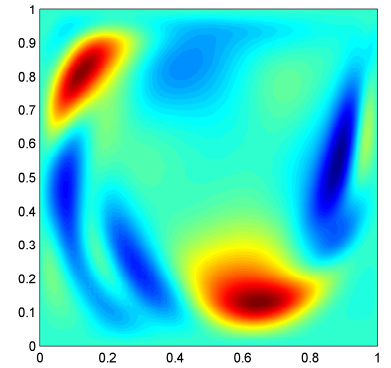

(f) $\hat{v}_{i}$

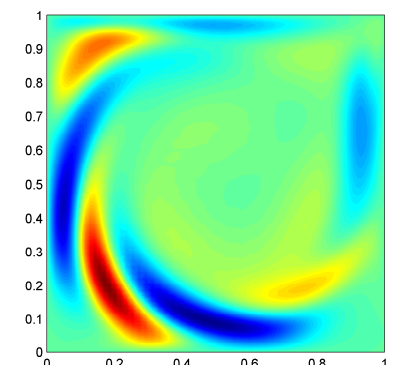

(c) $\hat{w}_{r}$

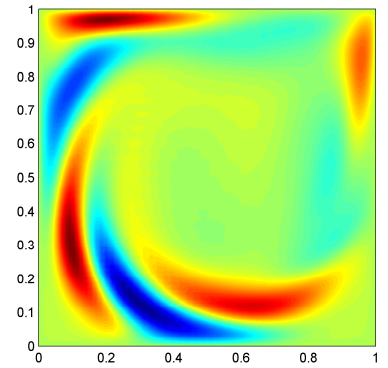

(g) $\hat{w}_{i}$

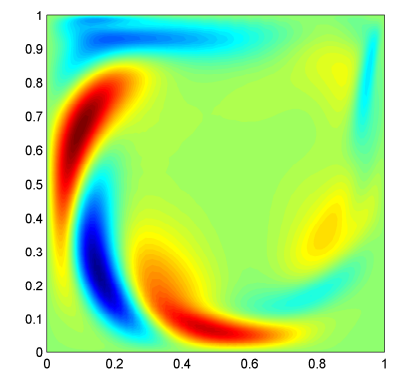

(d) $\hat{T}_{r}$

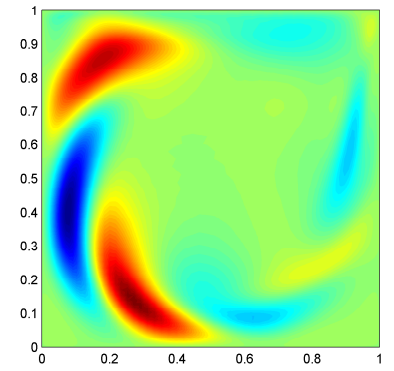

(h) $\hat{T}_{i}$

Figura 4.23: Modo T3 para $R e=900, M_{\infty}=0,5, \beta=15$. 


\section{Modos Acústicos de Duto}

Existem alguns modos inerentes ao escoamento compressível. Ou seja, ao se analisar o escoamento incompressível equivalente, esses modos não aparecem. Alguns desses modos são representados na figura 4.24 por círculos preenchidos. Os demais são modos encontrados tanto no escoamento compressível quanto no incompressível. A malha de $70 x 70$ pontos também foi adequada para discretizar estes modos compressíveis, como é visto no teste de convergência de malha apresentado na figura 4.25 .

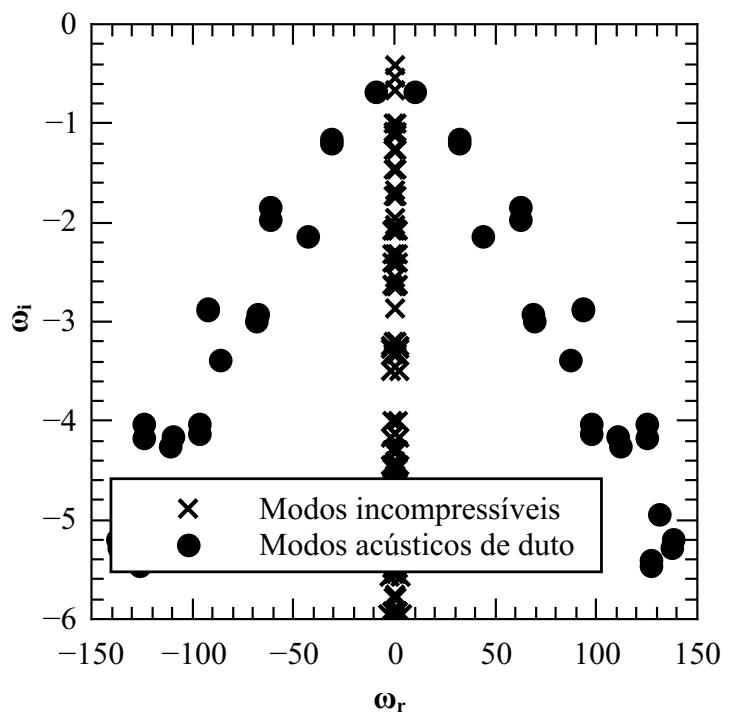

Figura 4.24: Modos compressíveis para $R e=100, M_{\infty}=0,1, \beta=1$.

Os modos compressíveis vistos na figura 4.24 são, na verdade, os modos de propagação acústica em um duto. Ou seja, são ondas acústicas propagando na direção $z$. Os números de onda dos modos de propagação em um duto quadrado de lado $L$, com escoamento base em repouso, é dado teoricamente por:

$$
\beta_{m, n}=\sqrt{\frac{\omega^{2}}{c^{2}}-\frac{\pi^{2}}{L^{2}}\left(m^{2}+n^{2}\right)},
$$

onde $0 \leq m, n \leq \infty$.

Para um determinado número de onda, as frequências dos modos de propagação em duto 


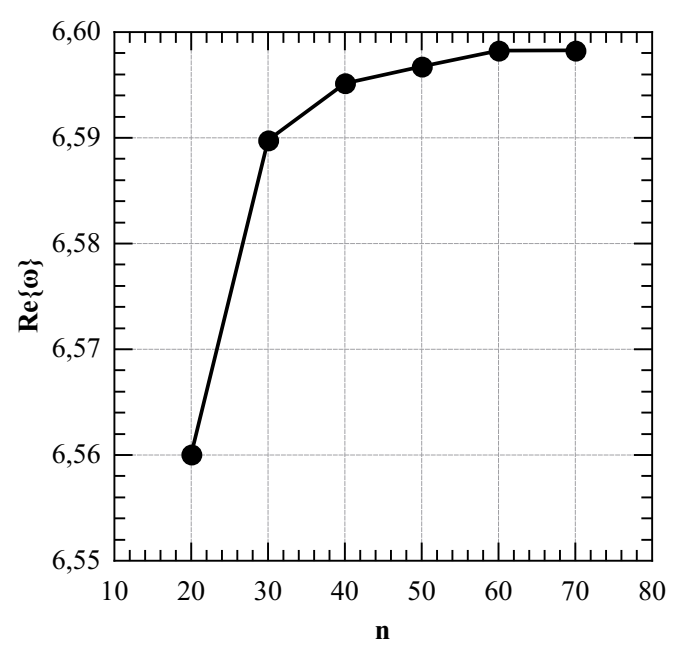

(a) $\omega_{r}$.

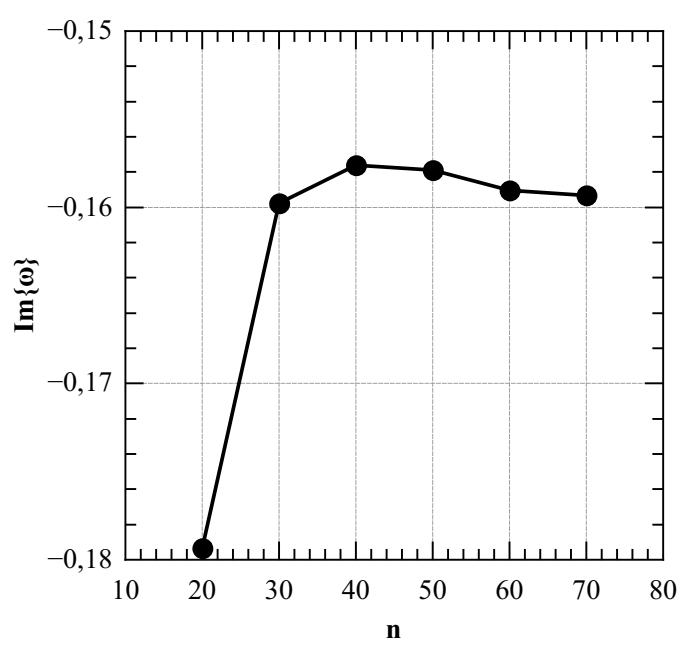

(b) $\omega_{i}$.

Figura 4.25: Convergência de malha para o modo compressível dominante. $R e=900, M_{\infty}=$ $0,5, \beta=0$.

são dadas por:

$$
\omega=c \sqrt{\beta^{2}+\frac{\pi^{2}}{L^{2}}\left(m^{2}+n^{2}\right)} .
$$

Uma boa correlação é obtida ao se comparar a parte real dos autovalores compressívies mostrados na figura 4.24 com o resultado obtido em (4.10) para $\beta=1$ e $c=\frac{1}{M_{\infty}}=10$, como é mostrado na figura 4.26 .

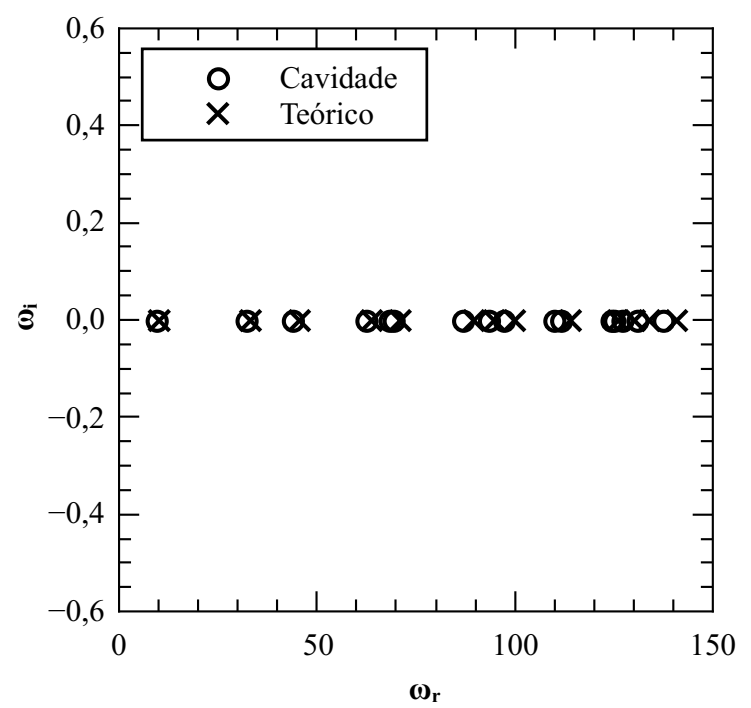

Figura 4.26: Comparação dos modos compressíveis da cavidade a $\operatorname{Re}=100, M_{\infty}=0,1, \beta=1$ com os modos de propagação acústica em duto.

A autofunção de um destes modos acústicos é mostrada na figura 4.27. 
Ao contrário do que ocorre com os modos incompressívies, a compressibilidade tem efeito desestabilizante nos modos acústicos, como é mostrado na figura 4.28. Ainda assim, estes modos são sempre estáveis para os números de Reynolds, Mach e números de onda verificados e são menos estáveis para $\beta$ próximo de zero, como é mostrado na figura 4.29. 


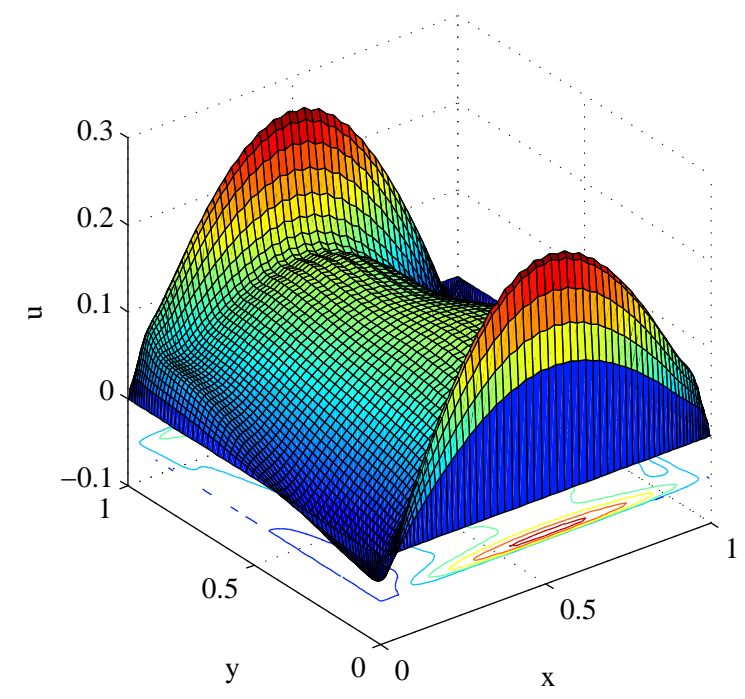

(a) $\hat{u}_{r}$

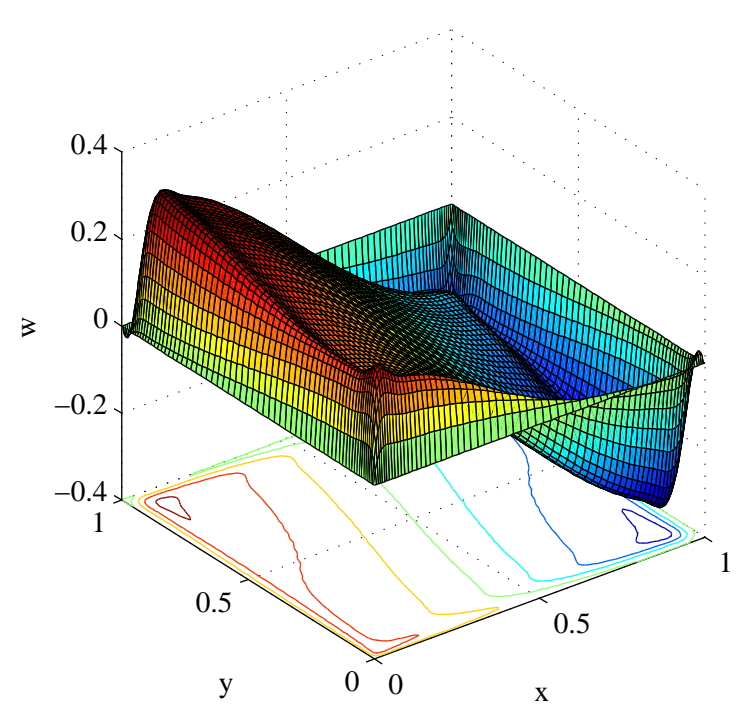

(c) $\hat{w}_{r}$

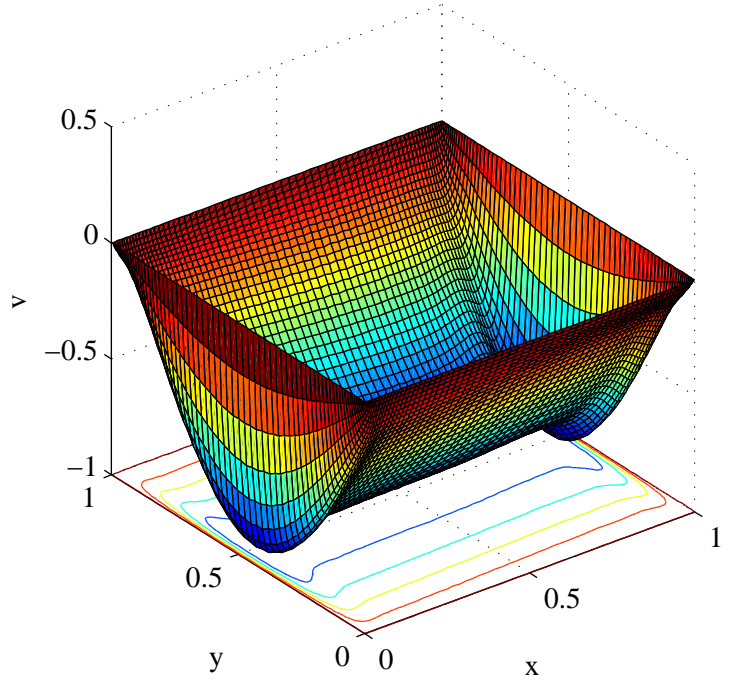

(b) $\hat{v}_{r}$

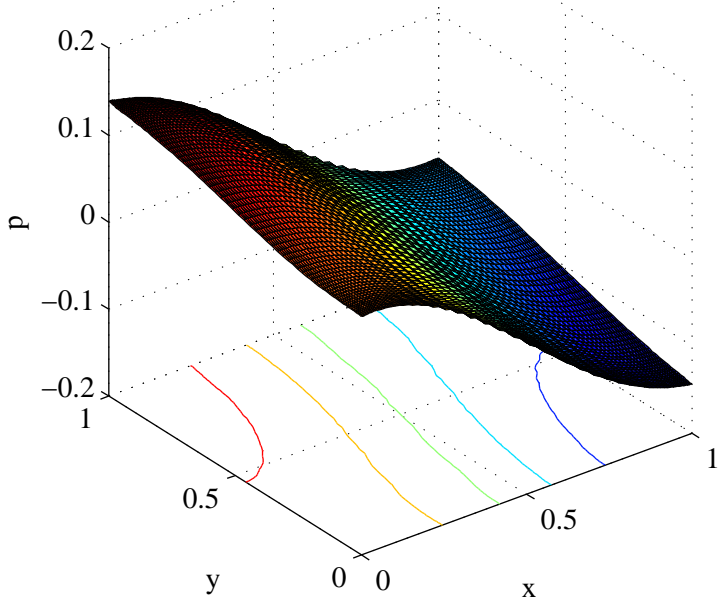

(d) $\hat{p}_{r}$

Figura 4.27: Parte real das autofunções do modo de propagação acústica em duto. $\omega=31,907-$ 1,154i. $R e=100, M_{\infty}=0,1, \beta=0$. 


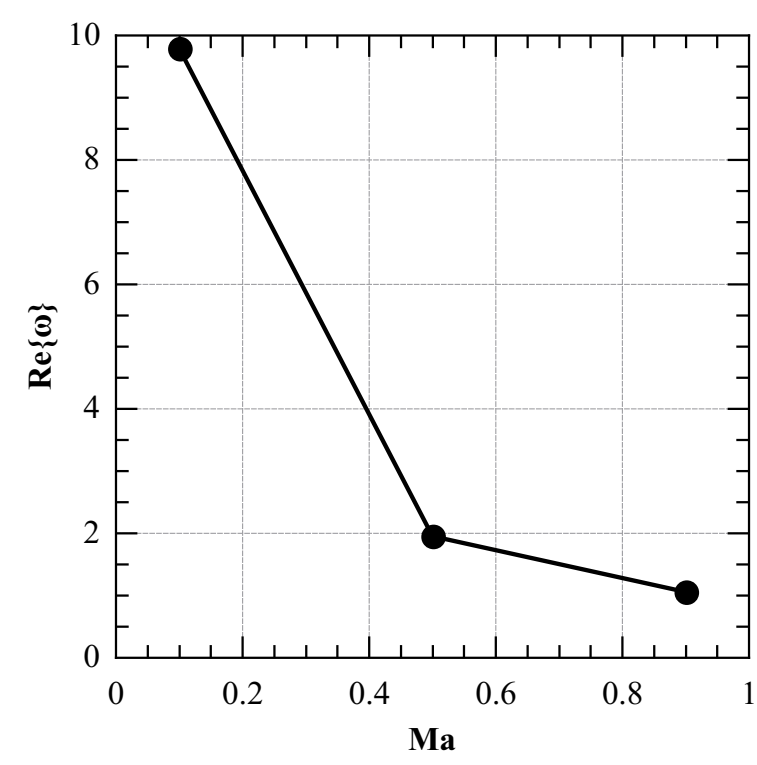

(a) $u$

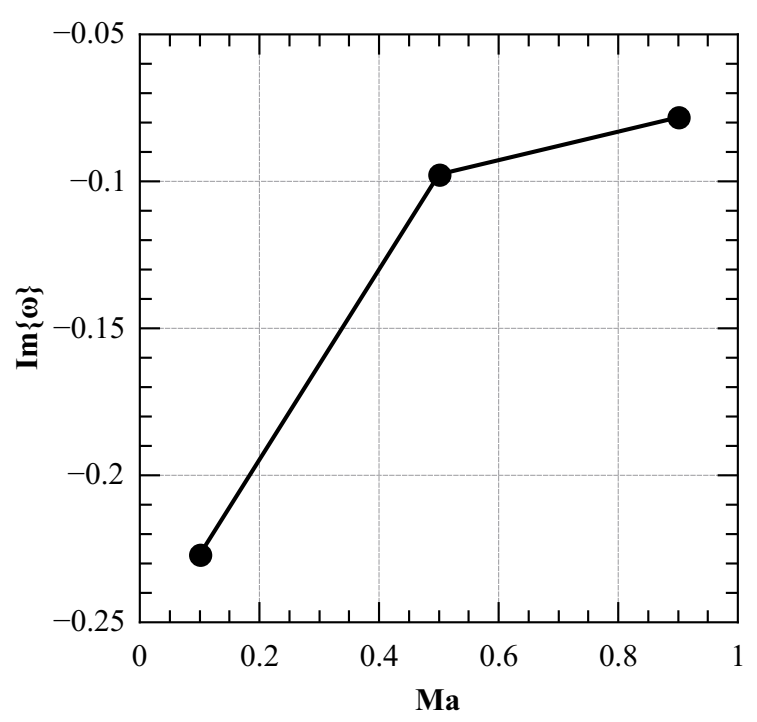

(b) $v$

Figura 4.28: Influência da compressibilidade nos modos de propagação acústica em duto. $R e=$ $900, \beta=1$.

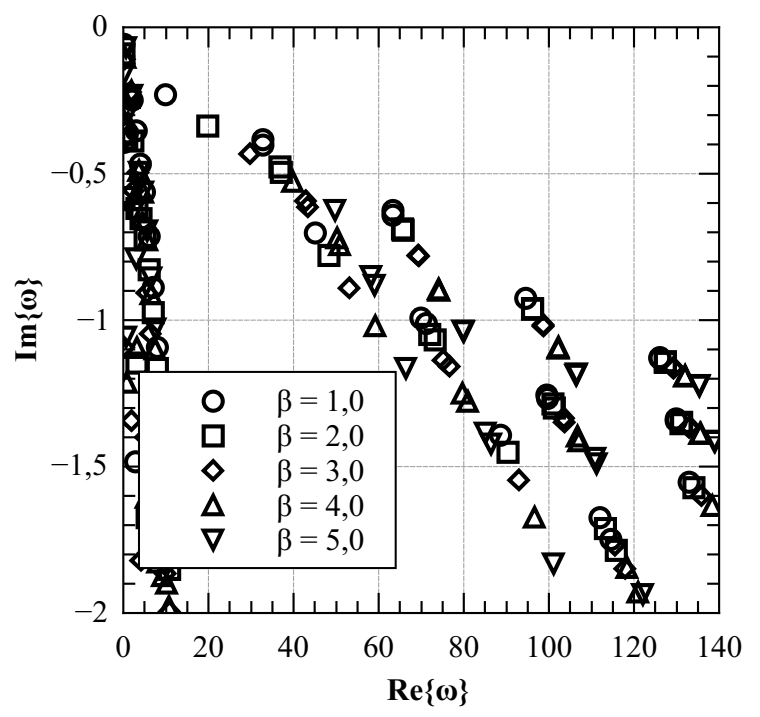

Figura 4.29: Modos compressivies para $R e=900, M_{\infty}=0,5$. Variação em $\beta$. 


\section{Modos Acústicos de Cavidade}

Dentre os modos compressíveis existe um ramo (figura 4.30) cujos modos estão relacionados à geração de som no canto superior direito da cavidade. Isto foi inferido pela forma das autofunções destes modos, que têm a forma de uma onda sonora se propagando a partir da quina, com a amplitude bem maior na região onde é localizada a fonte sonora, como é visto na figura 4.31. De fato, o comprimento de onda nas regiões mais distantes da fonte sonora é muito próximo ao comprimento de uma onda acústica propagando-se no campo distante. Isto é verificado pela relação de dispersão:

$$
\omega_{r}=\frac{2 \pi c}{\lambda_{p}}
$$

onde $\lambda_{p}$ é o comprimento de onda.

Para o modo acústico de cavidade apresentado na figura 4.30 temos $\omega_{r}=65,756$ e $c=$ $1 / M_{\infty}=2$. O comprimento de onda associado é, portanto, $\lambda_{p}=0,1911$. Este comprimento de onda é observado na autofunção para as regiões distantes da fonte (figura 4.33).

Os modos acústicos de cavidade são estáveis para os números de Reynolds, Mach e números de onda verificados. Observa-se que o aumento do número de onda torna estes modos mais estáveis, assim como a redução do número de Mach, como pode ser observado na figura 4.32.

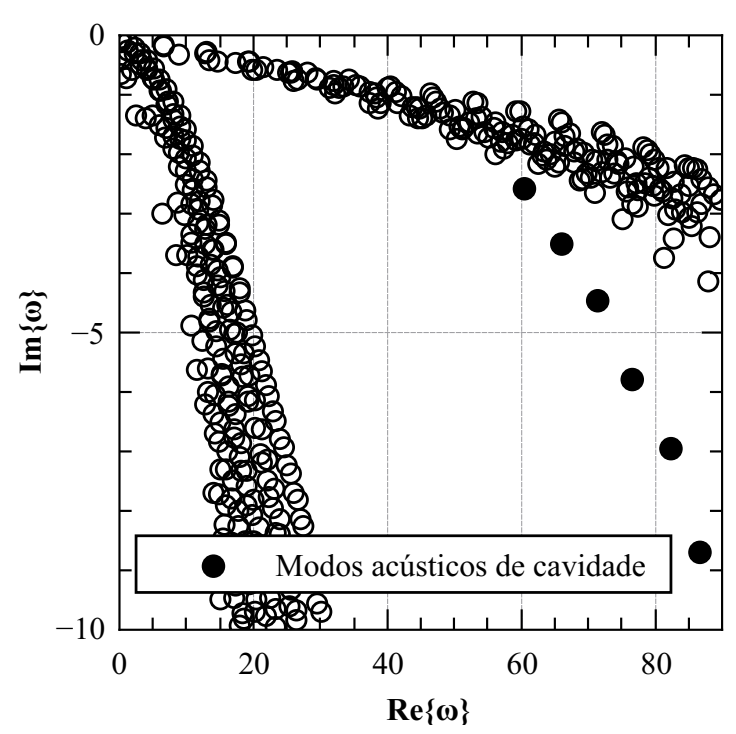

Figura 4.30: Modos acústicos de cavidade para $R e=900, M_{\infty}=0,5, \beta=0$. 


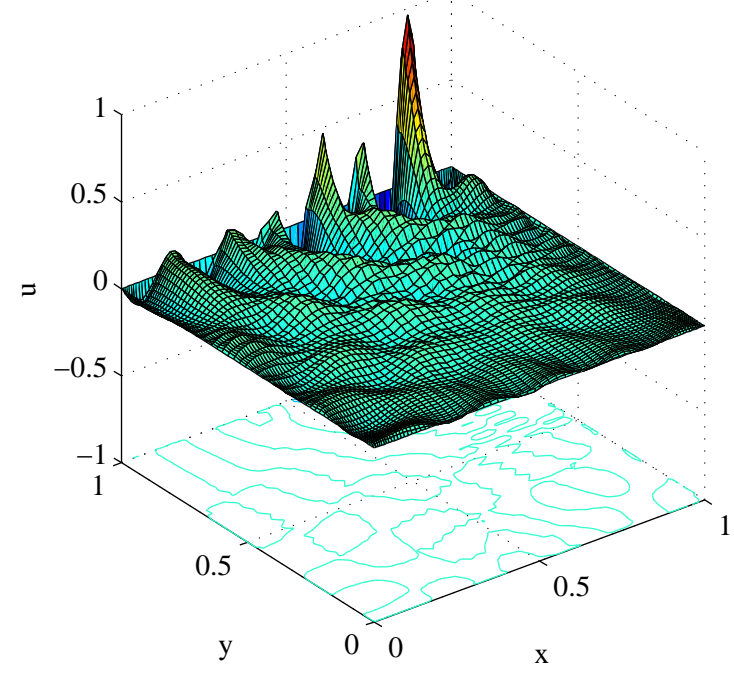

(a) $\hat{u}_{r}$

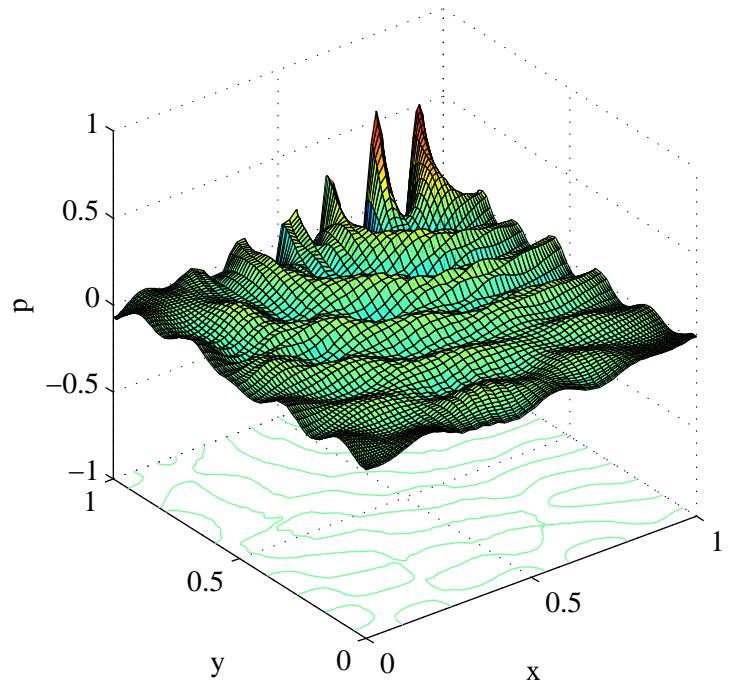

(c) $\hat{p}_{r}$

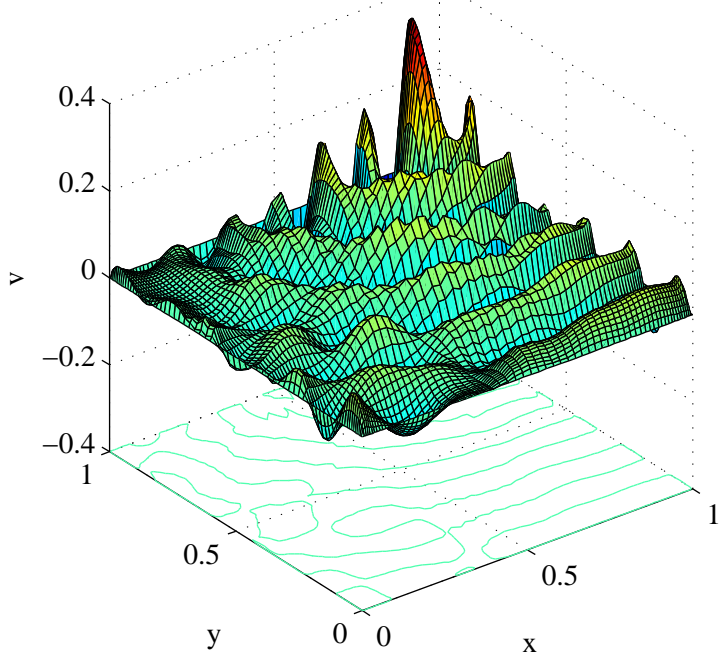

(b) $\hat{v}_{r}$

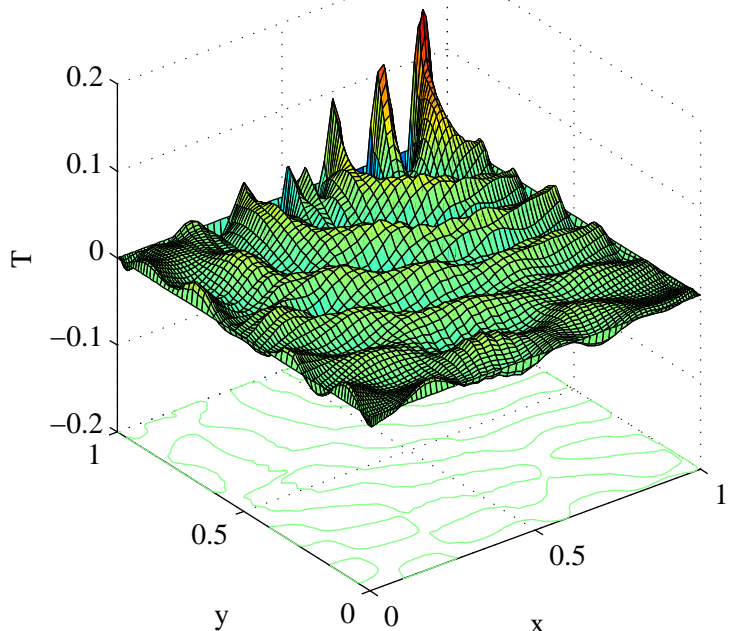

(d) $\hat{T}_{r}$

Figura 4.31: Parte real das autofunções do modo acústico de cavidae $\omega=65.756-3.4987 i$. $R e=900, M_{\infty}=0,5, \beta=0$. 


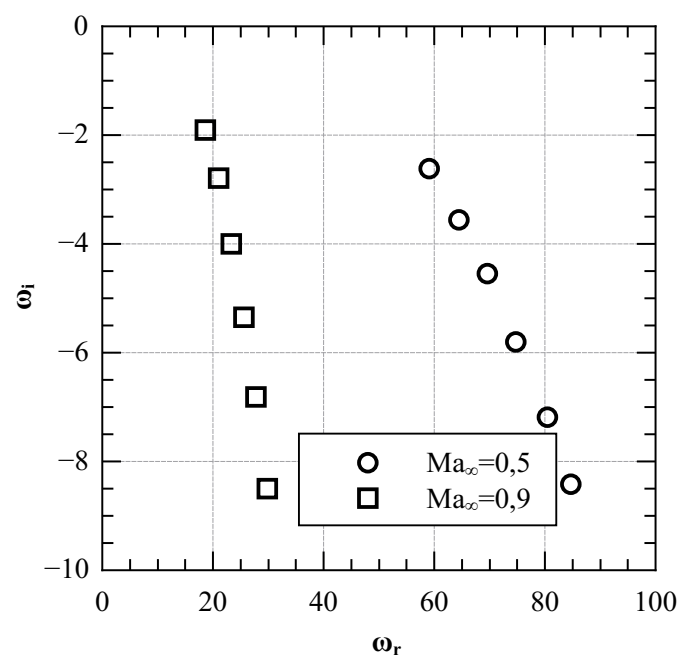

(a) $\beta=1$

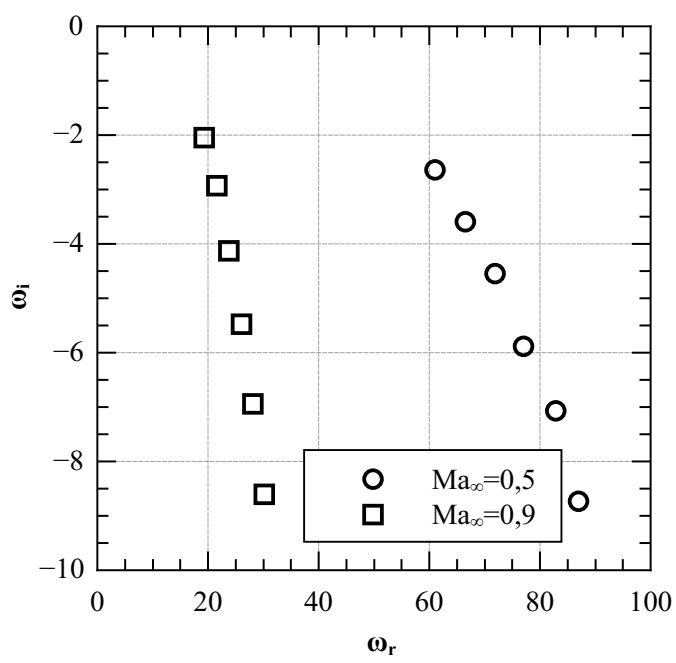

(b) $\beta=5$

Figura 4.32: Variação dos autovalores dos modos acústicos de cavidade com Mach e $\beta$ para $R e=900$.

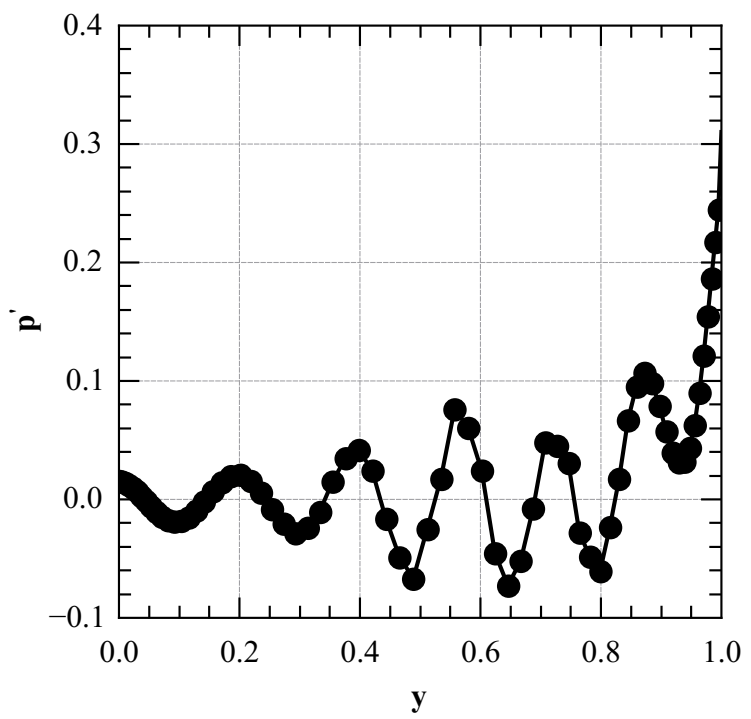

Figura 4.33: $p^{\prime}$ ao longo de $x=0,947 . R e=900, M_{\infty}=0,5, \beta=0$. 


\section{Pseudo Espectro}

O operador linear para a cavidade apresenta grande não normalidade, estando sujeito a fenômenos como crescimento transiente para certas condições iniciais. Este tópico não foi explorado nesta dissertação, restringindo-se a análise do pseudo espectro. O número de Mach não causa grandes alterações no pseudo espectro, como pode ser visto nas figuras 4.34 a 4.36. Este é um indício de que o crescimento transiente não deve sofrer variações sensíveis com a compressibilidade.

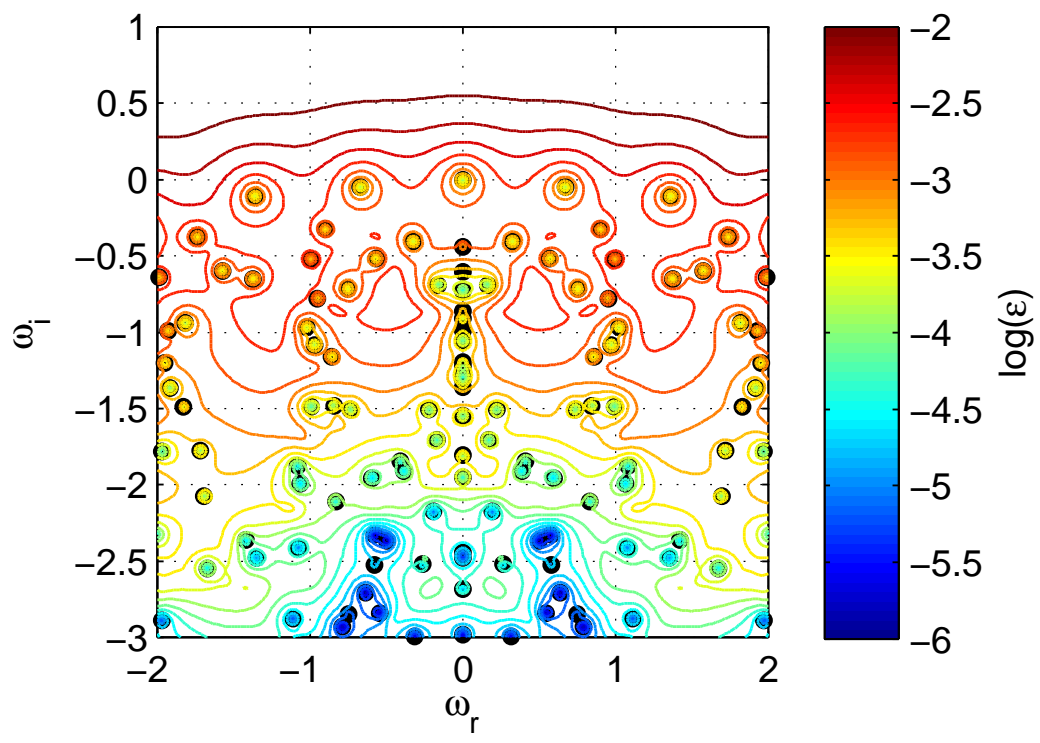

Figura 4.34: Pseudo espectro para $M_{\infty}=0,1 . \operatorname{Re}=800, \beta=15$. 


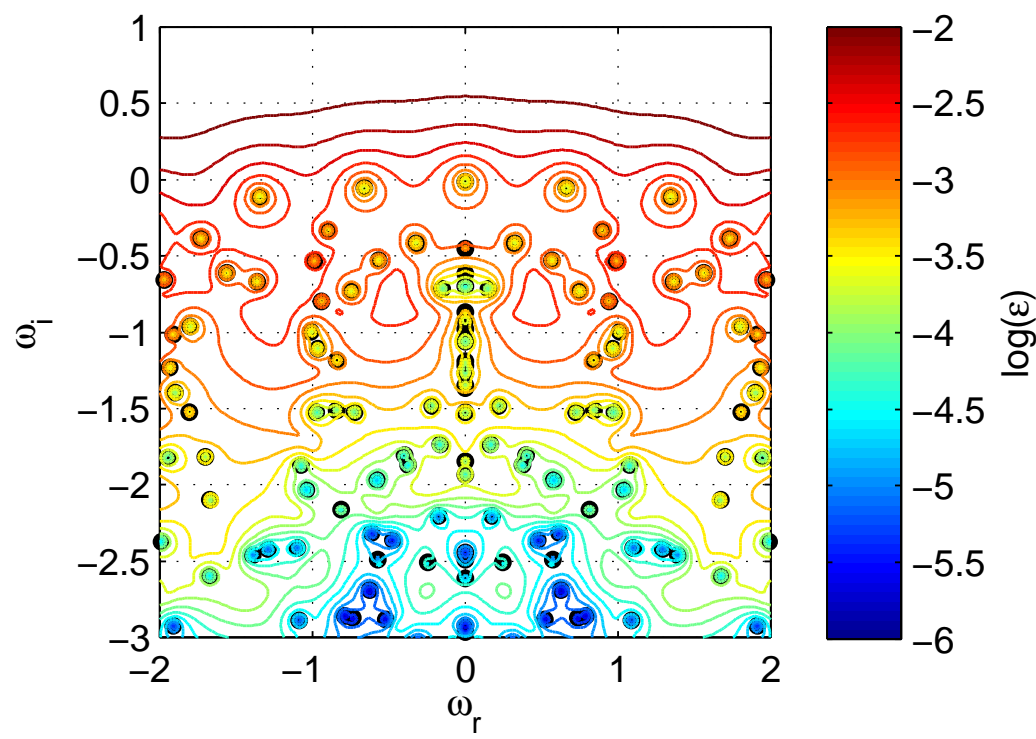

Figura 4.35: Pseudo espectro para $M_{\infty}=0,5 . R e=800, \beta=15$.

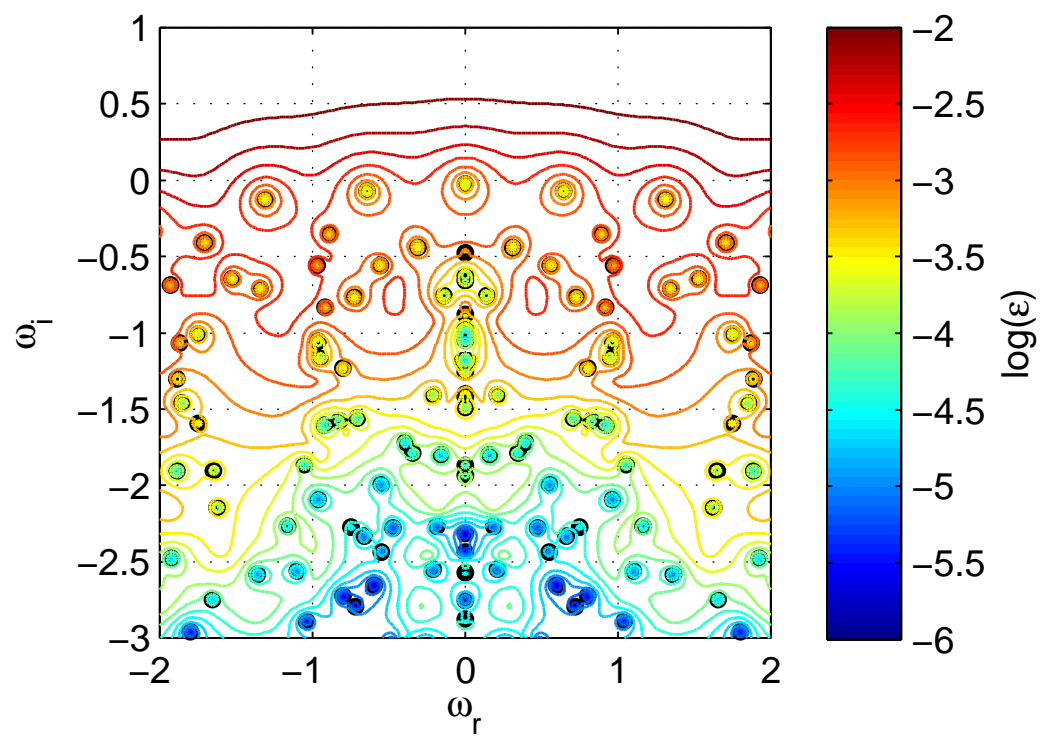

Figura 4.36: Pseudo espectro para $M_{\infty}=0,9 . \operatorname{Re}=800, \beta=15$. 


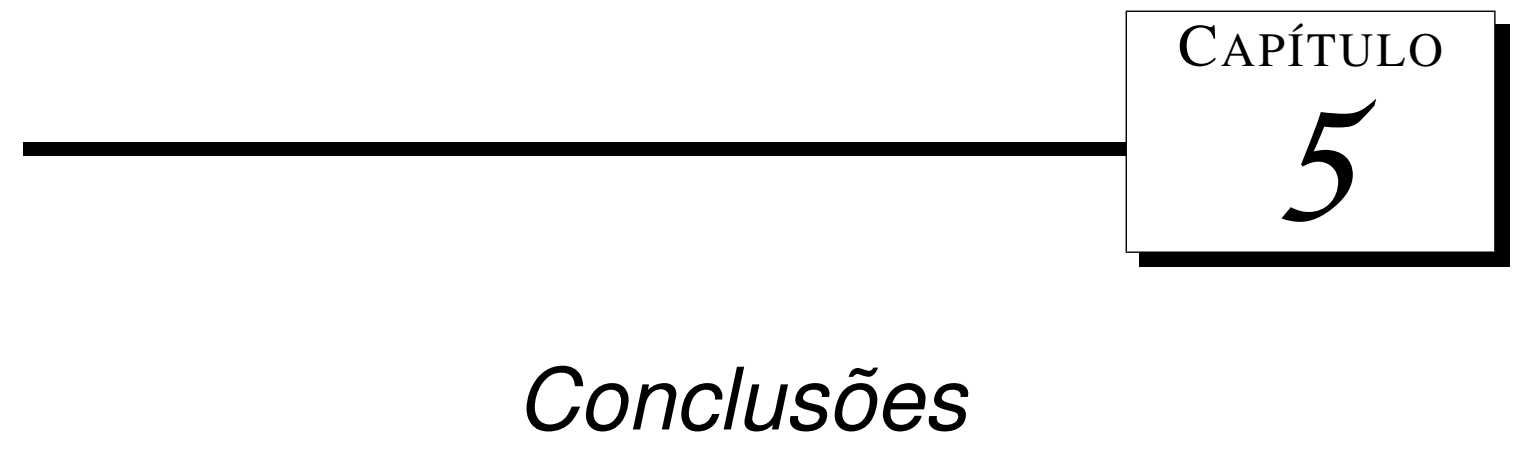

Esta dissertação abordou a instabilidade hidrodinâmica linear do escoamento compressível no interior de uma cavidade com tampa deslizante.

O escoamento base foi obtido através de um código DNS desenvolvido para este trabalho. Neste código foram empregadas diferenças finitas compactas com alta resolução espectral para a discretização espacial em uma malha retilínea não uniforme. Um filtro compacto foi utilizado para evitar o surgimento de ondas espúrias de alta frequência e a integração temporal da solução foi feita pelo método de Runge Kutta de quarta ordem. O código permite o cálculo de escoamentos tridimensionais e tem capacidade de processamento processamento paralelo através da divisão do domínio nas três direções, ou em duas, no caso bidimensional. A divisão do domínio foi realizada em três etapas, onde em cada etapa uma direção foi preservada de forma a possibilitar o uso das diferenças finitas compactas sem perda de precisão.

A instabilidade hidrodinâmica foi analisada pelo método dito biglobal, onde as perturbações tridimensionais são decompostas em modos não homogêneos em duas direções e periódicos na terceira. Um problema de autovalor generalizado bidimensional deve ser resolvido para o cálculo do espectro, demandando uma grande quantidade de memória. Para tornar a resolução do problema de autovalor viável, o código foi desenvolvido com algoritmos para matrizes esparsas que, junto com a discretização por diferenças finitas explícitas, reduz sensivelmente a memória necessária. Além disso, o algoritmo de Arnoldi foi usado para reduzir o problema original para 
um problema equivalente contendo os autovalores mais importantes.

Observou-se que a compressibilidade tem um efeito estabilizante nos modos já conhecidos do problema incompressível associado, ou seja, aumentando o Reynolds crítico. Aqui foram verificados apenas casos subsônicos. A compressibilidade também não altera a ordem em que estes modos tornam-se instáveis e tem uma influência mínima nos respectivos números de onda críticos. Dentre os 4 modos dominantes, comparando-se os escoamentos a Mach 0,1 e 0,9, aquele que teve maior aumento do Reynolds crítico foi o modo estacionário (S1), da ordem de 3,5\%. A maior variação no número de onda crítico foi observada no modo T3, sendo da ordem de $2,3 \%$.

Existem modos intrínsecos ao escoamento compressível na cavidade. Estes modos compressíveis tornam-se menos estáveis com o aumento do número de Mach, no entanto são sempre estáveis para os números de Reynolds avaliados. Também ficam menos estáveis para números de onda pequenos, se aproximando do caso bidimensional.

Um conjunto dos modos compressíveis está relacionado à propagação de som no interior de um duto, ou seja, na direção $z$ da cavidade. Existe também ramo distinto, contendo modos relacionados à geração e propagação de som a partir do canto superior direito da cavidade, onde o escoamento acelerado pela tampa encontra a parede lateral. Estes modos também permaneceram sempre estáveis para as condições estudadas, embora fiquem menos estáveis com o aumento do número de Mach. Não foram encontrados registros sobre estes modos na literatura.

Através da análise do pseudo espectro, observou-se que a cavidade com tampa deslizante está sujeita ao fenômeno do crescimento transiente, embora a compressibilidade não altere sensivelmente esta característica.

Como uma sequência deste trabalho, pode-se analisar a instabilidade do escoamento no interior da cavidade para regimes transônicos e supersônicos. Outros tópicos a serem explorados são a caracterização do crescimento transiente e a instabilidade hidrodinâmica no escoamento compressível de uma cavidade aberta. 


\title{
Referências Bibliográficas
}

\author{
AIDUN, C. K.; TRIANTAFILLOPOULOS, N. G.; BENSON, J. D. Global stability of a \\ lid-driven cavity with throughflow: Flow visualization studies. Physics of Fluids A: Fluid \\ Dynamics, v. 3, n. 9, p. 2081, 1991. ISSN 08998213.
}

ARNAL, D. Description and prediction of transition in two-dimensional, incompressible flow. In: In AGARD Spec. Course on Stability and Transition of Laminar Flow 71 p (SEE N84-33757 23-34). [S.1.: s.n.], 1984. v. 1.

COLONIUS, T.; LELE, S. K. Computational aeroacoustics: progress on nonlinear problems of sound generation. Progress in Aerospace Sciences, v. 40, n. 6, p. 345-416, ago. 2004. ISSN 03760421.

CRIMINALE, W.; JACKSON, T.; JOSLIN, R. Theory and computation of hydrodynamic stability. [S.1.]: Cambridge University Press, 2003.

DING, Y.; KAWAHARA, M. Three-dimensional linear stability analysis of incompressible viscous flows using the finite element method. ... journal for numerical methods in fluids, v. 479, n. April 1997, p. 451-479, 1999.

DRAZIN, P. G.; REID, W. H. Introduction to hydrodynamic stability. Applied Mechanics Reviews, v. 56, p. B43, 2003.

GAITONDE, D.; VISBAL, M. High-order schemes for Navier-Stokes equations: algorithm and implementation into FDL3DI. [S.1.], 1998.

GASTER, M. A note on the relation between temporally-increasing and spatially-increasing disturbances in hydrodynamic stability. J. Fluid Mech, v. 14, n. 14, p. 222-224, 1962.

GENNARO, E. M. et al. Sparse Techniques in Global Flow Instability with Application to Compressible Leading-Edge Flow. AIAA Journal, v. 51, n. 9, p. 1-9, jul. 2013. ISSN 0001-1452.

GHIA, U.; GHIA, K.; SHIN, C. High-Re solutions for incompressible flow using the Navier-Stokes equations and a multigrid method. Journal of computational physics, v. 411, n. 3, p. 387-411, 1982.

GOODRICH, J. W.; GUSTAFSON, K.; HALASI, K. Hopf bifurcation in the driven cavity. Journal of Computational Physics, v. 90, n. 1, p. 219-261, set. 1990. ISSN 00219991.

HEEG, R.; GEURTS, B. Spatial instabilities of the incompressible attachment-line flow using sparse matrix Jacobi-Davidson techniques. Applied Scientific Research, Springer, v. 59, n. 4, p. 315-329, 1997. ISSN 0003-6994. 
HERMANNS, M. Stable high-order finite-difference methods based on non-uniform grid point distributions. Journal for Numerical Methods, v. 56, n. May 2007, p. 233-255, 2008.

HUERRE, P.; MONKEWITZ, P. Local and global instabilities in spatially developing flows. Annual Review of Fluid Mechanics, Annual Reviews 4139 El Camino Way, PO Box 10139, Palo Alto, CA 94303-0139, USA, v. 22, n. 1, p. 473-537, jan. 1990. ISSN 0066-4189.

JACOBS, G. B.; KOPRIVA, D. a.; MASHAYEK, F. A Conservative Isothermal Wall Boundary Condition for the Compressible Navier-Stokes Equations. Journal of Scientific Computing, v. 30, n. 2, p. 177-192, dez. 2005. ISSN 0885-7474.

LELE, S. Compact finite difference schemes with spectral-like resolution. Journal of Computational Physics, Elsevier, v. 103, n. 1, p. 16-42, 1992.

LI, N.; LAIZET, S. 2DECOMP\&FFT - A Highly Scalable 2D Decomposition Library and FFT Interface. In: Cray User Group 2010 conference, Edinburgh, 2010. [S.1.]: Cray User Group, 2010. p. 1-13.

MENDONCA, M. T.; MEDEIROS, M. A. F. Instabilidade hidrodinâmica e transição para turbulênica com aplicações em engenharia e meteorologia. [S.1.]: ., 2009.

MORGAN, P.; VISBAL, M.; RIZZETTA, D. A Parallel Overset Grid High-Order Flow Solver for Large Eddy Simulation. Journal of Scientific Computing, v. 29, n. 2, p. 165-200, out. 2006. ISSN 0885-7474.

ORR, W. M. The stability or instability of the steady motions of a perfect liquid and of a viscous liquid. part i: A perfect liquid. v. 27, p. 9-68, 1907.

ORR, W. M. The stability or instability of the steady motions of a perfect liquid and of a viscous liquid. part ii: A viscous liquid. v. 27, p. 69-138, 1907.

REYNOLDS, O. An experimental investigation of the circumstances which determine whether the motion of water shall be direct or sinuous, and of the law of resistance in parallel channels. Proceedings of the Royal Society of London, The Royal Society, v. 35, n. 224-226, p. 84-99, 1883.

SAAD, Y. Variations on Arnoldi's method for computing eigenelements of large unsymmetric matrices. Linear Algebra and its Applications, v. 34, n. 1, p. 269-295, dez. 1980. ISSN 00243795 .

SCHLICHTING, H. Zur Entstehung der Turbulenz bei der Plattenströmung. Nachrichten von der Gesellschaft der Wissenschaften zu Göttingen, p. 181-208, 1933.

SCHMID, P. J. Nonmodal Stability Theory. Annual Review of Fluid Mechanics, v. 39, n. 1, p. 129-162, jan. 2007. ISSN 0066-4189.

SCHUBAUER, G. B.; SKRAMSTAD, H. K. Laminar-boundary-layer oscillations and transition on a flat plate. NATIONAL AERONAUTICS AND SPACE ADMINISTRATION WASHINGTON DC, 1948.

SHAH, P. et al. Subsonic compressible flow in two-sided lid-driven cavity. Part I: Equal walls temperatures. International Journal of Heat and Mass Transfer, v. 50, n. 21-22, p. 4206-4218, out. 2007. ISSN 00179310. 
SHANKAR, P.; DESHPANDE, M. Fluid mechanics in the driven cavity. Annual Review of Fluid Mechanics, Annual Reviews 4139 El Camino Way, PO Box 10139, Palo Alto, CA 94303-0139, USA, v. 32, n. 1, p. 93-136, 2000.

SHEN, J. Hopf bifurcation of the unsteady regularized driven cavity flow. Journal of Computational Physics, v. 245, n. 199 1, p. 228-245, 1991.

SOMMERFELD, A. Ein beitrag zur hydrodynamischen erklärung der turbulenten flüssigkeitsbewegungen. Atti del, v. 4, p. 116-124, 1908.

THEOFILIS, V. Advances in global linear instability analysis of nonparallel and threedimensional flows. Progress in Aerospace Sciences, v. 39, n. 4, p. 249-315, maio 2003. ISSN 03760421 .

THEOFILIS, V.; DUCK, P.; OWEN, J. Viscous linear stability analysis of rectangular duct and cavity flows. Journal of Fluid Mechanics, Cambridge Univ Press, v. 505, p. 249-286, 2004.

TOLLMIEN, W.; SCHLICHTING, H.; GÖRTLER, H. Über die entstehung der turbulenz. Springer, p. 812-816, 1961.

TREFETHEN, L. et al. Hydrodynamic stability without eigenvalues. Science, v. 261, n. 5121, p. 578-584, 1993.

TREFETHEN, L. N. Pseudospectra of Linear Operators. SIAM Review, v. 39, n. 3, p. 383-406, jan. 1997. ISSN 0036-1445. 


\section{APÊNDICE A - Operador linear}

\section{incompressível}

$$
\mathscr{L}=\left[\begin{array}{cccc}
\mathscr{L}_{u, u} & \mathscr{L}_{u, v} & 0 & \mathscr{L}_{u, p} \\
\mathscr{L}_{v, u} & \mathscr{L}_{v, v} & 0 & \mathscr{L}_{v, p} \\
\mathscr{L}_{w, u} & \mathscr{L}_{w, v} & \mathscr{L}_{w, w} & \mathscr{L}_{w, p} \\
\mathscr{L}_{p, u} & \mathscr{L}_{p, v} & \mathscr{L}_{p, w} & 0
\end{array}\right]
$$

$$
\mathscr{R}=\left[\begin{array}{cccc}
\mathscr{R}_{u, u} & 0 & 0 & 0 \\
0 & \mathscr{R}_{v, v} & 0 & 0 \\
0 & 0 & \mathscr{R}_{w, w} & 0 \\
0 & 0 & 0 & 0
\end{array}\right]
$$

$$
\left\{\begin{array}{l}
\mathscr{L}_{u, u}=\frac{1}{R e}\left(\frac{\partial^{2}}{\partial x^{2}}+\frac{\partial^{2}}{\partial y^{2}}-\beta^{2}\right)-\bar{u} \frac{\partial}{\partial x}-\bar{v} \frac{\partial}{\partial y}-i \beta \bar{w}-\frac{\partial \bar{u}}{\partial x} \\
\mathscr{L}_{u, v}=-\frac{\partial \bar{u}}{\partial y} \\
\mathscr{L}_{u, p}=-\frac{\partial}{\partial x} \\
\mathscr{R}_{u, u}=-i
\end{array}\right.
$$


$\left\{\begin{array}{l}\mathscr{L}_{v, u}=-\frac{\partial \bar{v}}{\partial x} \\ \mathscr{L}_{v, v}=\frac{1}{R e}\left(\frac{\partial^{2}}{\partial x^{2}}+\frac{\partial^{2}}{\partial y^{2}}-\beta^{2}\right)-\bar{u} \frac{\partial}{\partial x}-\bar{v} \frac{\partial}{\partial y}-i \beta \bar{w}-\frac{\partial \bar{v}}{\partial y} \\ \mathscr{L}_{v, p}=-\frac{\partial}{\partial y} \\ \mathscr{R}_{v, v}=-i\end{array}\right.$

$\left\{\begin{array}{l}\mathscr{L}_{w, u}=-\frac{\partial \bar{w}}{\partial x} \\ \mathscr{L}_{w, v}=-\frac{\partial \bar{w}}{\partial y} \\ \mathscr{L}_{w, w}=\frac{1}{R e}\left(\frac{\partial^{2}}{\partial x^{2}}+\frac{\partial^{2}}{\partial y^{2}}-\beta^{2}\right)-\bar{u} \frac{\partial}{\partial x}-\bar{v} \frac{\partial}{\partial y}-i \beta \bar{w} \\ \mathscr{L}_{w, p}=-i \beta \\ \mathscr{R}_{w, w}=-i\end{array}\right.$

$\left\{\begin{array}{l}\mathscr{L}_{p, u}=\frac{\partial}{\partial x} \\ \mathscr{L}_{p, v}=\frac{\partial}{\partial v} \\ \mathscr{L}_{p, w}=i \beta\end{array}\right.$ 


\section{APÊNDICE B - Operador linear}

\section{compressível}

$$
\mathscr{L}=\left[\begin{array}{ccccc}
\mathscr{L}_{u, u} & \mathscr{L}_{u, v} & \mathscr{L}_{u, w} & \mathscr{L}_{u, p} & \mathscr{L}_{u, T} \\
\mathscr{L}_{v, u} & \mathscr{L}_{v, v} & \mathscr{L}_{v, w} & \mathscr{L}_{v, p} & \mathscr{L}_{v, T} \\
\mathscr{L}_{w, u} & \mathscr{L}_{w, v} & \mathscr{L}_{w, w} & \mathscr{L}_{w, p} & \mathscr{L}_{w, T} \\
\mathscr{L}_{p, u} & \mathscr{L}_{p, v} & \mathscr{L}_{p, w} & \mathscr{L}_{p, p} & \mathscr{L}_{p, T} \\
\mathscr{L}_{T, u} & \mathscr{L}_{T, v} & \mathscr{L}_{T, w} & \mathscr{L}_{T, p} & \mathscr{L}_{T, T}
\end{array}\right]
$$

$$
\mathscr{R}=\left[\begin{array}{ccccc}
\mathscr{R}_{u, u} & 0 & 0 & 0 & 0 \\
0 & \mathscr{R}_{v, v} & 0 & 0 & 0 \\
0 & 0 & \mathscr{R}_{w, w} & 0 & 0 \\
0 & 0 & 0 & \mathscr{R}_{p, p} & \mathscr{R}_{p, T} \\
0 & 0 & 0 & 0 & \mathscr{R}_{T, T}
\end{array}\right]
$$




$$
\begin{aligned}
& \int \mathscr{L}_{u, u}=-\frac{4}{3} \frac{\bar{\mu}}{R e} \partial_{x x}^{2}+\left[-\frac{4}{3} \frac{1}{R e}\left(\frac{d \bar{\mu}}{d \bar{T}}\right) \bar{T}_{x}+\bar{\rho} \bar{u}\right] \partial_{x}-\frac{\bar{\mu}}{R e} \partial_{y y}^{2}+\left[-\frac{1}{R e}\left(\frac{d \bar{\mu}}{d \bar{T}}\right) \bar{T}_{y}+\bar{\rho} \bar{v}\right] \partial_{y} \\
& +\frac{\beta^{2} \bar{\mu}}{R e}+2 \bar{\rho} \bar{u}_{x}+\bar{\rho} \bar{v}_{y}+i \beta \bar{\rho} \bar{w}+\bar{u} \bar{\rho}_{x}+\bar{v} \bar{\rho}_{y} \\
& \mathscr{L}_{u, v}=-\frac{1}{3} \frac{\bar{\mu}}{R e} \partial_{x y}^{2}-\frac{1}{R e}\left(\frac{d \bar{\mu}}{d \bar{T}}\right) \bar{T}_{y} \partial_{x}+\frac{2}{3} \frac{1}{R e}\left(\frac{d \bar{\mu}}{d \bar{T}}\right) \bar{T}_{x} \partial_{y}+\bar{\rho} \bar{u}_{y} \\
& \mathscr{L}_{u, w}=-\frac{i}{3} \frac{\beta}{R e} \bar{\mu} \partial_{x}+\frac{2}{3} i \frac{\beta}{R e}\left(\frac{d \bar{\mu}}{d \bar{T}}\right) \bar{T}_{x} \\
& \mathscr{L}_{u, p}=\frac{1}{\gamma M^{2}} \partial_{x}+\frac{1}{\bar{T}}\left(\bar{u} \bar{u}_{x}+\bar{v} \bar{u}_{y}\right) \\
& \mathscr{L}_{u, T}=-\frac{1}{R e}\left(\frac{d \bar{\mu}}{d \bar{T}}\right)\left(\frac{4}{3} \bar{u}_{x}-\frac{2}{3} \bar{v}_{y}\right) \partial_{x}-\frac{1}{R e}\left(\frac{d \bar{\mu}}{d \bar{T}}\right)\left(\bar{u}_{y}+\bar{v}_{x}\right) \partial_{y} \\
& -\frac{1}{R e}\left(\frac{d \bar{\mu}}{d \bar{T}}\right)\left(i \beta \bar{w}_{x}+\frac{4}{3} \bar{u}_{x x}+\bar{u}_{y y}+\frac{1}{3} \bar{v}_{x y}\right) \\
& -\frac{1}{R e}\left(\frac{d^{2} \bar{\mu}}{d \bar{T}^{2}}\right)\left(\frac{4}{3} \bar{T}_{x} \bar{u}_{x}+\bar{T}_{y} \bar{u}_{y}+\bar{T}_{y} \bar{v}_{x}-\frac{2}{3} \bar{T}_{x} \bar{v}_{y}\right) \\
& -\frac{\bar{\rho}}{\bar{T}}\left(\bar{u} \bar{u}_{x}+\bar{v} \bar{u}_{y}\right) \\
& \mathscr{R}_{u, u}=i \bar{\rho}
\end{aligned}
$$

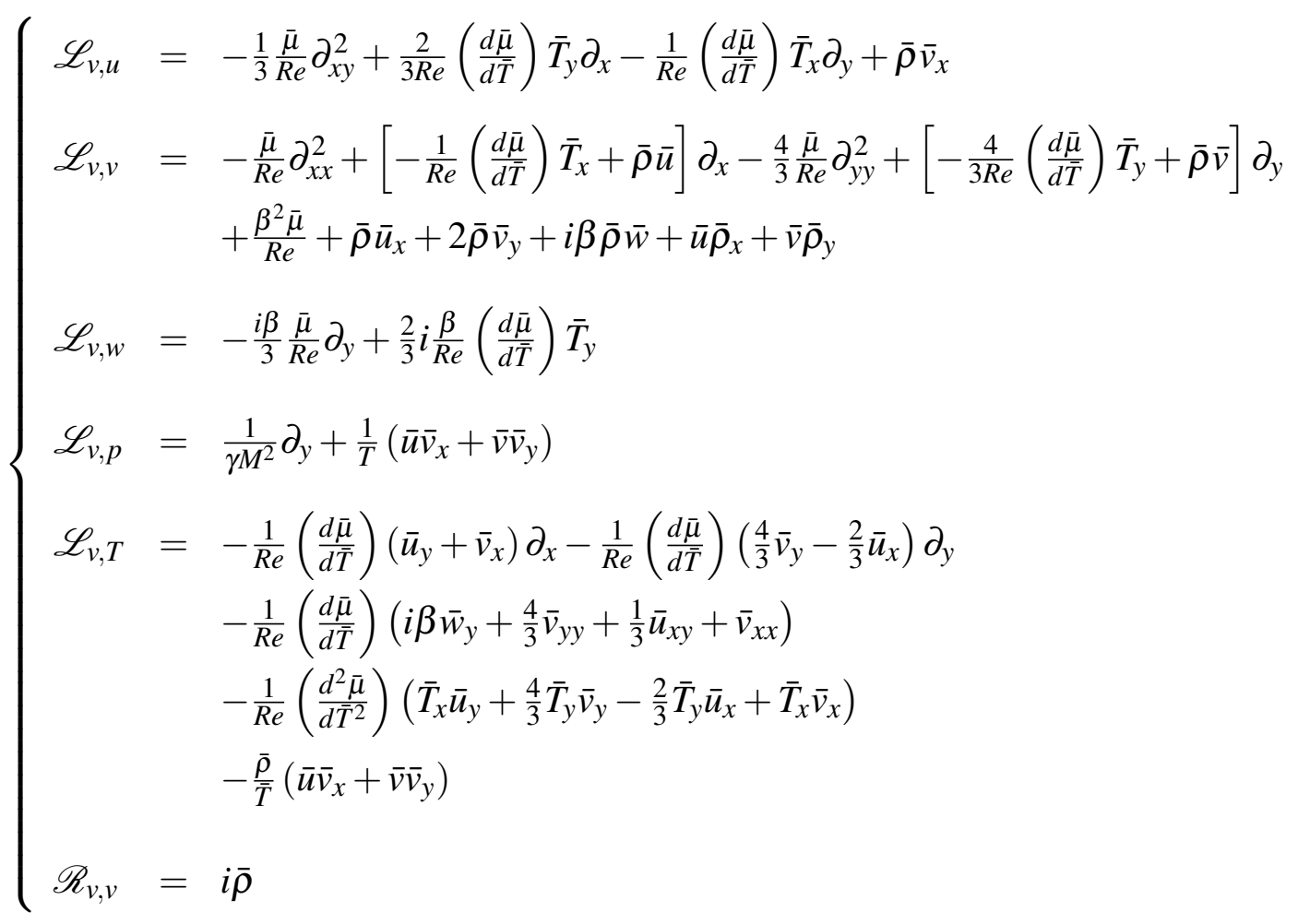




$$
\left\{\begin{aligned}
\mathscr{L}_{w, u}= & -\frac{i \beta}{3} \frac{\bar{\mu}}{R e} \partial_{x}-\frac{i \beta}{R e}\left(\frac{d \bar{\mu}}{d \bar{T}}\right) \bar{T}_{x}+\bar{\rho} \bar{w}_{x} \\
\mathscr{L}_{w, v}= & -\frac{i \beta}{3} \frac{\bar{\mu}}{R e} \partial_{y}-\frac{i \beta}{R e}\left(\frac{d \bar{\mu}}{d \bar{T}}\right) \bar{T}_{y}+\bar{\rho} \bar{w}_{y} \\
\mathscr{L}_{w, w}= & -\frac{\bar{\mu}}{\operatorname{Re}} \partial_{x x}^{2}+-\frac{\bar{\mu}}{R e} \partial_{y y}^{2}+\left[-\frac{1}{R e}\left(\frac{d \bar{\mu}}{d \bar{T}}\right)\left(\bar{u}_{y}+\bar{v}_{x}\right)\right] \partial_{x}+\left[-\frac{1}{R e}\left(\frac{d \bar{\mu}}{d \bar{T}}\right) \bar{T}_{y}+\bar{\rho} \bar{v}\right] \partial_{y} \\
& +\frac{4 \bar{\mu}}{3 R e} \beta^{2}+\bar{\rho} \bar{u}_{x}+\bar{\rho} \bar{v}_{y}+i \beta \bar{\rho} \bar{w}+\bar{u} \bar{\rho}_{x}+\bar{v} \bar{\rho}_{y} \\
\mathscr{L}_{w, p}= & \frac{i \beta}{\gamma M^{2}}+\frac{1}{\bar{T}}\left(\bar{u}_{x}+\bar{v}_{x}\right) \\
\mathscr{L}_{w, T}= & -\frac{1}{R e}\left(\frac{d \bar{\mu}}{d \bar{T}}\right) \bar{w}_{x} \partial_{x}-\frac{1}{R e}\left(\frac{d \bar{\mu}}{d \bar{T}}\right) \bar{w}_{y} \partial_{y}-\frac{1}{R e}\left(\frac{d \bar{\mu}}{d \bar{T}}\right)\left[\bar{w}_{x x}+\bar{w}_{y y}-\frac{2}{3} i \beta\left(\bar{u}_{x}+\bar{v}_{y}\right)\right] \\
& -\frac{1}{R e}\left(\frac{d^{2} \bar{\mu}}{d \bar{T}^{2}}\right)\left[\bar{T}_{x} \bar{w}_{x}+\bar{T}_{y} \bar{w}_{y}\right]-\bar{\rho}_{\bar{T}}\left(\bar{u} \bar{w}_{x}+\bar{v} \bar{w}_{y}\right) \\
\mathscr{R}_{w, w}= & i \bar{\rho}
\end{aligned}\right.
$$

$$
\left\{\begin{array}{l}
\mathscr{L}_{p, u}=\bar{\rho} \bar{T} \partial_{x}+\bar{T} \bar{\rho}_{x} \\
\mathscr{L}_{p, v}=\bar{\rho} \bar{T} \partial_{y}+\bar{T} \bar{\rho}_{y} \\
\mathscr{L}_{p, w}=i \beta \bar{\rho} \bar{T} \\
\mathscr{L}_{p, p}=\bar{u} \partial_{x}+\bar{v} \partial_{y}+i \beta \bar{w}+\bar{u}_{x}+\bar{v}_{y}-\frac{1}{\bar{T}}\left(\bar{u} \bar{T}_{x}+\bar{v} \bar{T}_{y}\right) \\
\mathscr{L}_{p, T}=-\bar{\rho} \bar{u} \partial_{x}-\bar{\rho} \bar{v} \partial_{y}-\bar{\rho} \bar{u}_{x}-\bar{\rho} \bar{v}_{y}-i \beta \bar{\rho} \bar{w}-\bar{u} \bar{\rho}_{x}-\bar{v} \bar{\rho}_{y}+\frac{\bar{\rho}}{\bar{T}}\left(\bar{u} \bar{T}_{x}+\bar{v} \bar{T}_{y}\right) \\
\mathscr{R}_{p, p}=i \\
\mathscr{R}_{p, T}=-i \bar{\rho}
\end{array}\right.
$$




$$
\begin{aligned}
& \int \mathscr{L}_{T, u}=\left[-\frac{2 \gamma(\gamma-1) \bar{\mu} M^{2}}{\operatorname{Re}}\left(\frac{4}{3} \bar{u}_{x}-\frac{2}{3} \bar{v}_{y}\right)+\gamma \bar{\rho} \bar{T}\right] \partial_{x}+\left[-\frac{2 \gamma(\gamma-1) \bar{\mu} M^{2}}{\operatorname{Re}}\left(\bar{u}_{y}+\bar{v}_{x}\right)\right] \partial_{y} \\
& -2 \gamma(\gamma-1) \frac{i \beta \bar{\mu} M^{2}}{R e} \bar{w}_{x}+\bar{T} \bar{\rho}_{x}+\bar{\rho} \bar{T}_{x} \\
& \mathscr{L}_{T, v}=\left[-\frac{2 \gamma(\gamma-1) \bar{\mu} M^{2}}{\operatorname{Re}}\left(\bar{u}_{y}+\bar{v}_{x}\right)\right] \partial_{x}+\left[-\frac{2 \gamma(\gamma-1) \bar{\mu} M^{2}}{\operatorname{Re}}\left(-\frac{2}{3} \bar{u}_{x}+\frac{4}{3} \bar{v}_{y}\right)+\gamma \bar{\rho} \bar{T}\right] \partial_{y} \\
& -2 \gamma(\gamma-1) \frac{i \beta \bar{\mu} M^{2}}{R e} \bar{w}_{y}+\bar{T} \bar{\rho}_{y}+\bar{\rho} \bar{T}_{y} \\
& \mathscr{L}_{T, \hat{w}}=\left[-\frac{2 \gamma(\gamma-1) \bar{\mu} M^{2}}{R e} \bar{w}_{x}\right] \partial_{x}+\left[-\frac{2 \gamma(\gamma-1) \bar{\mu} M^{2}}{R e} \bar{w}_{y}\right] \partial_{y} \\
& +\frac{4}{3} \gamma(\gamma-1) \frac{i \bar{\mu} \beta M^{2}}{R e}\left(\bar{u}_{x}+\bar{v}_{y}\right)+i \beta \gamma \bar{\rho} \bar{T} \\
& \mathscr{L}_{T, p}=\bar{u} \partial_{x}+\bar{v} \partial_{y}+i \beta \bar{w}+\gamma\left(\bar{u}_{x}+\bar{v}_{y}\right) \\
& \mathscr{L}_{T, T}=-\frac{\gamma \bar{\kappa}}{\operatorname{RePr}} \partial_{x x}^{2}+\left[-2 \frac{\gamma}{\operatorname{RePr}}\left(\frac{d \bar{\kappa}}{d \bar{T}}\right) \bar{T}_{x}\right] \partial_{x}-\frac{\gamma \bar{\kappa}}{\operatorname{RePr}} \partial_{y y}^{2}+\left[-2 \frac{\gamma}{\operatorname{RePr}}\left(\frac{d \bar{\kappa}}{d \bar{T}}\right) \bar{T}_{y}\right] \partial_{y} \\
& +\frac{\beta^{2} \gamma \bar{\kappa}}{\operatorname{RePr}}-\frac{\gamma}{\operatorname{RePr}}\left(\frac{d \bar{\kappa}}{d \bar{T}}\right)\left(\bar{T}_{x x}+\bar{T}_{y y}\right)-\frac{\gamma}{\operatorname{RePr}}\left(\frac{d^{2} \bar{\kappa}}{d \bar{T}^{2}}\right)\left(\bar{T}_{x}^{2}+\bar{T}_{y}^{2}\right) \\
& -\frac{\gamma(\gamma-1) M^{2}}{\operatorname{Re}}\left(\frac{d \bar{\mu}}{d \bar{T}}\right)\left(\frac{4}{3} \bar{u}_{x}^{2}+\bar{v}_{x}^{2}+\bar{w}_{x}^{2}+\bar{u}_{y}^{2}+\frac{4}{3} \bar{v}_{y}^{2}+\bar{w}_{y}^{2}-\frac{4}{3} \bar{u}_{x} \bar{v}_{y}+2 \bar{u}_{y} \bar{v}_{x}\right) \\
& \mathscr{R}_{T, p}=i
\end{aligned}
$$

